Portland State University

PDXScholar

6-2-2021

\title{
Investigations into Size and Surface Control of Silicon Nanocrystals for Improved Optical Properties
}

James Donald Barnes

Portland State University

Follow this and additional works at: https://pdxscholar.library.pdx.edu/open_access_etds

Part of the Chemistry Commons, and the Nanoscience and Nanotechnology Commons Let us know how access to this document benefits you.

\section{Recommended Citation}

Barnes, James Donald, "Investigations into Size and Surface Control of Silicon Nanocrystals for Improved Optical Properties" (2021). Dissertations and Theses. Paper 5710.

https://doi.org/10.15760/etd.7582

This Dissertation is brought to you for free and open access. It has been accepted for inclusion in Dissertations and Theses by an authorized administrator of PDXScholar. Please contact us if we can make this document more accessible: pdxscholar@pdx.edu. 


\title{
Investigations into Size and Surface Control of Silicon Nanocrystals for Improved Optical Properties
}

\author{
by \\ James Donald Barnes
}

\begin{abstract}
A dissertation submitted in partial fulfilment of the requirements for the degree of
\end{abstract}

\author{
Doctor of Philosophy \\ in \\ Chemistry
}
Dissertation Committee:
Andrea Goforth, Chair
Robert Strongin
David Stuart
Rolf Koenenkamp

Portland State University
2021 
Abstract

The discovery of visible photoluminescence $(\mathrm{PL})$ from nanocrystalline porous silicon in 1990 led to extensive research into the mechanisms of the emergent properties, and optimization of these properties, for use in applications. The widespread use of silicon nanoparticles (Si NPs) in commercial applications is currently limited by three main factors: 1) poor radiative recombination efficiency of the interband transition, 2) instability of the interband photoluminescence, and 3) a lack of scalable methods for producing Si NPs that are both highly crystalline and size monodisperse.

To address these limitations, this dissertation correlates changes in the photoluminescence properties of hydrogen passivated silicon nanoparticles $(\mathrm{H}-\mathrm{Si}$ NPs) with changes in the surface structure (Chapters 2 and 3 ), as well as develops new synthesis methodology to produce larger, more crystalline Si NPs (Chapter $4)$.

In Chapters 2 and 3, red photoluminescent $\mathrm{H}$-Si NPs were prepared by high temperature reductive annealing of a $\left[\mathrm{HSiO}_{1.5}\right]_{\mathrm{n}}$ polymer derived from $\mathrm{HSiCl}_{3}$, followed by an aqueous HF etching procedure to isolate them in colloidal form. The H-Si NPs were then subjected to different chemical and physical environments and the changes to the photoluminescence spectra were then related to the changes seen in other spectroscopic measurements. 
First (Chapter 2), the stability of the interband transition of H-Si NPs was probed using the free radical (2,2,6,6-Tetramethylpiperidin-1-yl)oxyl (TEMPO) under different lighting conditions (dark, ambient, and UV) and the photoluminescence spectra of these samples were monitored over time. The TEMPO radical was observed to increase the interband emission intensity, but with a large hypochromic shift that is correlated to the significant oxidation of the Si NP surface. We propose that this shift is due to core shrinkage of the Si NP upon oxidation, and not an emergent electronically active defect state from the resultant surface oxidation.

Second (Chapter 3), a surface treatment for Si NPs was developed to stabilize the interband transition using purposeful oxidation. A survey of chemical environments that have been shown in the literature to promote oxidation of $\mathrm{Si}$ (ethanol, acetone, DMSO, and benzoyl peroxide (BPO)) was conducted, and based on spectroscopic results, BPO was identified as a reagent that can be used to oxidize the surface of the H-Si NPs without causing a significant hypochromic shift in the interband transition. It was observed that the surface reaction in the presence of BPO was accelerated by continuous $365 \mathrm{~nm}$ irradiation, resulting in an increase in interband transition intensity with no shift in the emission energy.

Finally (Chapter 4), the application of metallothermic reduction of silicon oxides was probed as a potential alternative to the high temperature reductive annealing synthesis method, with the aim of achieving higher crystallinity Si NPs with similar or better size polydispersity. Initially, a $\left[\mathrm{HSiO}_{1.5}\right]_{\mathrm{n}}$ polymer was reduced 
by $\mathrm{Mg}$ powder to produce highly crystalline, diamond lattice $\mathrm{Si}^{0}$ with nano-sized crystalline domains that either fused into larger structures or maintained original particle morphology depending on processing conditions. Attempting to control the processing conditions to leverage the latter result, nanoscale $\mathrm{SiO}_{2}$ template particles of known size and morphology were next metallothermically reduced to try to produce replica Si NPs of equal size and shape. Although some conditions were modestly successful in terms of producing crystalline Si NPs with morphology retention of the template, most synthetic trials only produced fused micron-sized structures of crystalline $\mathrm{Si}$. It is possible that with better spatial control over local heats of reaction, that individual $\mathrm{SiO}_{2}$ nanoparticles can be reduced to individual Si NPs without the fusion of neighboring domains. 


\section{Acknowledgements}

There are many people that helped me throughout this journey, and I would like to take a moment to thank them.

I would like to thank my graduate advisor and dissertation committee chair Dr. Andrea Goforth for her unwavering faith in my abilities. Without her support, both academic and emotional, I would not have been able to accomplish this goal. I would also like to thank the rest of my dissertation committee, Dr. Robert Strongin, Dr. David Stuart, and Dr. Rolf Koenenkamp. This work would be considerably lessened without their academic guidance and contributions.

To my friends and family, I thank you for your patience and support. This has been a long process and you all never stopped believing in me. I hope that I will now have more time to help in the garden, get a round of golf in, catch some playoff hockey games on the tube, and spend an afternoon at a soccer game.

Finally, to my partner in life Ruth. Without you this would not have been possible. I have not always made things easy, but your love and patience has gotten me through some of the darkest times. Now that this adventure is coming to an end, I look forward to the many new adventures to come with you. 
Abstract.

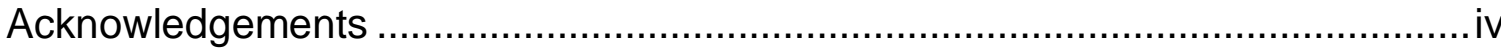

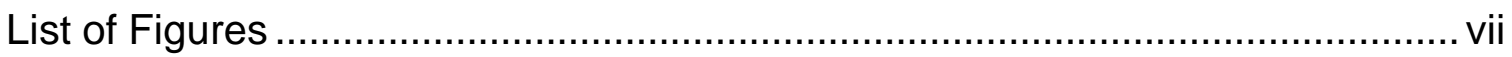

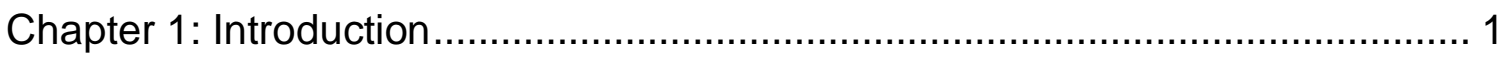

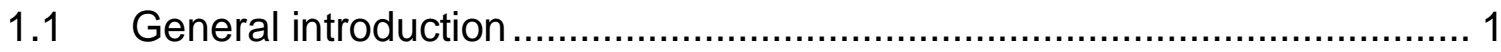

$1.2 \quad$ Electronic structure of Si NPs ........................................................ 3

1.3 Effects of defects and surface states on the photoluminescence of Si NPs

1.4 Our synthetic method and the development of crystalline defects

1.5 Motivation for the research presented herein ........................................ 10

Chapter 2: Effects of (2,2,6,6-Tetramethylpiperidin-1-yl)oxyl (TEMPO) and lighting conditions on the surface composition of hydride terminated silicon

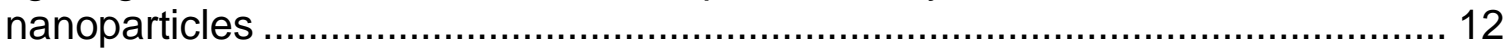

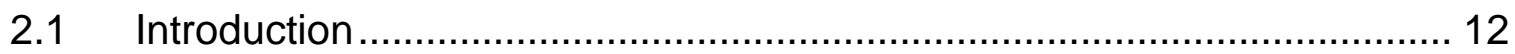

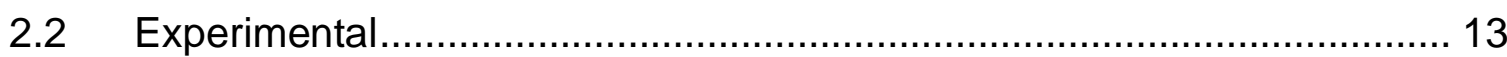

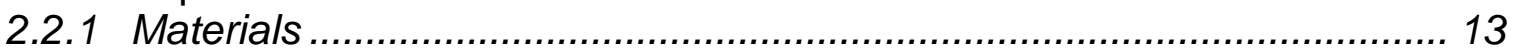

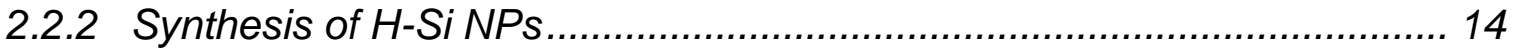

2.2.3 TEMPO surface treatment ................................................................... 15

2.2.4 Photoluminescence measurements and analysis ................................ 16

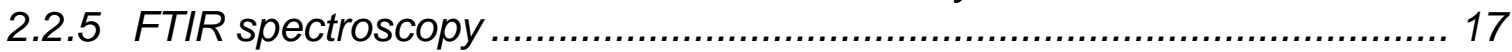

2.2.6 X-ray photoelectron spectroscopy (XPS) measurements....................... 18

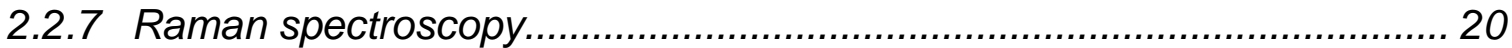

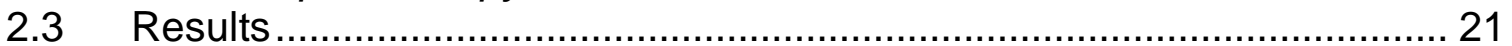

2.3.1 H-Si NP PL spectra as a function of TEMPO concentration and lighting

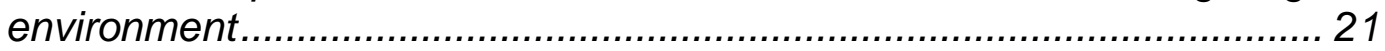

2.3.2 FTIR measurements before and after TEMPO treatment....................... 31

2.3.3 XPS measurements before and after treatment..................................... 36

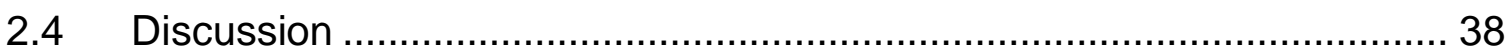

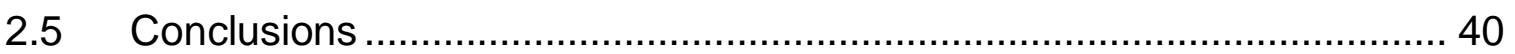

Chapter 3: Benzoyl peroxide as a surface passivating agent to improve photoluminescence intensity and colloidal stability of silicon nanoparticles........ 42

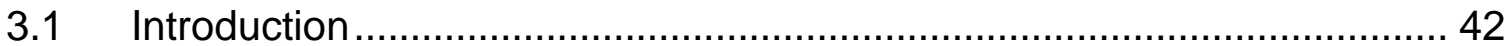

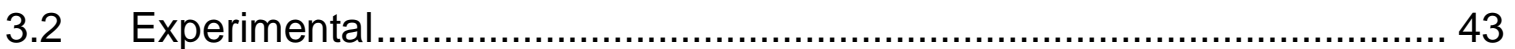

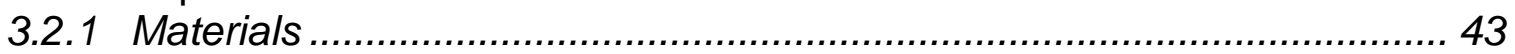

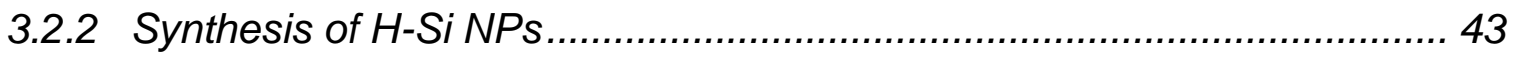




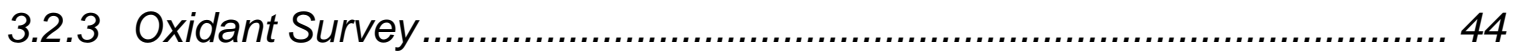

3.2.4 Benzoyl peroxide surface treatment study ......................................... 45

3.2.5 Photoluminescence measurements.................................................. 45

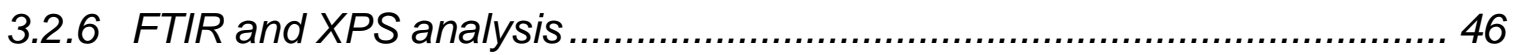

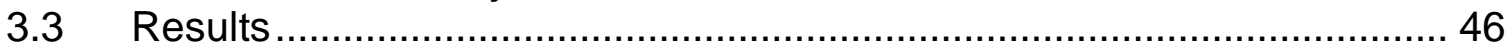

3.3.1 Oxidant Survey ........................................................................... 46

3.3.2 PL measurements from benzoyl peroxide treatment ............................ 47

3.3.3 FTIR measurements before and after BPO treatment ........................... 52

3.3.4 XPS measurements before and after treatment...................................5 56

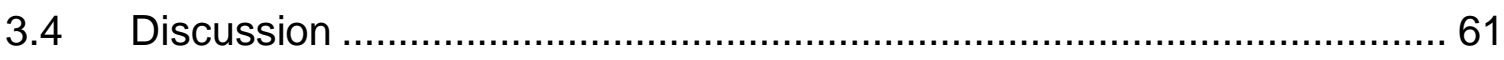

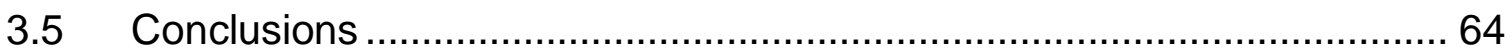

Chapter 4: Investigation into magnesiothermic reduction of silicon oxides as a synthetic route to free-standing nanocrystalline silicon..................................... 65

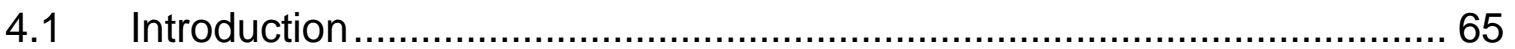

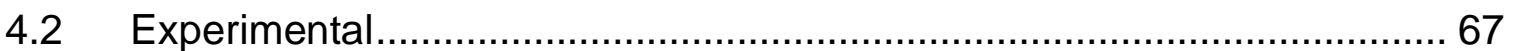

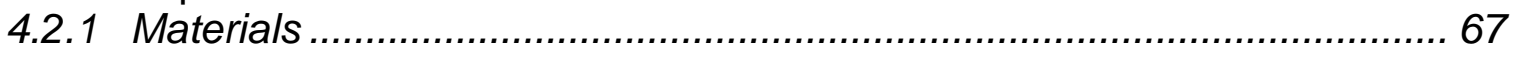

4.2.2 Nanocrystalline Silicon from magnesiothermic reduction ....................... 67

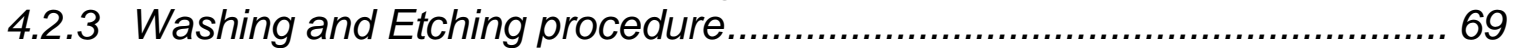

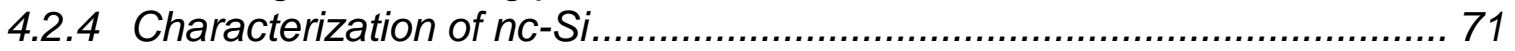

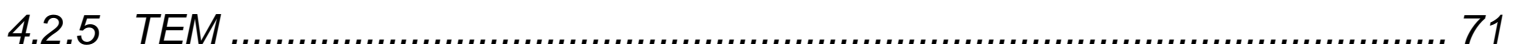

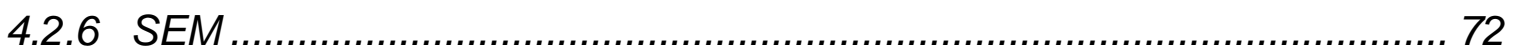

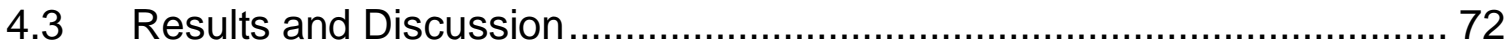

4.3.1 Preliminary reduction of $\left[\mathrm{HSiO}_{1.5}\right]_{n}$ with magnesium metal...................... 72

4.3.2 Effects of time, temperature, and reactant concentrations on product

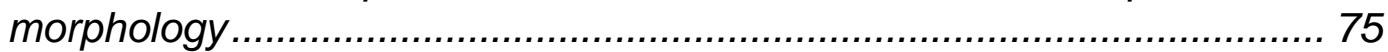

4.3.3 Addition of heat sink to reduce crystallite fusion .................................... 81

4.3.4 Magnesiothermic reduction of $\mathrm{SiO}_{2}$ spheres ....................................... 83

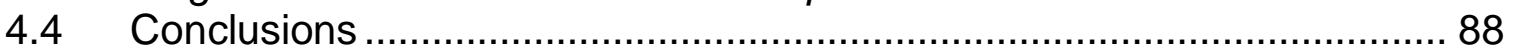

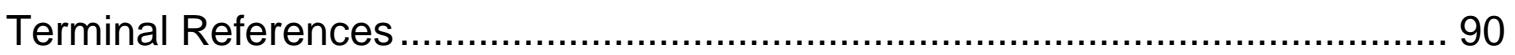




\section{List of Figures}

Figure 1.1: Si NPs at different stages of processing ………......................... 9

Figure 2.1: Binding energy in the $\mathrm{Si}(2 \mathrm{p})$ region for $\mathrm{H}$-Si NPs before treatment. 19

Figure 2.2: PL spectra for the control sample and fit plot analysis of one time point.

Figure 2.3: PL intensity and lambda max for the control samples..................... 25

Figure 2.4: PL intensity and lambda max for the $1 \mathrm{mM}$ TEMPO samples. ........ 27

Figure 2.5: PL intensity and lambda max for the $5 \mathrm{mM}$ TEMPO samples. ........ 29

Figure 2.6: FTIR spectra for set of control samples (no TEMPO)..................... 32

Figure 2.7: FTIR spectra for set of 1 mM TEMPO treated samples.................. 34

Figure 2.8: FTIR spectra for set of 5 mM TEMPO treated samples.................. 35

Figure 2.9: XPS binding energies in the $\mathrm{Si}(2 \mathrm{p})$ region before and after treatments for the control sample (a) and for the $5 \mathrm{mM}$ TEMPO treatment (b).... 36

Figure 3.1: H-Si NPs in toluene after 4 days of exposure to the chemical oxidants under $365 \mathrm{~nm}$ irradiation.

Figure 3.2: PL spectra (excited at $365 \mathrm{~nm}$ ) of $\mathrm{H}$-Si NPs in toluene with variable concentration of BPO, taken after 24 hours of continuous irradiation of the samples with $365 \mathrm{~nm}$ light.

Figure 3.3: PL spectra (excited at $365 \mathrm{~nm}$ ) of $\mathrm{H}$-Si NPs in toluene with variable concentration of BPO, taken after 24 days of treatment in the dark.

Figure 3.4: PL measurements before and after washing dark BPO treatment to remove blue emissive component. ............................................................ 50

Figure 3.5: FTIR of BPO and UV treated samples........................................ 52

Figure 3.6: FTIR of BPO treated samples in the dark ..................................... 54

Figure 3.7: XPS spectra of the BPO and UV treated Si NPs........................... 56

Figure 3.8: XPS spectra of the BPO treated Si NPs held in the dark................ 58

Figure 3.9: XPS of starting material showing the modeling procedure used. .... 59 
Figure 3.10: Percentage of intensity vs BPO concentration for each oxidation state 60

Figure 4.1: XRD patterns and TEM images from $\mathrm{Mg}$ reduction of $\left[\mathrm{HSiO}_{1.5}\right]_{\mathrm{n}} \ldots . .73$

Figure 4.2: XRD patterns of the annealed product mixture with varying reactant ratios. 76

Figure 4.3: XRD patterns of the annealed product mixture with varying reaction temperature.

Figure 4.4: TEM images of $\mathrm{H}$-terminated $\mathrm{nc}-\mathrm{Si}$ annealed with and without $\mathrm{NaCl}$. 82

Figure 4.5: TEM images of $\mathrm{SiO}_{2}$ and $\mathrm{Si}$ NPs before and after reduction. 84

Figure 4.6: SEM images of $\mathrm{SiO}_{2}$ before and nc-Si after reduction. .................... 86

Figure 4.7: XRD patterns after the reduction of three $\mathrm{SiO}_{2}$ precursors. 87 


\section{Chapter 1: Introduction}

\subsection{General introduction}

Since the first report of visible photoluminescence from nanocrystalline silicon (nc-Si) by Canham in $1990,{ }^{1}$ silicon nanoparticles (Si NPs) have been a popular area of research for their potential use in optoelectronic applications. With the lower expected toxicity of silicon nanoparticles, as compared to popular heavy metal-based quantum dot (QD) light emitters, such as CdSe QDs, Si NPs represent a welcome alternative photoactive material. However, there are numerous challenges that have prevented $\mathrm{Si}$ NPs from widespread use. Photoluminescent Si NPs have been synthesized, and are being explored as functional materials in numerous optical applications, including chemical sensing ${ }^{2-5}$ biological imaging ${ }^{6-8}$ and optical-electronic devices ${ }^{9-11}$ All of these applications require control over particle size and crystallinity, as well as surface structure, to realize the desired set of photophysical properties in each case.

As one example, photoluminescent Si NPs have been shown to be effective at sensing a variety of chemicals, such as nitro-functionalized compounds and formaldehyde, via photo-induced electron transfer that causes Si NP intensity quenching; ${ }^{2-5}$ such sensor-analyte interactions are dependent on size-related electronic transitions in the Si NPs. As another example, in biological imaging applications, red to near-IR emission is desired due to the low absorption of these wavelengths by body tissues, which allows visualization of the imaging agent. 
There are numerous protocols reported for producing Si NPs with red photoluminescence, ${ }^{12-14}$ but the stability of the emission intensity and wavelength over time in oxidative environments is still poor; further, the low crystallinity of these particles may limit the attainable photoluminescence quantum yield. ${ }^{15-17}$

Finally, optical-electronic applications currently use direct band gap II-VI and III-V nanoparticle semiconductors as the optical components because of their size dependent optical output (i.e., wavelength), high absorptivity, and high photoluminescence quantum yields. However, due to the integration required with the silicon based electronics in modern devices, a silicon-based optical component would be more desirable. ${ }^{18}$ Currently, commercial integration of Si NPs in optoelectronic devices is limited by the low efficiency of radiative recombination, as well as instability of the photoluminescence intensity and emission wavelength over time. ${ }^{15,16,19,20}$ In all of these applications, synthetic control over the size, crystallinity, and surface chemical termination of the Si NPs must be realized in order to optimize the intrinsic absorption and emission properties of the material.

This dissertation will address two of the main limitations that are preventing widespread use of Si NPs for their optical properties in devices. First, by studying the effects of the chemical and physical environment on the optical properties we will demonstrate stabilization of the optical properties of Si NP suspensions. Second, we will also explore a new synthetic route to increase control over particle size and crystallinity that is currently limited. 


\subsection{Electronic structure of Si NPs}

Semiconductors can be separated into two categories: direct band gap semiconductors such as CdSe and indirect band gap semiconductors such as $\mathrm{Si}$. Direct band gap semiconductors have the maxima of the valence band aligned in momentum space with the minima of the conduction band while indirect band gap semiconductors do not have the maxima and minima aligned in momentum space. Due to momentum conservation $(\Delta \mathrm{k}=0)$ rules for allowed electronic transitions, direct band gap semiconductors $(\Delta \mathrm{k}=0)$ will allow radiative recombination across the band gap while indirect band gap semiconductors $(\Delta k \neq 0)$ will not allow recombination without the assistance of a phonon (lattice vibration).

As crystalline semiconductors are reduced in size, such that they approach the Bohr exciton radius, the electronic properties of the material are expected to change. ${ }^{21,22}$ Once the Bohr exciton radius is reached the band gap of the material is expected to increase in energy with further reductions in the diameter of the particle. This size dependent band gap energy is due to the removal of states at or near the band edges from the removal of atoms, and thus atomic orbitals, from the nanocrystal surface.

The band gap for $\mathrm{Si}$ is $1.1 \mathrm{eV}$ as a bulk crystal, however once the nanocrystal size has been reduced to below $5 \mathrm{~nm}$ in diameter, visible photoluminescence has been observed. The size dependence of the 
photoluminescence has previously been proposed by Trwoga ${ }^{23}$ and experimentally demonstrated by Korgel ${ }^{12}$ and follows the effective mass approximation (eq 1.1 below).

$$
E(d)=E_{g}+\frac{h^{2} \pi^{2}}{2 d^{2}}\left(\frac{1}{m_{e}^{*}}+\frac{1}{m_{h}^{*}}\right)-\frac{1.786 e^{2}}{\varepsilon_{r} d}
$$

In equation 1.1, $d$ is the nanoparticle diameter, $E(d)$ is the quantum confined band gap energy of the Si NP, $E_{g}$ is the bulk band gap energy of $\mathrm{Si}(1.1 \mathrm{eV})$, e is the electron charge, $m_{e}\left(0.19 m_{0}\right)$ and $m_{h}\left(0.286 m_{0}\right)$ are the effective electron and hole masses, and $\varepsilon_{r}$ is the relative permittivity of silicon (11.86).

The indirect nature of the band gap in bulk Si results in phonon assisted recombination across the band gap. As the nanocrystal decreases in size the wave functions of the hole and excited electron spread in momentum space, thus resulting in some overlap in their respective wavefunctions. ${ }^{24}$ This overlap allows for an increased probability of radiative recombination and the overlap continues to increase as the nanocrystal size is further reduced and the oscillator strength increases. ${ }^{23-25}$

1.3 Effects of defects and surface states on the photoluminescence of Si NPs Due to the large number of Si atoms at the surface in a typical Si NP, there are numerous $\mathrm{Si}$ atoms that lack complete coordination (4 bonds) with other $\mathrm{Si}$ atoms. As a result, high energy surface defects like free radicals (dangling bonds) and $\mathrm{Si}=\mathrm{Si}$ dimers will fill the remaining coordination sites. ${ }^{26,27}$ These defect states 
are unstable and will react readily to passivating agents such as molecular or atomic hydrogen.

Hydrogen passivation has been used in computational studies of Si NPs due to the minimal effect it has on the electronic structure of the Si NP. ${ }^{20,28,29}$ Hydride passivation has also been previously demonstrated experimentally to result in photoluminescence that is entirely due to the interband transition (the quantum confined core). ${ }^{12,30,31}$ Although the $\mathrm{Si}-\mathrm{H}$ surface passivation is considered ideal for studies of the interband transitions of Si NPs, the Si-H bond is relatively unstable when compared to more polar $\mathrm{Si}-\mathrm{X}$ bonds where $\mathrm{X}$ is a more electronegative atom $(\mathrm{O}, \mathrm{N}, \mathrm{C}, \mathrm{Cl}$, etc.) and would be expected to readily form defects in environmental and experimental conditions.

Surface states of QDs have also been shown to affect the maximum photoluminescence $(\mathrm{PL})$ wavelength as well as PL intensity, depending on the type of surface defects. ${ }^{27-30}$ It is well known that dangling bonds (radicals) at the nanoparticle surface can provide exciton trap states that result in non-radiative recombination, ${ }^{28}$ lowering the quantum yield of the Si NPs. Other chemical surface states can result in radiative recombination centers that have energies sitting in the middle in the band gap can dominate the emission of the NPs due to the long excited state lifetime for interband recombination in Si NPs. ${ }^{32}$ Due to the reactive nature of the $\mathrm{Si-H}$ surface further passivation is generally performed as a protectant for use in applications. 
Alkyl-passivation is generally regarded as the most effective way to preserve interband recombination without introducing electronically active states mid band gap. ${ }^{12,33}$ Numerous methods have been developed for creating Si-C bonds at the surface which are much more stable than the Si-H bonds they replace. The majority of these methods focus on a hydrosilylation reaction where $\mathrm{Si}-\mathrm{H}$ addition across an unsaturated hydrocarbon, typically a terminal olefin. The initial activation of the Si-H bond can be achieved either under UV light, ${ }^{34,35}$ at high temperatures, ${ }^{36-39}$ using a Lewis acid,$^{33}$ or radical initiators. ${ }^{40}$ Of these methods the thermal hydrosilylation provides the most surface coverage of up to $70 \%{ }^{37}$

The ideal surface passivation may in fact be an amorphous $\mathrm{SiO}_{2}$ layer. Due to the large band mismatch between the semiconducting Si NP and the insulating $\mathrm{SiO}_{2}$ coating there are theoretically no electronically active mid-band states. However, modeling studies of $\mathrm{Si} \mathrm{NPs}$ embedded in $\mathrm{SiO}_{2}$ have shown that at the interface there are distorted Si-Si bonds, bridging Si-O-Si bonds, and dangling bonds that all can provide trap states within the band gap. ${ }^{41-43}$

There are numerous literature reports on the effects of oxidation on the PL of $\mathrm{Si} \mathrm{NPs} \mathrm{that} \mathrm{are} \mathrm{not} \mathrm{embedded} \mathrm{in} \mathrm{an} \mathrm{SiO}_{2}$ matrix, however it is difficult to relate findings from one report to the next due to many seemingly contradictory reports. Some studies provide support for blue luminescence being the result of nitrogen related defects ${ }^{44}$ while others provide support for oxygen related defects being responsible. ${ }^{15}$ Other reports provide support for orange luminescence being the 
result of $\mathrm{Si}_{-} \mathrm{SiO}_{2}$ interfacial energy after oxide layer formation or from $\mathrm{Si}=\mathrm{O}$ bonding. ${ }^{19,20}$

1.4 Our synthetic method and the development of crystalline defects

The major routes to producing Si NPs are gas phase reduction of $\mathrm{SiH}_{4},{ }^{19,45}$ solution phase oxidation reactions of silicon polyanions (e.g., $\mathrm{Si}_{4}{ }^{4-}$ ) with conventional chemical oxidizing agents, ${ }^{46-49}$ laser ablation of bulk $\mathrm{Si}^{0}$ in solution, ${ }^{50,51}$ high temperature reductive annealing of silicon suboxide polymers ${ }^{14,52,53}$ followed by subsequent chemical etching, and solution phase reductions of silanes (e.g., $\mathrm{HSiCl}_{3}$ and others) with conventional chemical reducing agents. ${ }^{54,55}$ The method by which the Si NPs are synthesized has a very large impact on its optical properties due to the degree of crystallinity and degree of defects within the nanoparticle structure. Both laser ablation and gas phase pyrolysis of $\mathrm{SiH}_{4}$ produce particles that are very highly crystalline with size dependent optical properties. These methods are best used on a laboratory scale for observations of physical properties on ideal crystals, ${ }^{19,45,50,51}$ however the methods themselves are not viewed as scalable and thus do not hold much commercial promise. Solution phase methods are ideal for commercial scale up, however the Si NPs produced from these methods have a low crystallinity and do not show size dependent optical properties. ${ }^{46-49,54,55}$

Our group has developed a synthesis that has been shown previously to produce Si NPs via the high temperature reductive annealing of a silicon suboxide 
precursor. ${ }^{14} \mathrm{HSiCl}_{3}$ first undergoes a hydrolysis and polycondensation reaction with $\mathrm{H}_{2} \mathrm{O}$ to produce a $\left[\mathrm{HSiO}_{1.5}\right]_{n}$ polymer. The silicon suboxide polymer then thermally processed under reducing atmosphere at $1100^{\circ} \mathrm{C}$ for 10 hours to yield nc-Si . This process has been proposed to occur through three intermediate reactions as follows: ${ }^{2}$

$$
\begin{array}{ll}
4 \mathrm{HSiO}_{1.5}(\mathrm{~s}) \rightarrow 3 \mathrm{SiO}_{2}(\mathrm{~s})+\mathrm{SiH}_{4}(\mathrm{~g}) & 250^{\circ} \mathrm{C} \text { to } 350^{\circ} \mathrm{C} \\
\mathrm{SiH}_{4}(\mathrm{~g}) \rightarrow \mathrm{a}-\mathrm{Si}(\mathrm{s})+2 \mathrm{H}_{2}(\mathrm{~g}) & 350^{\circ} \mathrm{C} \text { to } 450^{\circ} \mathrm{C} \\
\mathrm{a}-\mathrm{Si}(\mathrm{s}) \rightarrow \mathrm{c}-\mathrm{Si}(\mathrm{s}) & 900^{\circ} \mathrm{C} \text { to } 1400^{\circ} \mathrm{C}
\end{array}
$$

With a temperature increase of $20^{\circ} \mathrm{C}$ per minute there is minimal loss of $\mathrm{SiH}_{4}$ but lower furnace ramp rates have shown significant loss of gaseous products. ${ }^{52}$ The composite powder that results can be removed from the furnace and ground to a fine powder in a mortar and pestle. The composite is light brown in color and highly photoluminescent under $365 \mathrm{~nm}$ irradiation (Figure $1.1 \mathrm{a}$ and $\mathrm{b}$ on next page). At higher processing temperatures, approaching the melting point of $\mathrm{Si}\left(1410^{\circ} \mathrm{C}\right)$, there is an increase in particle size from $3.1 \mathrm{~nm}$ at $1100^{\circ} \mathrm{C}$ up to $12.8 \mathrm{~nm}$ at $1350^{\circ} \mathrm{C}$ with a dwell time of 1 hour. ${ }^{12}$ Due to the temperature limit of $1100^{\circ} \mathrm{C}$ for our furnace, we are limited to Si NPs of approx. $3 \mathrm{~nm}$ to $4 \mathrm{~nm}$ with an increased dwell time of 10 hours. ${ }^{14}$

While high temperature reductive annealing can produce nc-Si with size dependent optical properties, the Si NPs embedded in the oxide matrix is not 


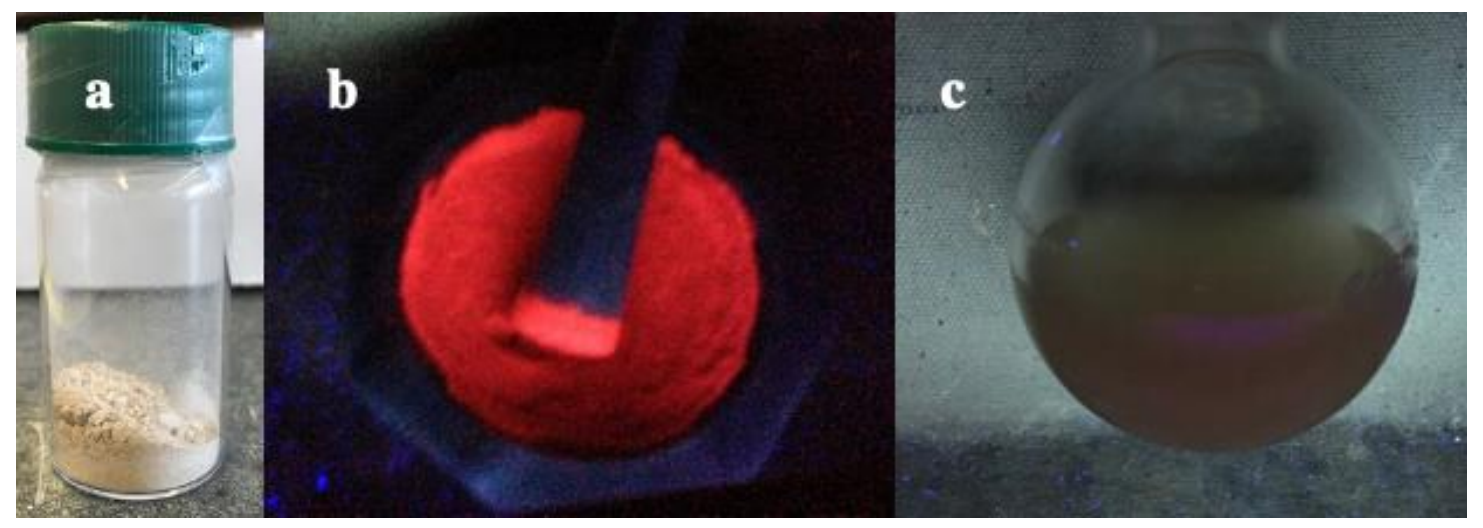

Figure 1.1: Si NPs at different stages of processing. (a) The composite material after reductive thermal processing, (b) composite material ground to a fine powder under $365 \mathrm{~nm}$ irradiation, and (c) H-Si NPs in toluene under $365 \mathrm{~nm}$ irradiation after aqueous HF etching procedure.

completely crystalline. Scanning tunneling microscopy (STS) and Raman microscopy was previously used on our H-Si NPs to verify the existence of amorphous Si at or near the surface of the particles that resulted in localized intraband states. ${ }^{56}$ The presence of amorphous Si in similarly produced Si NPs was confirmed with ${ }^{29} \mathrm{Si} \mathrm{NMR} \mathrm{in} \mathrm{another} \mathrm{study.}{ }^{17}$ It was also shown that the Si NP size determined by TEM was considerably larger than the size determined by XRD line broadening, which has also been observed in our lab, similarly demonstrating that the Si NPs are crystalline with large amounts of structural defects. ${ }^{17,56}$

For the purposes of use in applications discussed in section 1.1, the Si NPs would ideally be stabilized in a colloidal solution before use. Thus, the Si NPs must be liberated from the $\mathrm{SiO}_{2}$ matrix via an aqueous $\mathrm{HF}$ etch. This is done by taking $300 \mathrm{mg}$ of composite and stirring it in a solution of $5 \mathrm{~mL} \mathrm{H} \mathrm{H}_{2} \mathrm{O}, 5 \mathrm{~mL}$ ethanol, and 5 
$\mathrm{mL} 48 \mathrm{wt} \% \mathrm{HF}$ in water for 1 hour according to equation 4 to remove the $\mathrm{SiO}_{2}$ and leave the surface of the Si NPs with hydrogen termination (H-Si NPs).

$$
\mathrm{SiO}_{2}+6 \mathrm{HF} \rightarrow \mathrm{H}_{2} \mathrm{SiF}_{6}+2 \mathrm{H}_{2} \mathrm{O}
$$

The etching process is terminated by phase transferring the non-polar H-Si NPs from the aqueous etching solution to a non-polar organic solvent (toluene, hexane, pentane, etc.). The particles can then be separated from the solvent via centrifugation, solvent discarded, and then re-suspended in a dry non-polar solvent of choice. The particles after this process tend to aggregate in solution and also show considerably less photoluminescence than the un-etched composite material (Figure $1.1 \mathrm{c}$ ).

\subsection{Motivation for the research presented herein}

While HF etching is commonly used to almost completely passivate dangling bonds (radicals) on Si surfaces, ${ }^{57,58}$ there is still a possibility of surface radicals being present after the HF etch. This has seen by others ${ }^{59}$ and has been demonstrated previously in our lab. ${ }^{60}$ Due to the reactive $\mathrm{H}-\mathrm{Si}$ surface bonds further passivation is usually conducted to stabilize the particles in solution, however this results in surface coverage that is incomplete, leaving reactive $\mathrm{Si}-\mathrm{H}$ bonds that could then go on to react with species that could alter the optical properties of the material. 
Because surface functionality can have such a large impact on the optical properties of the material, understanding of the processes by which optically active defects are produced on Si NP surfaces is imperative to better optimization of the desired optical property. It is, therefore, important to first observe changes to an ideal system like H-Si NPs before generalizing to other more complex systems.

The optical properties of semiconducting nanoparticles are first and foremost determined by two physical properties of the nanoparticle: particle size and crystallinity. While the literature has provided numerous methods for the development of particles with emergent properties, the ability to access highly crystalline particles from a scalable production method is still elusive.

This dissertation will first relate changes to the photoluminescence of $\mathrm{H}-\mathrm{Si}$ NPs and changes in surface composition to the exposure of these particles to different chemical environment. The results will then be used to demonstrate a novel surface passivation method that can be used to enhance the photoluminescence of Si NPs after HF etching. Finally, a new highly scalable method to produce Si NPs that are highly crystalline will be explored. 
Chapter 2: Effects of (2,2,6,6-Tetramethylpiperidin-1-yl)oxyl (TEMPO) and lighting conditions on the surface composition of hydride terminated silicon nanoparticles

\subsection{Introduction}

Silicon nanoparticles produced from the high temperature solid state reductive annealing method have relatively poor photoluminescence intensity upon liberation from the oxide matrix and subsequent hydride passivation. ${ }^{14}$ It has also been shown that alkylation of $\mathrm{H}-\mathrm{Si}$ NP surfaces by hydrosilylation with terminal alkenes and alkynes increases both PL brightness and colloidal stability in apolar solvents. ${ }^{15,39,40,53,61}$ We have hypothesized that one possible source of the poor luminescence intensity of $\mathrm{H}$-Si NPs is the presence of un-terminated Si surface sites with single unpaired electrons. ${ }^{15,60}$ For other types of semiconductor NPs, it is known that unsaturated surface sites (dangling bonds) result in non-radiative recombination of the photogenerated electron or hole that reduce the intrinsic, radiative electron/hole recombination at the band gap energy. The intrinsic radiative recombination of $\mathrm{H}$-Si NPs (termed here as the "core" emission) is expected when the Si crystalline grain size is below the Bohr exciton radius (approx. $5 \mathrm{~nm}$ ). For the approx. $5 \mathrm{~nm} \mathrm{H}$-Si NPs produced via the synthesis described above, we have previously attributed the broad photoluminescent emission maximum in the range of 600 to $700 \mathrm{~nm}$ as due to core emission based on the consistency with the effective mass approximation. ${ }^{15}$ 
To test this hypothesis the stable radical TEMPO was added to a solution of $\mathrm{H}$-Si NPs with the expectation that the TEMPO radical will form a bond to any radicals present on the Si NP surface and then prevent further oxidation by sterically blocking the surface and further stabilizing the particles in solution. An observed increase in PL intensity for the core emission would indicate that these surface radicals are responsible for the observed weak emission from the quantum confined core.

It was further hypothesized that the lighting conditions for the passivation could help to prevent over-oxidation of the surface. While the TEMPO radical is stable in solution, the $\mathrm{N}-\mathrm{O}$ bond is relatively weak and could be broken from the absorption of light with a wavelength below $600 \mathrm{~nm}$. If the $\mathrm{N}-\mathrm{O}$ bond is cleaved after passivating the surface radical it leaves the surface sterically accessible to further oxidation. To test the effects of lighting on surface passivation from TEMPO, samples were held in the dark, held under ambient lighting, and held under constant $365 \mathrm{~nm}$ UV irradiation for the duration of the passivation experiments.

\subsection{Experimental}

\subsubsection{Materials}

Trichlorosilane (Alfa Aesar), absolute ethanol (TCl), 49wt\% hydrofluoric acid in water (Sigma-Aldrich), toluene (acs reagent, Fisher), and (2,2,6,6tetramethyl-piperidin-1-yl)oxyl (TEMPO, C9H18NO, 98\%, Acros Organics) were 
purchased and used as received unless otherwise specified. Solvents that are referred to as "dry" were stored over $4 \AA$ molecular sieves for at least $24 \mathrm{hr}$. Solvents that are referred to as "degassed" were first purged with Ar for 20 minutes, followed by 3 freeze/pump/thaw cycles, and stored under argon atmosphere until use.

\subsubsection{Synthesis of H-Si NPS}

Hydride terminated Si NPs were synthesized using an established synthetic procedure. ${ }^{14,62}$ In a typical synthesis, a hydrolysis and polycondensation reaction of $5 \mathrm{~mL} \mathrm{HSiCl} 3$ upon the addition of $6 \mathrm{~mL}$ Millipore water forms a $\left[\mathrm{HSiO}_{1.5] \mathrm{n}}\right.$ polymer. Hydrolyzed polymer is then packed into an alumina crucible, transferred to a tube furnace (Lindberg Blue) equipped with a quartz tube, centered in the tube furnace, and placed under ultra-high purity $\mathrm{N}_{2}$ at a flow rate of approximately 50 $\mathrm{mL} / \mathrm{min}$. The $\left[\mathrm{HSiO}_{1.5}\right]_{\mathrm{n}}$ polymer was then heated at a rate of $20^{\circ} \mathrm{C} / \mathrm{min}$ to $150^{\circ} \mathrm{C}$ where it was held for 2 hours to ensure dryness. The temperature was then increased at $20^{\circ} \mathrm{C} / \mathrm{min}$ to a maximum temperature of $1100^{\circ} \mathrm{C}$ and was then held for ten hours, followed by cooling to room temperature at $10^{\circ} \mathrm{C} / \mathrm{min}$. This results in a solid, composite material composed of crystalline $\mathrm{Si}^{0}$ domains within an $\mathrm{SiO}_{2}$ matrix. The solid, composite was then ball milled in a high energy mill mixer (SPEX $8000)$ for 1 minute to homogenize the composite material and increase its surface area; milled composite material was then stored under $\mathrm{Ar}$ for future use. The $\mathrm{Si}^{0}$ crystallites were liberated from the $\mathrm{SiO}_{2}$ matrix by first dispersing $300 \mathrm{mg}$ of the 
milled composite into a solution containing $5 \mathrm{~mL}$ of absolute ethanol and $5 \mathrm{~mL}$ of Millipore water. This mixture was sonicated for 1 minute to disperse the solid, and then $5 \mathrm{~mL}$ of $48 \mathrm{wt} \% \mathrm{HF}$ aqueous solution was added. The resulting suspension was stirred in the dark for 1 hour. Following the HF etching to remove the oxide matrix, the $\mathrm{Si}^{0}$ particles are hydrophobic due to hydride surface passivation; hereafter, we call these hydride-terminated Si NPs (H-Si NPs). The hydrophobic $\mathrm{H}$-Si NPs were then phase transferred from the aqueous HF suspension into toluene by thoroughly mixing the two liquids and subsequently allowing for phase separation, followed by removal of the organic layer via pipet. The extracted organic layer was then centrifuged at $2500 \mathrm{rpm}$ in glass centrifuge tubes, the supernatant removed, and the pellet containing the hydrophobic particles redispersed in dry toluene. The centrifugation step was repeated two more times to remove ethanol, water, and any residual HF. The purified H-Si NPs were then redispersed into dry and degassed toluene at an approximate concentration of 0.1 $\mathrm{mg} / \mathrm{mL}$ and transferred to a $\mathrm{N}_{2}$ filled glovebox until further use.

\subsubsection{TEMPO surface treatment}

For this set of experiments, the photophysical properties of the H-Si NPs in colloidal solution were measured under a total of nine different environmental conditions. To prepare the $\mathrm{H}-\mathrm{Si}$ NP samples for analysis, $(2,2,6,6-$ Tetramethylpiperidin-1-yl)oxyl (TEMPO) solutions at concentrations of $2 \mathrm{mM}$ and $10 \mathrm{mM}$ in toluene were first prepared in a nitrogen filled glove box. Then, $5 \mathrm{~mL}$ of 
the TEMPO solutions were added to three individual $20 \mathrm{~mL}$ air free scintillation vials (three each at two concentrations). A set of control samples was also prepared by adding $5 \mathrm{~mL}$ of toluene to three individual $20 \mathrm{~mL}$ air free scintillation vials. To each of the nine vials, a $5 \mathrm{~mL}$ aliquot of the H-Si NPs in toluene was added to result in suspensions with equal concentrations of H-Si NPs (approx. 0.05 $\mathrm{mg} / \mathrm{mL}$ ) and varying concentrations of TEMPO. One set of H-Si NP samples with variable TEMPO concentration (control, 1 and $5 \mathrm{mM}$ ) was then held in the dark, a second set under ambient lighting (overhead fluorescent and natural light), and a third set under constant $365 \mathrm{~nm}$ irradiation (14.4 W). Aliquots were taken from each sample at time points ranging from 0 up to 44 hours to measure the steady state photoluminescence spectra.

\subsubsection{Photoluminescence measurements and analysis}

Steady state photoluminescence (PL) measurements were conducted on a Shimadzu 5301-RFPM spectrophotometer equipped with a Xe laser source. All measurements were conducted using $365 \mathrm{~nm}$ excitation. At each timepoint, approx. $1 \mathrm{~mL}$ aliquots were taken and added to fused quartz cuvettes via syringe injection through the septum cap. Due to the tendency of the H-Si NPs to aggregate, considerable turbidity of the samples was observed, which registered in the PL spectra as high intensity scattered light at short wavelength that exponentially decreases with increasing wavelength. Also, due to the diffraction grating used for excitation wavelength selection from the source, the second order 
diffraction for the $365 \mathrm{~nm}$ excitation is observed at $730 \mathrm{~nm}$, again due to scattering from the sample. In order to better monitor the long wavelength photoluminescence of the H-Si NPs a filter set was used on the source and detector side of the sample. A $365 \mathrm{~nm}$ band pass filter was used on the source side to prevent the passage of the $730 \mathrm{~nm}$ light from the diffraction grating. A 450 $\mathrm{nm}$ long pass filter was used on the detector side to prevent shorter wavelength light from saturating the detector. The raw PL spectral curves were then simulated using peak fitting software (MagicPlot 2.7.2) to model the spectra. The fitting procedure is thoroughly discussed for representative spectra in the Results section.

\subsubsection{FTIR spectroscopy}

FTIR measurements were conducted on a ThermoFisher Nicolet iS10 FTIR spectrometer with using a diamond-window single bounce ATR attachment. A background spectrum was taken prior to each sample spectrum and subtracted using the instrument software package. FTIR spectra were measured initially and after the conclusion of PL spectroscopy experiments ( $>44$ hours), with no data acquisition at intermediate timepoints due to sample preparation time limitations. The initial FTIR spectrum was taken of the stock H-Si NP colloid after purification; the sample for this measurement was prepared by repeatedly drop casting the $\mathrm{H}$ Si NP stock onto the ATR crystal surface and allowing the solvent to evaporate. This process was sped up by using an air gun blowing a slow stream of room 
temperature air. For FTIR measurements following PL spectroscopy, the H-Si NP samples containing TEMPO were first purified to separate any (unbound) TEMPO from the Si NPs. To accomplish this, the Si NPs were centrifuged out of their respective TEMPO solutions, the supernatant was discarded, and the Si NPs were re-dispersed in dry toluene. This procedure was repeated three times to remove as much (excess) TEMPO as possible in order to simplify FTIR spectral analysis. After purification, these Si NP samples were then drop cast onto the ATR crystal and FTIR measurements were taken as described for the stock H-Si NP sample above. All presented spectra were normalized to the most intense peak in the set of spectra.

\subsubsection{X-ray photoelectron spectroscopy (XPS) measurements}

Samples were prepared by drop casting the same Si NP samples used for FTIR measurements onto aluminum foil. The XPS sample of the H-Si NP stock (initial condition) was prepared over 44 hours before the experimental Si NP XPS samples and was meanwhile held under vacuum until measurement. XPS measurements were conducted on a Versaprobe II (Physical Electronics Inc., PHI, Chanhassen, MN) operating at $15 \mathrm{kV}$ and $25 \mathrm{~W}$, with a monochromatic Al Ka Xray beam (100 $\mu \mathrm{m}$ spot size). Detailed scans of the $\mathrm{Si}(2 \mathrm{p})$ peak were conducted with a pass energy of $20 \mathrm{eV}$, speed of $40 \mathrm{~ms} / \mathrm{step}$, and with a step size of $0.1 \mathrm{eV}$. 3 scans total were conducted and averaged for each sample. The XPS spectra were analyzed using Multipak software suite. First the spectra were calibrated by 


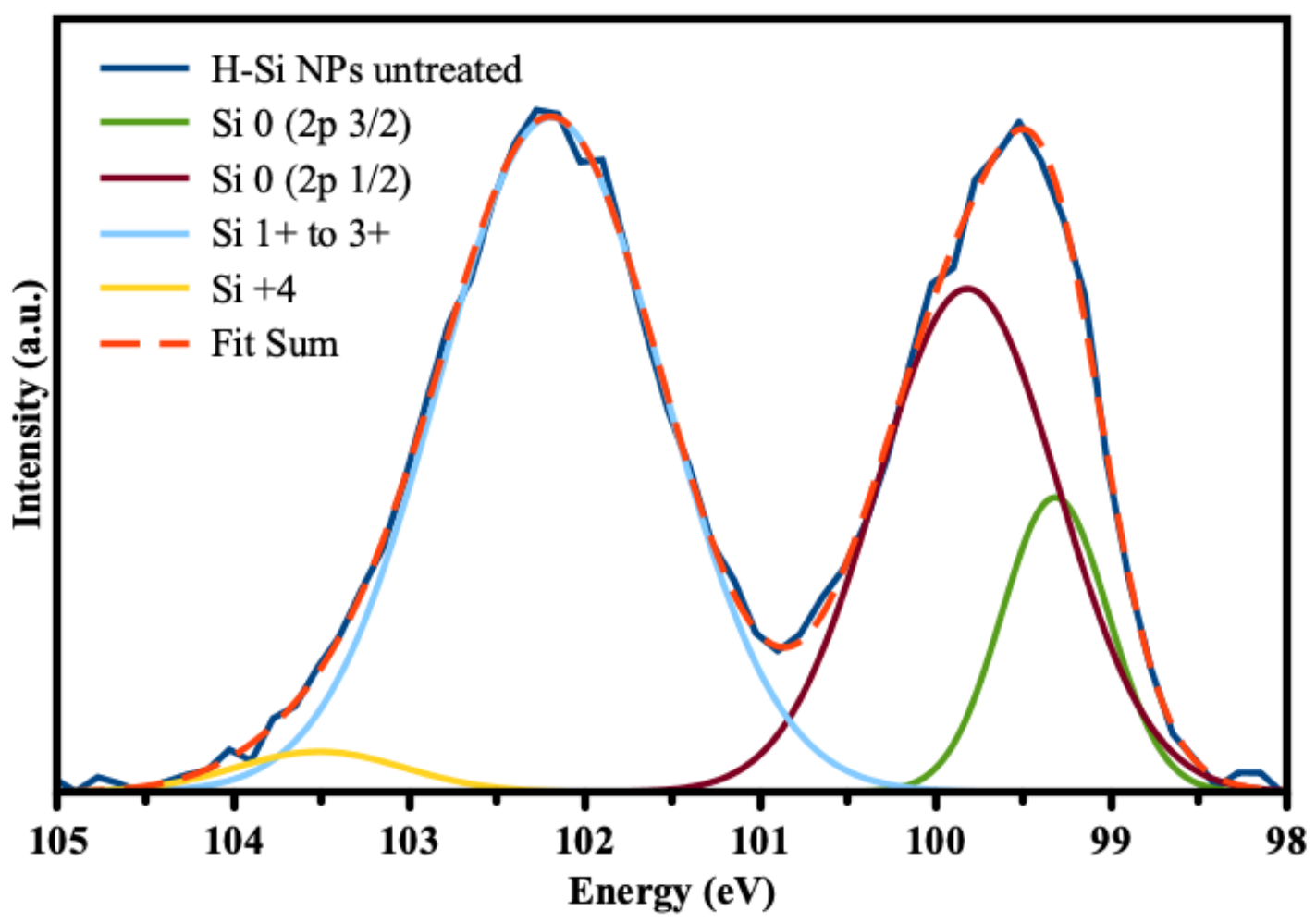

Figure 2.1: Binding energy in the $\mathrm{Si}(2 \mathrm{p})$ region for $\mathrm{H}-\mathrm{Si}$ NPs before treatment (dark blue trace). The Gaussian curves added represent the Si oxidation states of 0 (green and dark red), 1+ to 3+ (light blue), and 4+ (yellow).

shifting to align the $\mathrm{C}(1 \mathrm{~s})$ peak at $284.9 \mathrm{eV}$. The $\mathrm{Si}(2 \mathrm{p})$ peak was then modeled using a literature procedure where four Gaussian curves were used. ${ }^{63,64}$ An example of the modeling can be seen in Figure 2.1.

The XPS spectrum of the H-Si NPs prior to treatment (Figure 2.1) has a large doublet centered at a binding energy of $99.5 \mathrm{eV}$; this doublet is assigned as the overlapping spin-orbit components $\mathrm{Si}\left(2 \mathrm{p}_{3 / 2}\right)$ and $\left(2 \mathrm{p}_{1 / 2}\right)$ of silicon in the elemental state $\left(\right.$ i.e., $\left.\mathrm{Si}^{0}\right) .{ }^{63,64} \mathrm{In}$ this spectrum, there is also a broad peak at higher binding energy that can be broken down into two Gaussian peaks at approx. 102 
$\mathrm{eV}$ representing partially oxidized $\mathrm{Si}$ (oxidation state between $1+$ and $3+$ ) and at approx. $103.5 \mathrm{eV}$ that represents fully oxidized $\mathrm{Si}^{4+}$ (as in $\mathrm{SiO}_{2}$ ).

\subsubsection{Raman spectroscopy}

Raman spectroscopy was conducted on a Horiba HR800 UV equipped with a $100 \mathrm{~mW} 532 \mathrm{~nm}$ diode-pumped solid-state laser. The samples used for Raman spectroscopy were identical to those used for XPS measurements, namely the Si NPs of interest drop cast onto aluminum foil. The Si NP samples were held under vacuum between XPS and Raman measurements to prevent any oxidation of the Si NPs from the atmosphere. Each sample was measured in five spots, following a procedure used previously for the Raman analysis of H-Si NPs produced by our lab. ${ }^{56}$ Each spot first had the linear sloping background subtracted, then was normalized to the crystalline Si peak at approx. $520 \mathrm{~cm}^{-1}$, then all normalized spot measurements for the sample were averaged. MagicPlot software (version 2.7.2) was used to model the averaged spectra in the 400 to $600 \mathrm{~cm}^{-1}$ range, where peaks due to crystalline and amorphous silicon would be expected. Three Gaussian curves were used to represent the LO and TO modes from crystalline silicon (c-Si) and the TO mode from amorphous silicon $(a-S i) .65$ Percent crystallinity was calculated using the integrated intensity of the $c$-Si TO peak divided by the sum of the integrated intensities of the c-Si and a-Si TO peaks. 


\subsection{Results}

\subsubsection{H-Si NP PL spectra as a function of TEMPO concentration and lighting environment}

With three lighting conditions and three TEMPO concentrations, a total of nine samples were prepared to observe nine total TEMPO/lighting conditions; three of these were control samples with no TEMPO. Steady state PL spectroscopy was used to correlate the effects of the H-Si NP solution environment with the temporal evolution of the H-Si NP PL spectra.

A PL spectrum for each of the nine samples was measured at time points of $0.5,1,2,4,21,28$, and 44 hours, producing a time series of spectra to be analyzed. An initial spectrum for each sample was also taken at $\mathrm{t}=0 \mathrm{hr}$; in each case, the sample was prepared from the stock H-Si NP solution, which was diluted to the same concentration as the rest of the samples in the respective sets. These spectra were acquired prior to TEMPO addition to any of the samples and are provided in the following data sets to permit analysis of change over time as a function of sample conditions. The samples were kept under constant stirring between data collection time points. Under constant stirring the H-Si NP solution concentration can be assumed to be the same for all aliquots taken, and thus comparisons of absolute PL intensity can be assumed to be valid across conditions. However, H-Si NPs have a strong tendency to aggregate in most solvents, which would be expected to result in increased scattering intensity and decreased photoluminescence emission intensity. This tendency, for the purposes 

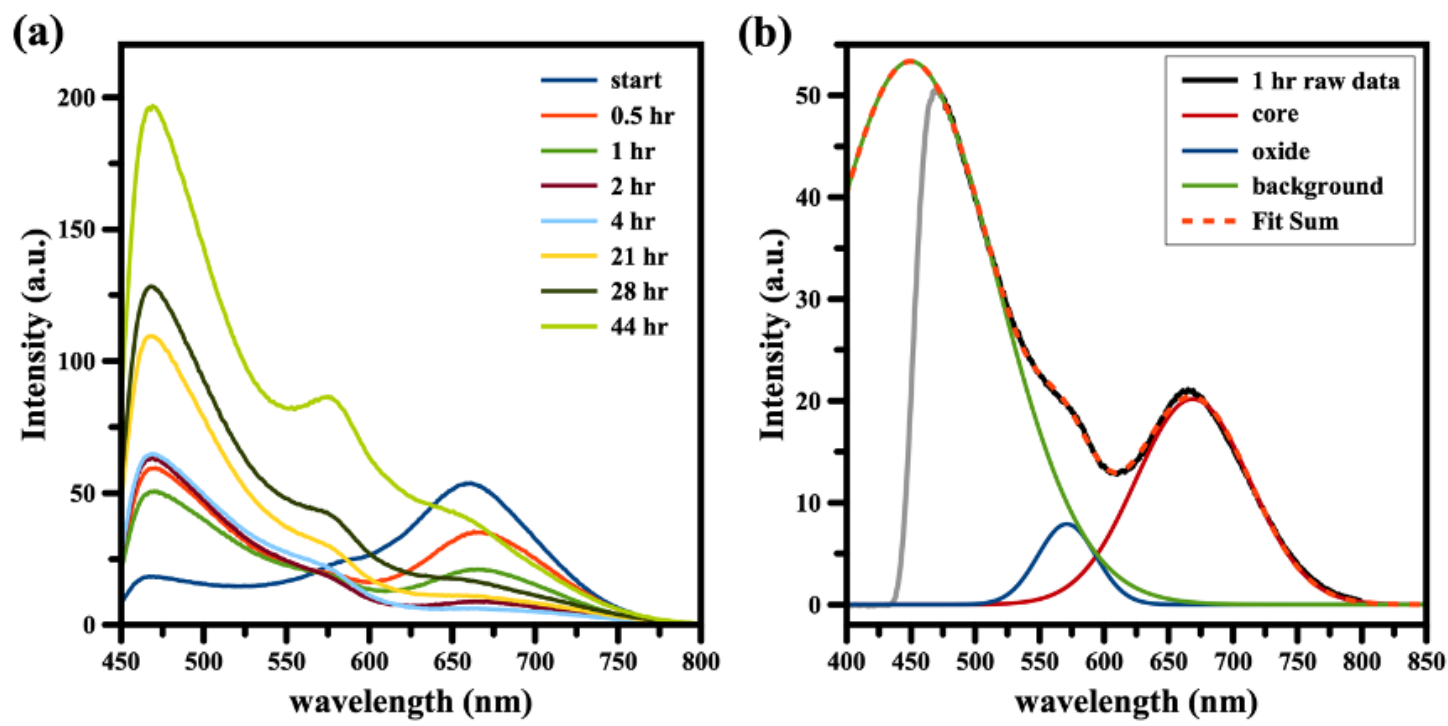

Figure 2.2: PL spectra for the control sample and fit plot analysis of one time point. (a) PL spectra collected over 44 hours for the control sample (no TEMPO) held in the dark, showing the changes to the PL spectra over the time points indicated. (b) The Fit plot analysis of the PL spectrum for the 1 hour time point, modeled by three Gaussian curves representing the "core" emission (red line), the "oxide" emission (blue line), and the "background" scattering or blue emission (green line).

of this study, will be assumed to be minimal due to constant agitation of the samples and is modeled in our analysis. We also used filters to minimize the contributions of scattering to the photoluminescence spectra measured and presented herein.

The PL spectra collected over time from the control H-Si NP solution (with no TEMPO) held in the dark condition (control/dark) are shown in Figure 2.2a and are presented to demonstrate the analysis of one of the nine conditions independently. At $t=0 \mathrm{hr}$, the spectrum of the $\mathrm{H}-\mathrm{Si}$ NP solution excited at $365 \mathrm{~nm}$ shows the long pass filter cut on at $450 \mathrm{~nm}$ followed by a gradual decrease in 
intensity, then a shoulder at $577 \mathrm{~nm}$ and then a broad peak at $662 \mathrm{~nm}$. After the first 30 minutes there is a decrease in intensity for the $662 \mathrm{~nm}$ peak, no change in the $577 \mathrm{~nm}$ peak intensity, and an increase in the background intensity.

For the spectra taken after 1, 2 and 4 hours, the peak wavelength appears to be maintained at $662 \mathrm{~nm}$ peak, but the emission intensity decreases to an almost fully quenched state by 4 hours. In general, larger spectral changes were observed at longer time points, after the samples were allowed to stir overnight. For example, at 21 hours the control/dark sample shows small increases in intensity at both $662 \mathrm{~nm}$ and $577 \mathrm{~nm}$, and the background intensity has also further increased. Further monitoring at 28 hours and 44 hours showed further increases in intensity of all spectral bands and a possible blue shift in the core emissive wavelength from $662 \mathrm{~nm}$ to approx. $650 \mathrm{~nm}$ after 44 hours.

The integrated intensity and wavelength of maximum emission intensity $\left(\lambda_{\max }\right)$ for each emissive component can be calculated using curve fitting software as described in the methods section. The intensity is measured in counts per second (cps), thus the integrated intensity technically has units of $c p s x n m$. The physical meaning of these units can be described as related to the total counts measured over a spread of wavelengths that are attributed to the emissive state of interest. For the purposes of these experiments, we will denote these units as arbitrary units (a.u.) since they are meant to only give a relative value to each sample measurement that can then be used for comparison to other samples. The raw data represented by the black line in Figure 2.2b is from the 1 hour time point 
measurement shown in Figure 2.2a for the control/dark sample. The raw data is simulated using three Gaussian curves and the sum of these curves is shown as the orange dashed line, in good agreement with the raw data. Based on prior literature and our set of observations across the samples, we tentatively assigned the $662 \mathrm{~nm}$ peak as due to the quantum confined core (component labeled "core"), the $577 \mathrm{~nm}$ peak as originating from an oxide related surface state (component labeled "oxide"), and the short wavelength features (labeled "background") as due to aggregation-induced scattering. The latter component is likely mostly due to scattering but could also include any possible shorter wavelength emission from the sample, of any origin.

For the purposes of this experiment, we were mostly concerned with the emission from the quantum confined core and the effect that lighting and TEMPO concentrations have on its intensity. Thus, the temporal PL spectral analyses in this chapter focus on the position and intensity of the core emissive component, and we will ignore contributions from the oxide and background to the overall spectra in initial analyses. The following figures show the changes to the $\lambda$ max 

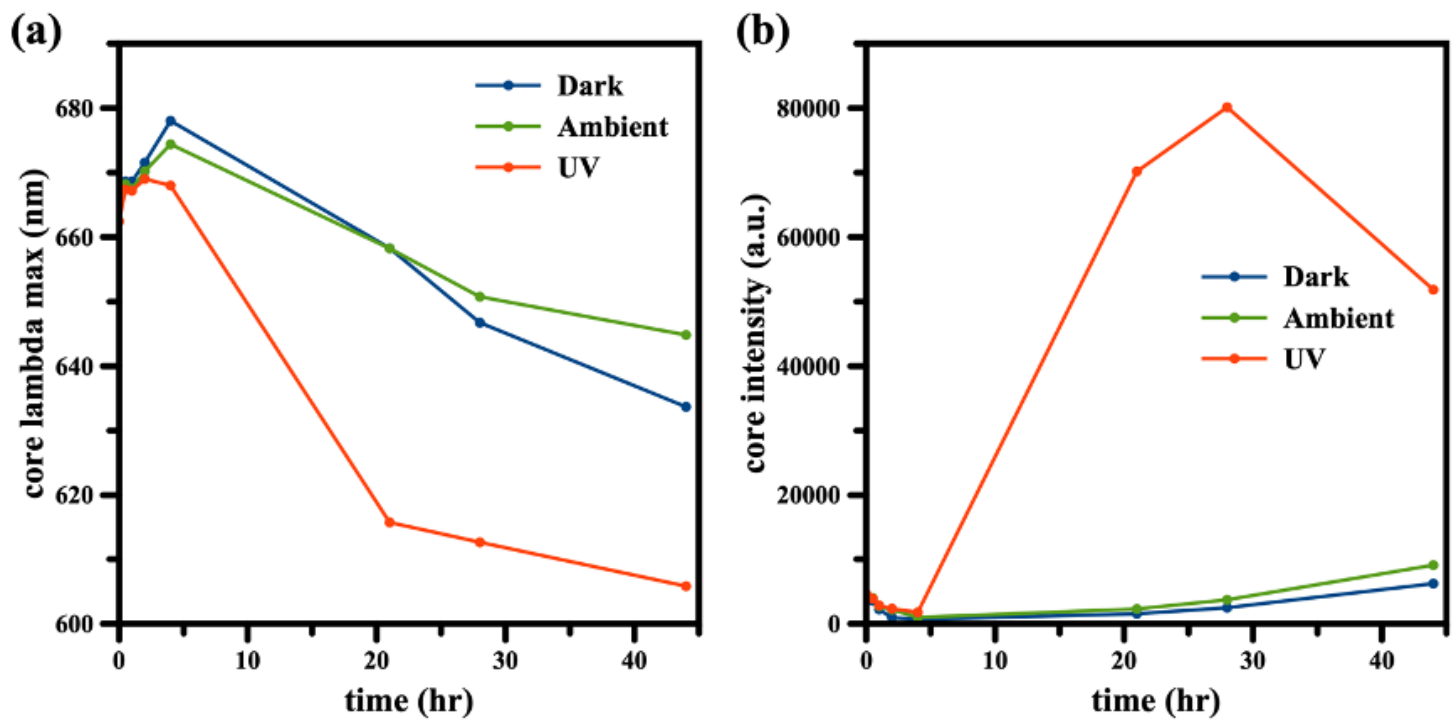

Figure 2.3: PL intensity and lambda max for the control samples. (a) Core lambda max and (b) integrated PL intensity as a function of time for each of the indicated lighting conditions for the control samples with no TEMPO.

position and the integrated intensity for the "core" emission from the curve fitting analysis for each TEMPO concentration.

The core PL $\lambda_{\max }$ for the control H-Si NP samples containing no TEMPO (Figure 2.3a) initially increased over the first 4 hours from $662 \mathrm{~nm}$ to $668 \mathrm{~nm}$ for the sample under UV light, $674 \mathrm{~nm}$ for the sample under ambient light, and 678 $\mathrm{nm}$ for the sample held in the dark. This initial period was then followed by a steady decrease in $\lambda_{\max }$ for all samples, to final wavelengths of $605 \mathrm{~nm}, 645 \mathrm{~nm}$, and 634 $\mathrm{nm}$ for the UV, ambient, and dark lighting conditions, respectively, at 44 hours.

The integrated PL intensity for the samples containing no TEMPO (Figure 2.3b) all decreased in intensity from 4600 a.u. at $t=0 \mathrm{hr}$ to 1800,1000 , and 800 a.u. at 4 hours for the UV, ambient, and dark lighting conditions, respectively. At 
much longer time points, a dramatic increase in intensity is observed only for the sample held under UV light. For the control/UV sample, a maximum intensity of 80100 a.u. is reached after 28 hours (17 times the initial intensity), followed by a decrease to a final intensity of 51800 a.u. at 44 hours, 11 times higher than the initial value. Conversely, the core PL integrated intensity of the sample held in ambient light gradually increased after 4 hours, from 1000 a.u. at 4 hours to 9100 at 44 hours. Similar to the control/ambient sample, the sample held in the dark showed a gradual increase in core PL integrated intensity, from 800 a.u. at 4 hours to 6300 a.u. at 44 hours.

Overall, there was little spectral difference between H-Si NP samples held under ambient and dark lighting conditions in the absence of TEMPO, with respect to the changes in emission wavelength and intensity over time. There was, however, a large change in emission intensity that occurred between 4 and 21 hours in the UV irradiated control sample and that increase in intensity was coupled to a large decrease in emission wavelength. This indicates not only that continuous UV irradiation can produce pronounced changes to the emission of $\mathrm{H}$ Si NPs, but also that there is a saturation point, after which UV-induced bleaching occurs. 

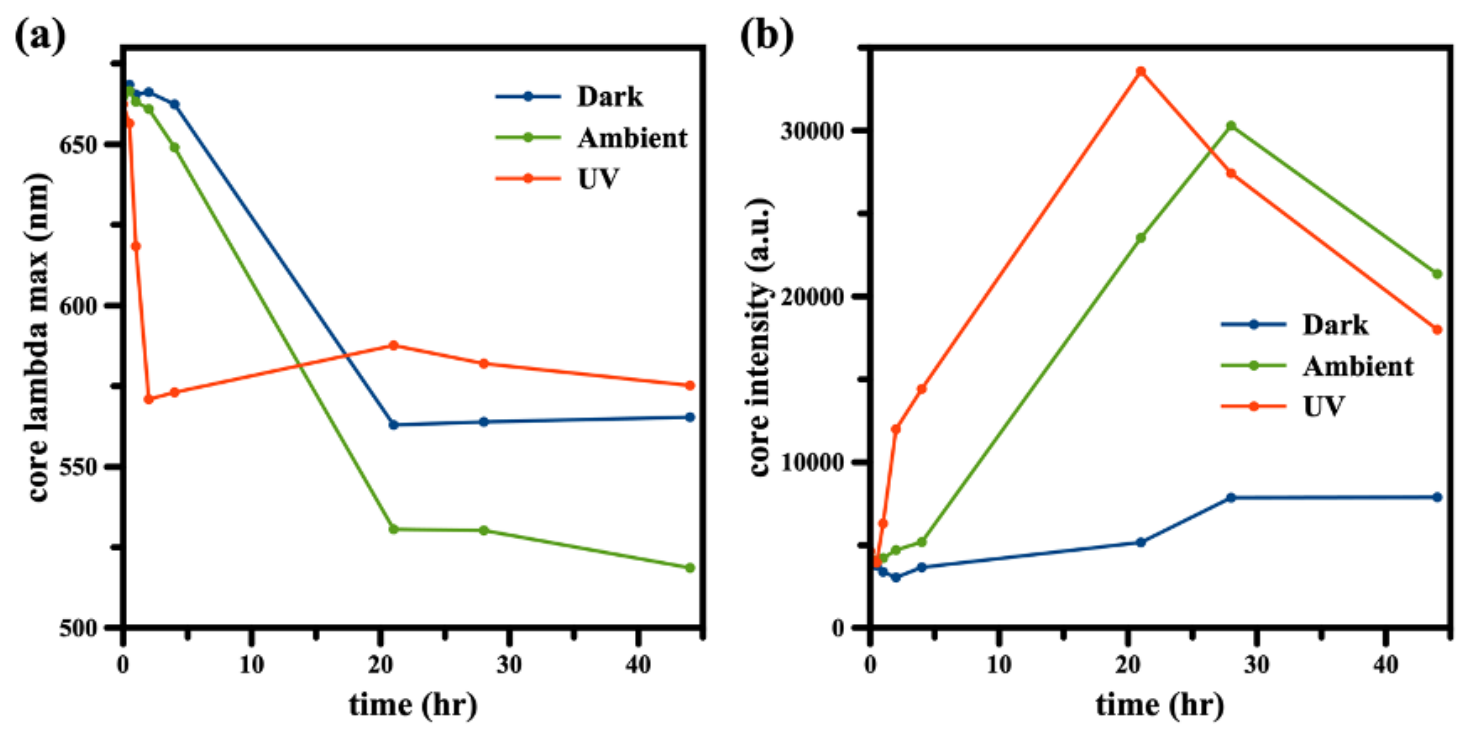

Figure 2.4: PL intensity and lambda max for the $1 \mathrm{mM}$ TEMPO samples. (a) core lambda max and (b) integrated PL intensity as a function of time for each lighting condition of the $1 \mathrm{mM}$ TEMPO treated samples.

The H-Si NPs in $1 \mathrm{mM}$ TEMPO also showed overall decreases in core $\lambda$ max (Figure 2.4a) and increases in core PL intensity (Figure 2.4b) for all lighting conditions. The core $\lambda_{\max }$ for the sample treated with $1 \mathrm{mM}$ TEMPO and held under UV light decreased from $662 \mathrm{~nm}$ to $571 \mathrm{~nm}$ over the first 2 hours and afterwards remained relatively constant; at 44 hours, the $\lambda \max$ was $575 \mathrm{~nm}$. A more gradual initial decrease in $\lambda_{\max }$ was observed for the $1 \mathrm{mM} /$ ambient, and $1 \mathrm{mM} /$ dark samples. For the $1 \mathrm{mM} /$ ambient sample, the $\lambda \max$ initially decreased from $662 \mathrm{~nm}$ to $649 \mathrm{~nm}$ over the first 4 hours, followed by a large decrease to $530 \mathrm{~nm}$ at 21 hours; at 44 hours, the $\lambda_{\max }$ was $519 \mathrm{~nm}$. For the $1 \mathrm{mM} /$ dark sample, the $\lambda_{\max }(662$ nm) remained relatively constant over the first 4 hours, followed by a large decrease to $563 \mathrm{~nm}$ at 21 hours. At 44 hours, the $\lambda \max$ was $565 \mathrm{~nm}$ for the 
$1 \mathrm{mM} /$ dark sample, as compared to $519 \mathrm{~nm}$ for the $1 \mathrm{mM} /$ ambient sample and 575 $\mathrm{nm}$ for the $1 \mathrm{mM} / \mathrm{UV}$ sample.

The integrated PL intensity (Figure $2.4 \mathrm{~b}$ ) of the $1 \mathrm{mM} / \mathrm{UV}$ sample decreased initially from 4600 a.u to 3900 a.u within the first 30 minutes, followed by an increase to a maximum at 33600 a.u after 21 hours, and finally, a decrease to 18000 a.u at 44 hours. Similarly, the integrated PL intensity of the $1 \mathrm{mM} / \mathrm{ambient}$ sample decreased from 4600 a.u to 4000 a.u initially, followed by an increase to a maximum of 30300 a.u at 28 hours, and finally, a decrease to 21300 a.u at 44 hours. Conversely, the temporal change in the integrated PL intensity of the $1 \mathrm{mM} /$ dark sample was different, and overall less pronounced in comparison to the lightexposed samples. The integrated PL intensity of the $1 \mathrm{mM} /$ dark sample decreased in intensity from 4600 a.u to 3000 a.u over the first 2 hours, followed by a gradual increase over the remainder of the experiment to a final intensity of 7900 a.u at 44 hours.

Overall, for the H-Si NP samples in $1 \mathrm{mM}$ TEMPO solution, a decrease in the core emission $\lambda_{\max }$ and an increase in the core emission intensity was observed, regardless of the lighting conditions. However, the rate at which the initial $\lambda_{\max }$ decrease occurred was observed to be correlated with the lighting conditions; the rate of change of the core PL $\lambda_{\max }$ was slowest for the sample held in the dark and fastest for the sample held under UV light. Additionally, the core PL integrated intensity increase was observed to be most rapid and pronounced for the sample held under UV light. Both the rate and magnitude of change were 

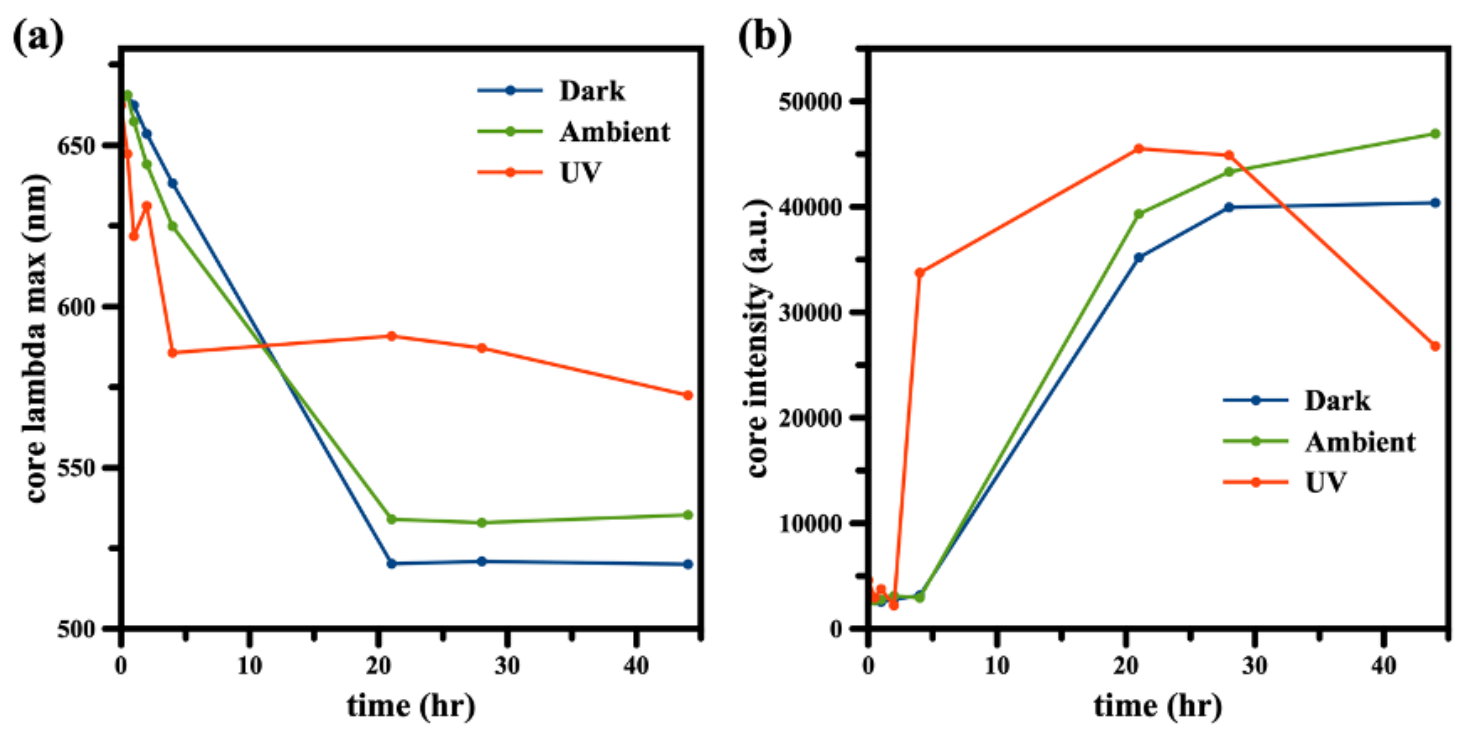

Figure 2.5: PL intensity and lambda max for the $5 \mathrm{mM}$ TEMPO samples. (a) core lambda max and (b) integrated PL intensity as a function of time for each lighting condition of the $5 \mathrm{mM}$ TEMPO treated samples.

observed to be smaller for the sample held under ambient conditions, and yet smaller for the sample held in the dark.

The H-Si NPs in $5 \mathrm{mM}$ TEMPO also showed overall decreases in core $\lambda$ max (Figure 2.5a) and overall increases in core PL intensity (Figure 2.5b) with time for all lighting conditions. In all three lighting conditions in 5 mM TEMPO, dramatic, initial decreases in the core PL $\lambda_{\max }$ of the H-Si NP samples were observed. In the first four hours of the experiment, the H-Si NP sample held under UV light exhibited the most rapid initial decrease in $\lambda_{\max }$, and the sample held in the dark showed the least rapid initial change in $\lambda_{\max }$. Over the full time interval measured, the $\lambda_{\max }$ for the $5 \mathrm{mM} / \mathrm{UV}$ sample initially decreased from $662 \mathrm{~nm}$ to $585 \mathrm{~nm}$ in the first 4 hours, and afterwards remained fairly constant, decreasing slightly to $572 \mathrm{~nm}$ at 44 hours. 
In comparison, for both the $5 \mathrm{mM} /$ ambient and $5 \mathrm{mM} /$ dark samples with slower initial rates of blue shifting, plateaus of the PL $\lambda \max$ were reached at 21 hours and afterwards maintained for the remainder of the experiment at 534 and $520 \mathrm{~nm}$, respectively.

The integrated PL intensity of the core emission was also plotted as a function of time (Figure 2.5b). The intensity for all three samples remained relatively constant for the first 2 hours of the experiment. For the $5 \mathrm{mM} / \mathrm{UV}$ sample, a very large increase in the PL intensity was observed at the 4 hour timepoint, while the PL intensity of the $5 \mathrm{mM} /$ ambient and $5 \mathrm{mM} /$ dark samples remained constant. However, at 21 hours all three samples showed similarly large increases in the core PL integrated intensity, to 45500 a.u., 39300 a.u., and 35200 a.u. for the $5 \mathrm{mM} / \mathrm{UV}, 5 \mathrm{mM} / \mathrm{ambient}$, and $5 \mathrm{mM} /$ dark samples, respectively. After 21 hours, only the $5 \mathrm{mM} / \mathrm{UV}$ sample was observed to undergo a decrease in intensity, to a final value of 26800 a.u. at 44 hours, which is 6 times higher as compared to the sample at $\mathrm{t}=0 \mathrm{hr}$. Conversely, the $5 \mathrm{mM} /$ ambient and $5 \mathrm{mM} /$ dark samples continued to increase in core PL integrated intensity for the remainder of the observed time with the $5 \mathrm{mM} /$ ambient reaching a maximum of 46900 a.u. or 10 times greater than the starting emission intensity.

Although the PL for all samples treated with TEMPO did increase in intensity, a blue shift of the core emission lambda max to below $600 \mathrm{~nm}$ was also observed. Similarly, the constant $365 \mathrm{~nm}$ UV irradiation increased the PL intensity for all samples including the control with no TEMPO. In all cases, an overall 
increase in PL intensity was observed in conjunction with a blue shift of the emission wavelength. These observations are consistent with oxidation-induced shrinkage of the elemental crystalline silicon cores when they are in TEMPO solution or stored under light. Thus, we proceeded to characterize these samples using FTIR, XPS, and Raman spectroscopies to observe compositional changes, such as oxidation, that may occur with time and vary with the sample conditions.

\subsubsection{FTIR measurements before and after TEMPO treatment}

The infrared absorption spectrum was measured for each sample condition before and after the steady state PL spectroscopy study in order to assess whether the temporal PL changes were associated with changes in (surface) composition. In all of the following figures, the FTIR spectrum of the samples before treatment is presented as a reference, however it is the same spectrum in all cases. The FTIR spectra for the control samples without TEMPO held in different lighting conditions are presented in Figure 2.6 (following page). Before treatment there are two main vibrational modes observed at $2100 \mathrm{~cm}^{-1}$ and $910 \mathrm{~cm}^{-1}$ that are assigned to $\mathrm{Si}-\mathrm{H}$ stretching and scissoring modes respectively (Si-H wag at approx. $600 \mathrm{~cm}^{-1}$ not shown due to scan window), and a small broad feature centered at $1080 \mathrm{~cm}^{-1}$ assigned to Si-O-Si stretching.

The Si-H surface bonding features are retained for the control/dark sample up to 44 hours; however the Si-O-Si stretching at $1080 \mathrm{~cm}^{-1}$, Si-O-Si bending at $880 \mathrm{~cm}^{-1}$, and $\mathrm{Si}-\mathrm{OH}$ stretching at $800 \mathrm{~cm}^{-1}$ are all clearly stronger than before 


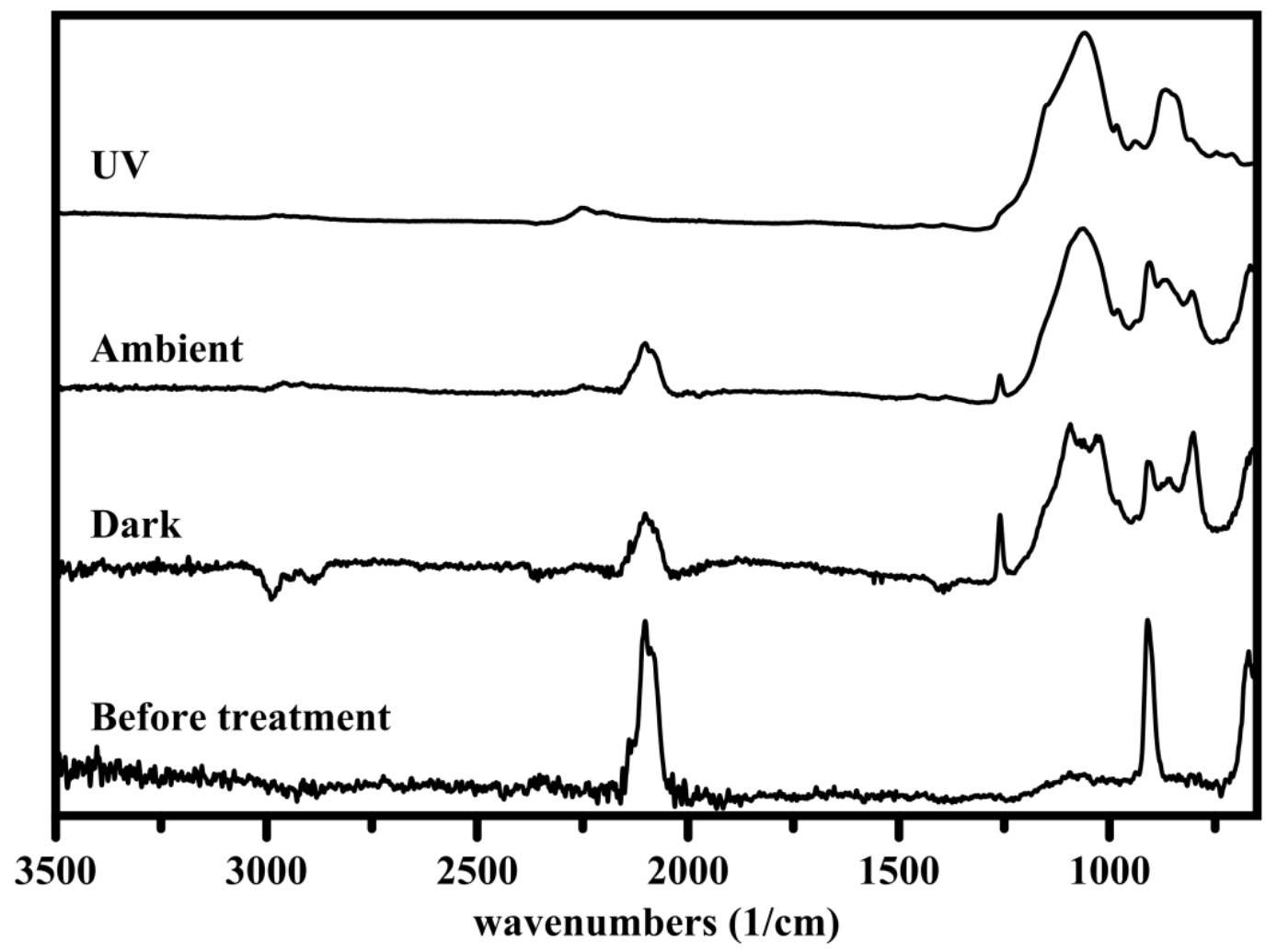

Figure 2.6: FTIR spectra for set of control samples (no TEMPO). Traces (from bottom to top) are H-Si NPs prior to treatment, held in the dark, ambient lighting, and $365 \mathrm{~nm}$ irradiation.

treatment. Further, for this sample, there is also a sharp peak at $1260 \mathrm{~cm}^{-1}$ that can be assigned to Si-C stretching. ${ }^{15,39,66}$ It should be noted for the control/dark FTIR spectrum that there is an over-subtraction of the background that can be seen in the 3000 to $2800 \mathrm{~cm}^{-1}$ region and is also observed centered at $1060 \mathrm{~cm}^{-1}$. Similarly, the control/ambient FTIR spectrum also retained Si-H stretching and scissoring modes throughout the 44 hour experiment, accompanied by an increase in the Si-O-Si stretching at $1080 \mathrm{~cm}^{-1}$. However, for this sample there is an additional shoulder at $1150 \mathrm{~cm}^{-1}$ that can be assigned to more complex $\mathrm{Si}-\mathrm{O}-\mathrm{Si}$ 
cage like structures ${ }^{62,67}$ and the Si-O-Si bending $\left(880 \mathrm{~cm}^{-1}\right)$ and Si-OH $\left(800 \mathrm{~cm}^{-1}\right)$ stretching modes are also clearly visible. Finally, for the control/ambient FTIR spectrum after 44 hours, a peak at $1260 \mathrm{~cm}^{-1}$ indicative of $\mathrm{Si}-\mathrm{C}$ bonding is also present. By comparison, the FTIR spectrum of the control/UV sample after 44 hours of constant exposure to $365 \mathrm{~nm}$ irradiation showed a complete elimination of the $\mathrm{Si}-\mathrm{H}$ related vibrations, with only $\mathrm{Si}-\mathrm{O}-\mathrm{Si}$ and $\mathrm{Si}-\mathrm{OH}$ being clearly present. The weak feature observed at approx. $2250 \mathrm{~cm}^{-1}$ is due to atmospheric $\mathrm{CO}_{2}$.

Overall, the control/dark and control/ambient samples showed similar relative intensities for the $\mathrm{Si}-\mathrm{H}$ and the $\mathrm{Si}-\mathrm{O}-\mathrm{Si}$ stretching modes, as well as $\mathrm{Si}-\mathrm{C}$ stretching at $1260 \mathrm{~cm}^{-1}$; the latter feature has been observed by previous members of our lab and identified as a possible interaction with the solvent. ${ }^{60}$ The retained presence of the $\mathrm{Si}-\mathrm{H}$ stretching modes indicates that the entire surface of the particles has not been oxidized under these conditions. However, the increase in Si-O-Si stretching indicates that some oxidation that has occurred over time, even in the presumed absence of $\mathrm{H}_{2} \mathrm{O}$ and $\mathrm{O}_{2}$. A comparatively greater extent of surface oxidation occurs for the control/UV sample, for which Si-H stretching is not observed; again, these oxidation features arise in the presumed absence of atmospheric/environmental oxygen sources.

The FTIR spectra of the H-Si NP samples following 44 hours in $1 \mathrm{mM}$ TEMPO solution and held in different lighting conditions are presented in Figure 2.7 (following page). Regardless of lighting condition, all of the $\mathrm{Si}-\mathrm{H}$ related vibrational modes that were initially present have disappeared, accompanied by 


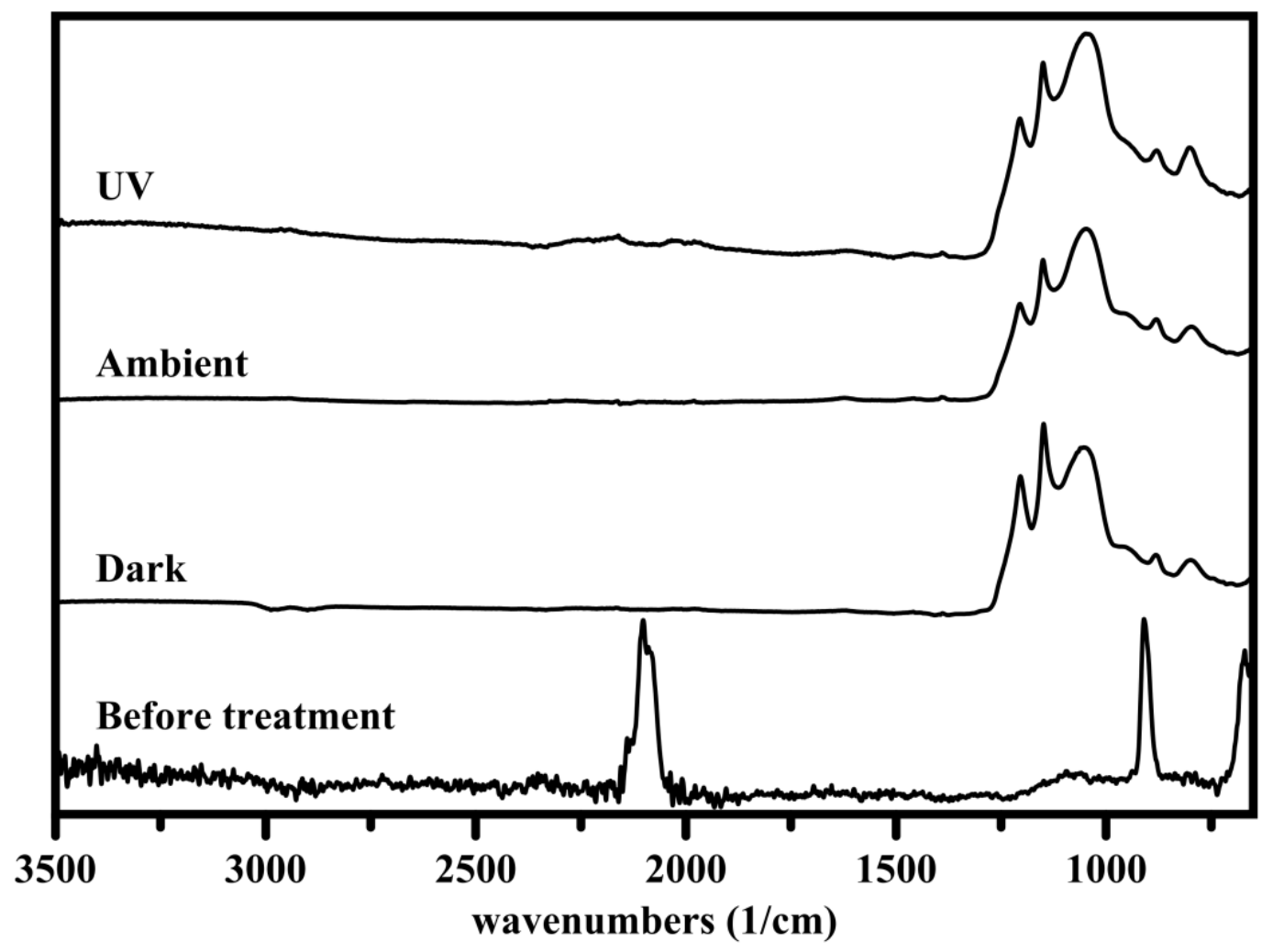

Figure 2.7: FTIR spectra for set of $1 \mathrm{mM}$ TEMPO treated samples. Traces (from bottom to top) are H-Si NPs prior to treatment, held in the dark, ambient lighting, and $365 \mathrm{~nm}$ irradiation.

large increases in the Si-O-Si stretching $\left(1080 \mathrm{~cm}^{-1}\right)$, Si-O-Si bending $\left(880 \mathrm{~cm}^{-1}\right)$, and $\mathrm{Si}-\mathrm{OH}$ (stretching $800 \mathrm{~cm}^{-1}$ ) modes. Additionally, there are two vibrational modes at $1210 \mathrm{~cm}^{-1}$ and $1150 \mathrm{~cm}^{-1}$ that are of unknown origin. The complete absence of $\mathrm{Si}-\mathrm{H}$ stretching after treatment with $1 \mathrm{mM}$ TEMPO indicates that the entire surface has been oxidized.

Samples treated with $5 \mathrm{mM}$ TEMPO showed nearly identical FTIR spectra as the $1 \mathrm{mM}$ TEMPO treatment. Figure 2.8 (following page) shows the FTIR spectra for samples treated with $5 \mathrm{mM}$ TEMPO. For all three lighting conditions, 


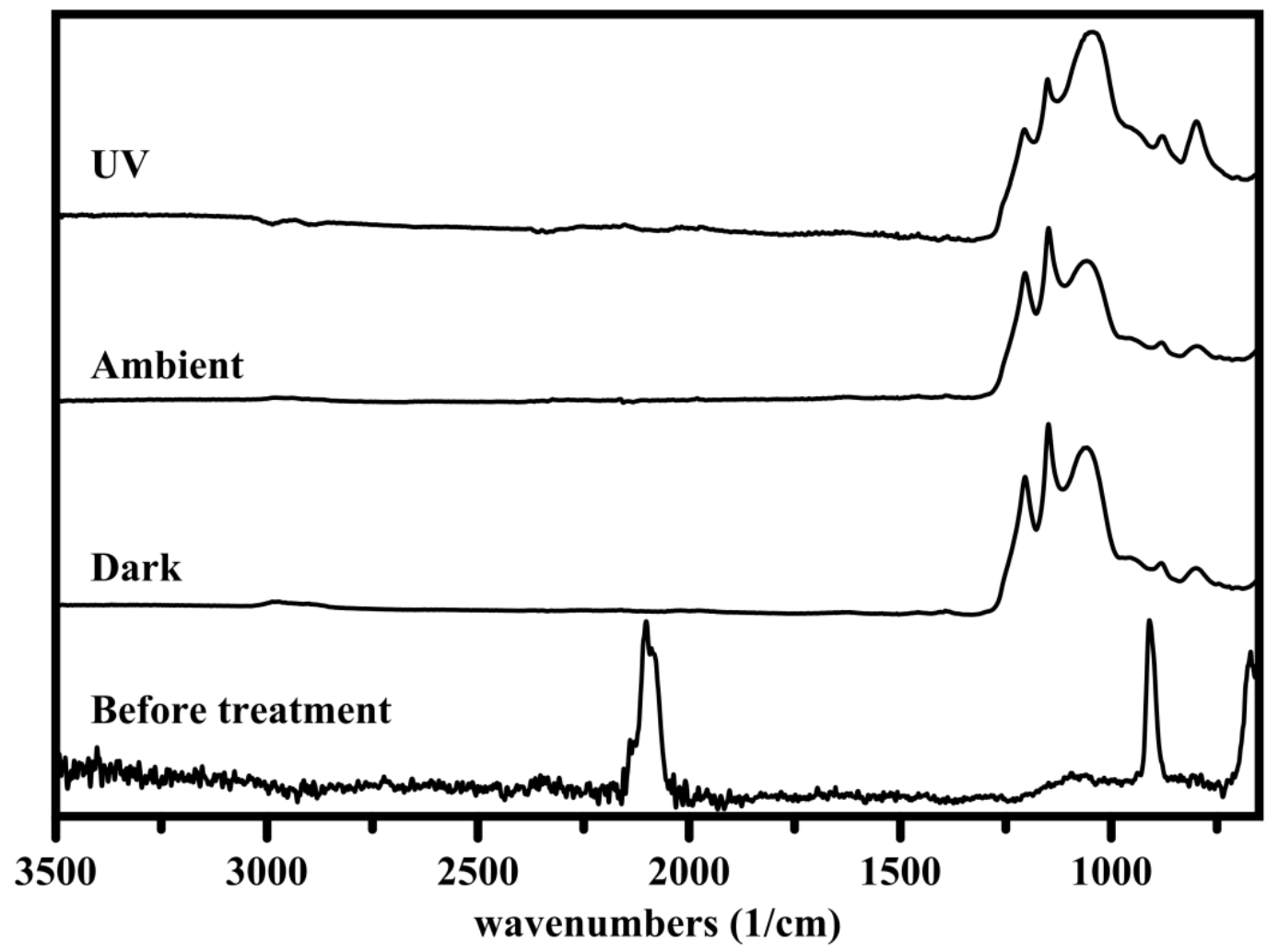

Figure 2.8: FTIR spectra for set of $5 \mathrm{mM}$ TEMPO treated samples. Traces (from bottom to top) are H-Si NPs prior to treatment, held in the dark, ambient lighting, and $365 \mathrm{~nm}$ irradiation.

the $1 \mathrm{mM}$ and $5 \mathrm{mM}$ TEMPO treatment eliminated the $\mathrm{Si}-\mathrm{H}$ vibrations and increased Si-O related vibrations and additional strong vibrations are observed at $1210 \mathrm{~cm}^{-1}$ and $1150 \mathrm{~cm}^{-1}$ that are from an unknown origin.

The ratio of the sharp $1210 \mathrm{~cm}^{-1}$ and $1150 \mathrm{~cm}^{-1}$ peaks to the broad 1080 $\mathrm{cm}^{-1} \mathrm{Si}-\mathrm{O}-\mathrm{Si}$ seems to be dependent on lighting conditions for both $1 \mathrm{mM}$ and 5 $\mathrm{mM}$ TEMPO treatments (Figures 2.7 and 2.8). In both $1 \mathrm{mM}$ and $5 \mathrm{mM}$ treatments the ratio of the sharp $1210 \mathrm{~cm}^{-1}$ and $1150 \mathrm{~cm}^{-1}$ features to the broad $1080 \mathrm{~cm}^{-1}$ peak decreases in the order of dark, ambient lighting, and then the UV treatment. 
It is possible that the lower input of energy has allowed for a more thermodynamically stable surface than a kinetically favored, higher energy UV treatment, where a more rapid oxidation of the surface occurs. The Si-O-Si stretching region of $1200 \mathrm{~cm}^{-1}$ to $1000 \mathrm{~cm}^{-1}$ is usually observed as a strong broad peak at $1080 \mathrm{~cm}^{-1}$ or so with one or two shoulders at increasing energy. The broadness of these peaks are mostly due to the disordered nature of the Si-O-Si network and it is possible that the sharper peaks indicate a much more ordered structure. ${ }^{62,67-69}$

\subsubsection{XPS measurements before and after treatment}

XPS measurements were taken before and after the temporal PL study for all samples, in order to further examine compositional changes observed by FTIR
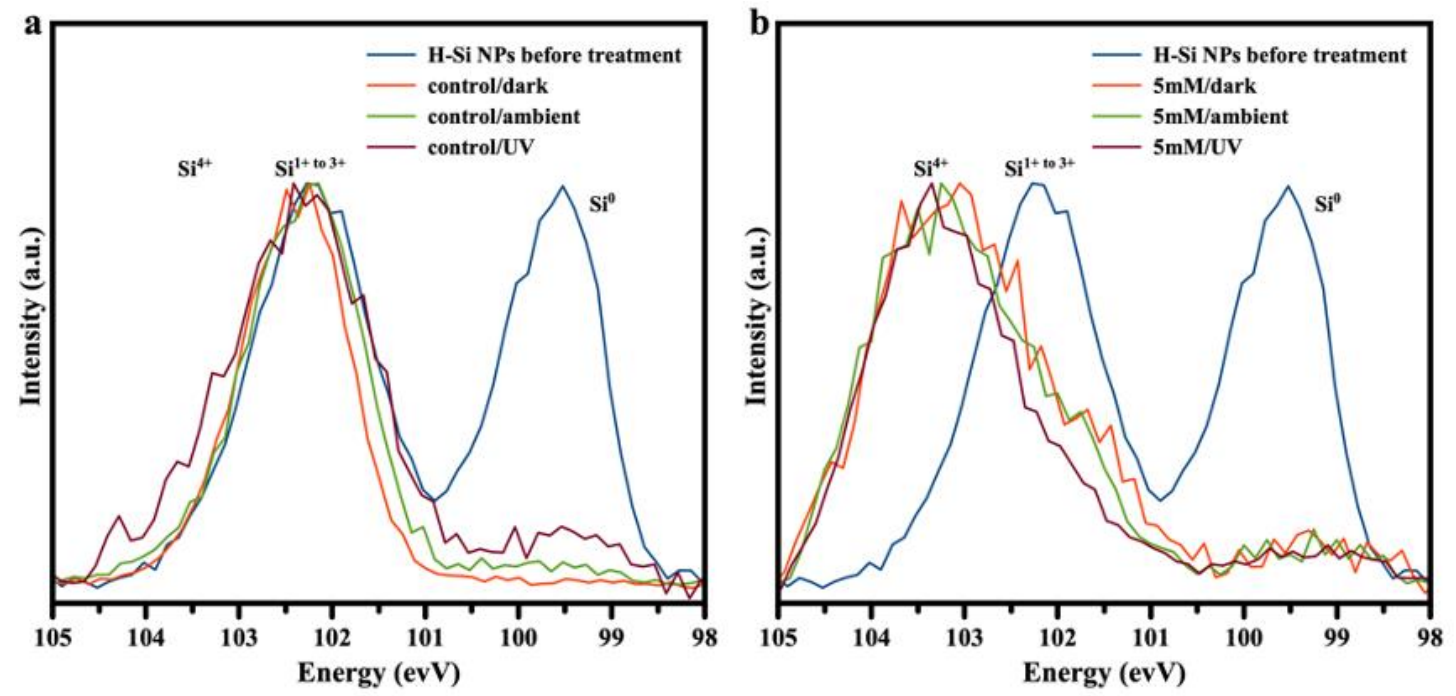

Figure 2.9: XPS binding energies in the $\mathrm{Si}(2 \mathrm{p})$ region before and after treatments for the control sample (a) and for the 5mM TEMPO treatment (b). The blue trace on each is the initial H-Si NPs prior to treatment and is the same spectrum. 
as a function of the TEMPO/light treatments. In the XPS data analysis, the $\mathrm{Si}(2 p)$ region can be fit with components to analyze the extent of surface oxidation and is described in detail in the experimental section using Figure 2.1 as an example of the fitting analysis. The XPS spectrum of the H-Si NPs prior to treatment (dark blue line of Figures 2.9a and 2.9b) has two main peaks, one centered at $99.5 \mathrm{eV}$ and one centered at $102.2 \mathrm{eV}$. The lower binding energy at $99.5 \mathrm{eV}$ indicates a large amount of $\mathrm{Si}^{0}$ present at or near the surface of the particles, while the peak $102.2 \mathrm{eV}$ is due to some oxidation of the surface $\mathrm{Si}$ atoms to a state of $1+$ to $3+$. There is a small contribution to the higher energy peak from a $\mathrm{Si}^{4+}$ but is mostly negligible (Figure 2.1). The control samples for all lighting conditions resulted in an almost complete reduction in the $\mathrm{Si}^{0}$ signal, however there is little shifting of the oxidation peak at $102 \mathrm{eV}$ to higher energy (Figure 2.9a). The 5mM TEMPO treatment, on the other hand, both showed both a decrease in $\mathrm{Si}^{0}$ signal as well as a shift in energy to $103.5 \mathrm{eV}$ for the oxidation peak (Figure 2.9b). The $1 \mathrm{mM}$ TEMPO treatment was identical to the $5 \mathrm{mM}$ TEMPO treatment and is not shown here.

Raman measurements were also taken to determine the overall crystallinity of the Si NPs and if the crystallinity changes over time, however the method of collection was insufficient for the samples and measurements were inconclusive due to the background being too strong and overwhelming the signal from the crystalline and amorphous silicon. 


\subsection{Discussion}

The initial goal of the experiment was to passivate surface radicals of the $\mathrm{H}-\mathrm{Si}$ NPs with the stable radical TEMPO, thus eliminating non-radiative recombination from the surface radical trap sites and improving the photoluminescence intensity from the quantum confined core. The results from the PL experiments in Figures 2.4 and 2.5 for the $1 \mathrm{mM}$ and $5 \mathrm{mM}$ TEMPO treatments show increased intensity from the core emission under all lighting conditions. However, that increase in intensity comes at the cost of significantly blue shifting the emission maximum wavelength by over $100 \mathrm{~nm}$, mostly due to surface oxidation.

The initial hypothesis of the experiment was that a radical coupling reaction would occur at the surface of the Si NP between the dangling bond and the TEMPO radical, but the experimental analysis shows a different story. FTIR analysis of the TEMPO treated samples (Figures 2.7 and 2.8) show that the TEMPO treatment under all lighting conditions have completely removed all $\mathrm{Si}-\mathrm{H}$ and only $\mathrm{Si}-\mathrm{O}$ type bonding is seen post treatment. Complete oxidation of the surface from the TEMPO treatments are also supported by the XPS data (Figure 2.9b). The shift from predominately $\mathrm{Si}^{0}$ and $\mathrm{Si}^{1+}$ to ${ }^{3+}$ in the initial condition to predominately $\mathrm{Si}^{4+}$ on the surface further supports a deeper penetration of the oxide layer by the TEMPO treatment. Although the control samples did show a reduction in the $\mathrm{Si}^{0}$ oxidation state, they had a mostly $\mathrm{Si}^{1+}$ to $3+$ oxidation state, indicating that the surface is only partially oxidized. 
As mentioned in the chapter 1, the effective mass approximation (equation 1.1) relates particle size to emission wavelength ${ }^{23}$ and has been demonstrated to be a good predictor for the emission from Si-NP with a diameter of $2.7 \mathrm{~nm}$ to 11.8 nm. ${ }^{53}$ Using the effective mass approximation, the emission wavelength of the quantum confined core before treatment $(662 \mathrm{~nm})$ would be attributed to a crystalline core diameter of $2.06 \mathrm{~nm}$. The largest energy shift (final lambda max of $520 \mathrm{~nm}$ ) was observed for the sample treated with $5 \mathrm{mM}$ TEMPO held in the dark. Again, using the effective mass approx. the new core size would be 1.60 $\mathrm{nm}$, a change of only $0.46 \mathrm{~nm}$. Since the nearest neighbor distance for silicon is $0.235 \mathrm{~nm}$, a completely oxidized surface could result in the large blue shift in emission that has been observed.

The reason for the complete oxidation of the surface is most likely due to the weak $\mathrm{N}-\mathrm{O}$ bond on the TEMPO radical. The $\mathrm{N}-\mathrm{O}$ bond energy is $2.01 \mathrm{eV}$, correlating to a photon wavelength of $617 \mathrm{~nm}$, and could be easily cleaved by both the ambient and UV lighting sources. For both TEMPO concentrations the increase in emission intensity occurs more rapidly under the UV treatment than ambient lighting alone, and even more slowly without any light source, supporting the breaking of this $\mathrm{N}-\mathrm{O}$ bond as a possible mechanism for the oxidation of the surface.

Complete oxidation of the surface from the TEMPO treatments are also supported by the XPS data (Figure 2.9b). The shift from predominately $\mathrm{Si}^{0}$ and $\mathrm{Si}^{1+}$ to $3+$ in the initial condition to predominately $\mathrm{Si}^{4+}$ on the surface further supports 
the deeper penetration of the oxide layer by the TEMPO treatment. Although the control samples did show a reduction in the $\mathrm{Si}^{0}$ oxidation state, they had a mostly $\mathrm{Si}^{1+}$ to $3+$ oxidation state, indicating that the surface is only partially oxidizing.

Although all samples were assumed to be kept in air and water free environments, FTIR indicates there was still some oxidation of the surface with no TEMPO present (figure 2.5) in the dark and ambient lighting conditions. This is most likely due to introduction of some oxygen when each aliquot was taken. The UV lighting condition, however, had complete oxidation of the surface with no remaining Si-H bonding present. It has previously been shown that continuous UV excitation of hydrogenated Si surfaces can result in an energy transfer to adsorbed $\mathrm{O}_{2}$, generating singlet $\mathrm{O}_{2}$ which can then react with the hydrogenated surface ${ }^{70,71}$ and this could be the reason for the accelerated oxidation of the surface under the UV lighting condition.

\subsection{Conclusions}

The TEMPO treatment observed in this experiment did increase luminescence intensity of the suspended Si NPs but did so with a large amount of oxidation to the surface. The extent of the oxidation was so great that the intrinsic core emission wavelength blue shifted by over $100 \mathrm{~nm}$. The oxidation of the $\mathrm{Si}$ NPs in the control samples indicate that the system is not in fact oxygen free. It is possible that trace oxygen in the solvents was the cause of this oxidation, but it is also possible that there is interstitial oxygen in either the amorphous or crystalline 
portions of the Si NPs and that over time these defects could be annealed out of the particles. The control experiments, on the other hand, did show that some oxidation could be used to increase the emission intensity of the suspended $\mathrm{Si}$ NPs and that having control over this process could result in a more gradual shift in emission wavelength. 
Chapter 3: Benzoyl peroxide as a surface passivating agent to improve photoluminescence intensity and colloidal stability of silicon nanoparticles

\subsection{Introduction}

The results of the TEMPO surface treatment in Chapter 2 told us that TEMPO is oxidizing the surface of the particles and while this oxidation does increase the PL intensity of the emission from the quantum confined core, it also significantly reduces the core size by oxidizing further into the core (not just surface passivating) that results in a blue shift of the $\lambda_{\max }$ to below $600 \mathrm{~nm}$. It was also evident that the control samples, while not exposed to TEMPO, did have some oxidation and the PL intensity increased with less blue shift in $\lambda \max$ than for the samples exposed to TEMPO. It was concluded that the surface oxidation of the H-Si NPs contributed to the increased emission intensity and that the only shifting in emission maximum wavelength was due to core shrinkage.

From these results, we hypothesized that the use of a mild oxidizing agent could control the extent of surface oxidation, thus increasing the PL intensity without significant blue shifting of the $\lambda_{\max }$ emission by core shrinkage. To test this we will subject the H-Si NPs to numerous oxidizing agents to identify the best combination of PL intensity increase and $\lambda_{\max }$ stability. The best treatment procedure will then be identified by subjecting the H-Si NPs to different concentrations of the best performing oxidant and different lighting conditions that 
were also shown to have an impact on the oxidation process from the TEMPO results.

\subsection{Experimental}

\subsubsection{Materials}

Ethanol (200 proof, TCI), Acetone (ACS reagent grade), DMSO (ACS reagent grade), toluene (ACS reagent grade), and benzoyl peroxide (75wt\% with the remainder $\mathrm{H}_{2} \mathrm{O}$ ) were purchased from Sigma Aldrich. Molecular sieves were used to dry all materials and were then sparged with Ar for 90 minutes to remove as much $\mathrm{O}_{2}$ as possible. All solutions were then transferred to a $\mathrm{N}_{2}$ filled glovebox $\left(<0.1 \mathrm{ppm} \mathrm{H}_{2} \mathrm{O}\right.$ and $\left.<0.1 \mathrm{ppm} \mathrm{O}_{2}\right)$ for further use.

\subsubsection{Synthesis of H-SiNPs}

H-Si NP suspensions were made by a literature procedure. ${ }^{14,62}$ Briefly, $\mathrm{HSiCl}_{3}$ was hydrolyzed and condensed upon the addition of $\mathrm{H}_{2} \mathrm{O}$ to make an $\left[\mathrm{HSiO}_{1.5}\right]_{\mathrm{n}}$ polymer. The polymer was then annealed at $1100^{\circ} \mathrm{C}$ for $10 \mathrm{hr}$ under flowing $\mathrm{N}_{2}$ to produce $\mathrm{Si} \mathrm{NPs}$ in an $\mathrm{SiO}_{2}$ matrix. The Si NPs were both liberated from the $\mathrm{SiO}_{2}$ matrix and hydride passivated using an aqueous $\mathrm{HF}$ etch. The $\mathrm{H}-\mathrm{Si}$ NPs were then phase transferred into toluene. The H-Si NP suspension was centrifuged at $2000 \mathrm{rpm}$ for 10 minutes in glass centrifuge tubes. The supernatant was then discarded, and the solid pellet was then re-suspended in dry toluene. This was repeated three times to ensure removal of all $\mathrm{HF}$, ethanol, and $\mathrm{H}_{2} \mathrm{O}$ that 
could still be present from the etching procedure. The final H-Si NPs were suspended in dry, degassed toluene at a concentration of approx. $0.1 \mathrm{mg} / \mathrm{mL}$.

\subsubsection{Oxidant Survey}

The oxidants chosen for the survey study were ethanol, acetone, DMSO, and benzoyl peroxide (BPO). Solutions of ethanol, acetone, DMSO, and BPO were made at a concentration of $10 \mathrm{mM}$ in toluene. Solutions were also made at a 1:1 volume ratio of ethanol, acetone, or DMSO with toluene. To observe changes to the PL caused by exposure to the atmosphere, dry and $\mathrm{O}_{2}$ free toluene was intentionally contaminated with either $\mathrm{H}_{2} \mathrm{O}$ or $\mathrm{O}_{2}$ prior to mixing with $\mathrm{H}-\mathrm{Si}$ NPs suspensions. This was done by thoroughly mixing water with toluene and then allowing the organic and aqueous phases to separate. The organic phase was then extracted and air was removed via three freeze/pump/thaw cycles on the Schlenk line. $\mathrm{O}_{2}$ was added to dry toluene by first vigorously shaking the solution while open to the atmosphere, then allowed to sit over $4 \AA$ molecular sieves for two days to remove any residual water that was present in the air.

$5 \mathrm{~mL}$ of each solution was added to an air free scintillation vial, to which 5 $\mathrm{mL}$ of a stock H-Si NP suspension was added. All samples were then held in the dark under constant stirring, periodically checking for luminescence changes with a handheld UV light ( $365 \mathrm{~nm}$ ) until significant changes to either brightness or color were qualitatively observed. After 4 days (the time it took for significant change to 
occur for more than one sample) PL spectra for all conditions were measured and analyzed by using the fitting procedure described in Chapter 2 .

\subsubsection{Benzoyl peroxide surface treatment study}

Benzoyl peroxide (BPO) was added to dry and degassed toluene to make $5 \mathrm{~mL}$ solutions that were $20 \mathrm{mM}, 10 \mathrm{mM}$, and $2 \mathrm{mM}$ in BPO; a control sample (initially only dry, degassed toluene) with no BPO was also prepared and handled identically. To each of these samples, $5 \mathrm{~mL}$ of $\mathrm{H}$-Si NPs $(0.1 \mathrm{mg} / \mathrm{mL})$ in toluene was added, resulting in solutions having final BPO concentrations of $10 \mathrm{mM}, 5 \mathrm{mM}$, $1 \mathrm{mM}$, and $0 \mathrm{mM}$ (control) and equal concentrations of H-Si NPs. These samples containing $\mathrm{H}-\mathrm{Si}$ NPs and variable concentrations of BPO were then left under constant $365 \mathrm{~nm}$ radiation ( $14.4 \mathrm{~W}$ ) or kept in the dark for $24 \mathrm{hr}$ to observe changes to the PL.

\subsubsection{Photoluminescence measurements}

Steady state photoluminescence (PL) measurements were conducted on a Shimadzu 5301-RFPM spectrophotometer equipped with a Xe laser source. All measurements were conducted using $365 \mathrm{~nm}$ excitation. A $365 \mathrm{~nm}$ band pass filter was used on the source side to prevent the passage of the $730 \mathrm{~nm}$ light from the diffraction grating. A $450 \mathrm{~nm}$ long pass filter was used on the detector side to prevent shorter wavelength light from saturating the detector. The raw PL spectral curves were then simulated using peak fitting software (MagicPlot 2.7.2) to model 
the spectra. The fitting procedure is thoroughly discussed for representative spectra in the results section of Chapter 2 (page 22).

\subsubsection{FTIR and XPS analysis}

Sample preparation, instruments and instrument parameters used, and analysis methodology are all identical to the FTIR and XPS procedures detailed in Chapter 2 (pages 16-18).

\subsection{Results}

\subsubsection{Oxidant Survey}

An initial survey of chemical oxidants that have been shown to oxidize $\mathrm{Si}$ was conducted. The chemical oxidants included ethanol, ${ }^{15} \mathrm{DMSO},{ }^{72}$ acetone, air, ${ }^{73-77}$ water, and benzoyl peroxide (BPO). Of the oxidants surveyed, only BPO

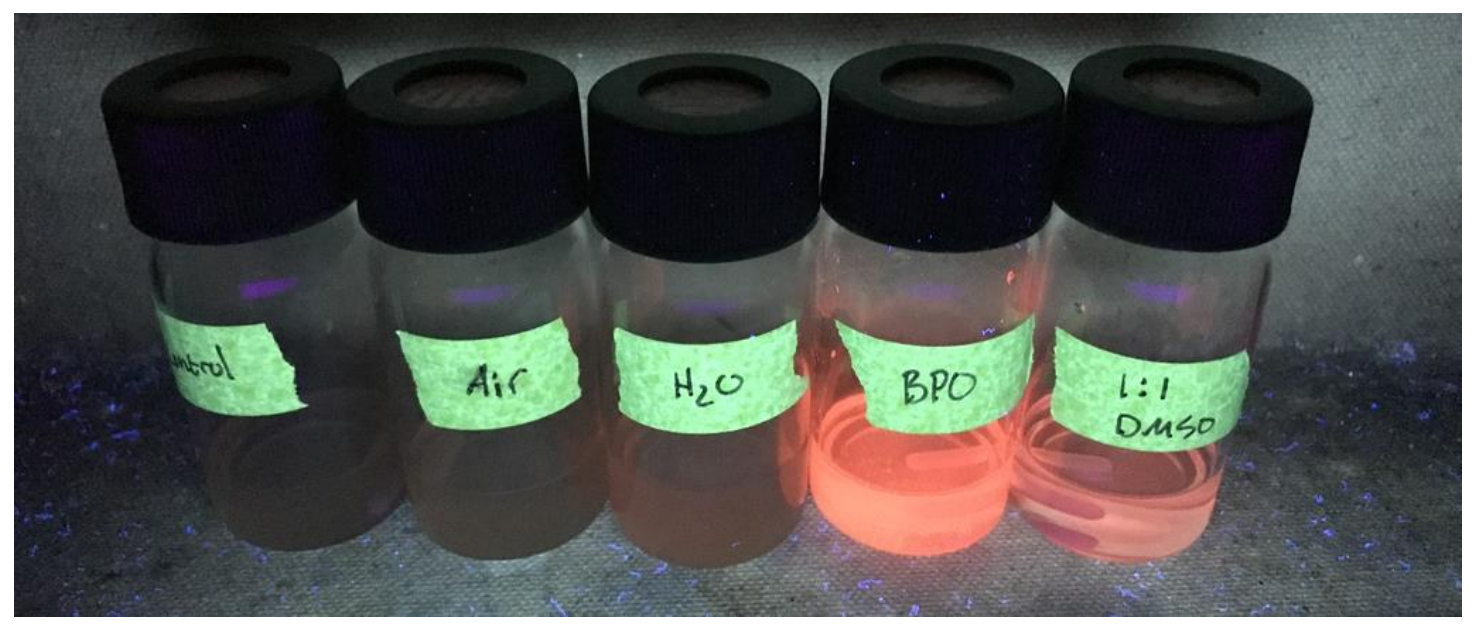

Figure 3.1: H-Si NPs in toluene after 4 days of exposure to the chemical oxidants under $365 \mathrm{~nm}$ irradiation. The oxidants being shown from left to right are the control sample, air, water, BPO, and the 1:1 DMSO concentration. 
and DMSO were observed to have any effect on increasing the photoluminescence intensity of the Si NPs core emissive state and are qualitatively shown in Figure 3.1. All other treatments completely quenched the core emission from the $\mathrm{H}-\mathrm{Si}$ NPs. The 1:1 DMSO to toluene treatment resulted in a slight increase in core emission intensity, however it also blue shifted the emission maximum wavelength from $687 \mathrm{~nm}$ to $602 \mathrm{~nm}$ (PL measurements not shown). The $10 \mathrm{mM}$ BPO treatment had a much larger increase in PL intensity from the core emission, with less significant blue shifting of the $\lambda_{\max }$ emission from $687 \mathrm{~nm}$ to $633 \mathrm{~nm}$ (PL measurements not shown).

\subsubsection{PL measurements from benzoyl peroxide treatment}

Using the results from the oxidant survey, we further investigated the effect of BPO addition on the PL peak position(s) and relative intensity of as a function of the BPO concentration. In the oxidant survey the samples were all held in the dark, however we expanded the study of BPO treatments to include the effect of lighting conditions on the extent of surface passivation. Since the peroxide bond in BPO could be homolytically cleaved from absorption of UV light, we hypothesized that treatment under UV lighting $(365 \mathrm{~nm})$ may either increase the extent of passivation or increase the reaction rate. We further examined possible composition (and/or size) differences underlying these changes using FTIR, XPS, and Raman spectroscopies. 
For the samples that were continuously irradiated using $365 \mathrm{~nm}$ light for 24 hours, PL measurements were taken and are shown in Figure 3.2. Prior to irradiation, the control sample is not highly luminescent; its emission spectrum shows a small peak at $575 \mathrm{~nm}$ and no distinguishable peak in the desired red region (600 $\mathrm{nm}$ to $700 \mathrm{~nm}$ ). After 24 hours of UV irradiation, the emission spectrum of the control sample with no BPO looks largely identical to the start. Conversely, the emission spectra of the H-Si NP samples treated with BPO displayed increased intensity, with discernable peaks at $575 \mathrm{~nm}$, and $650 \mathrm{~nm}$. Although the peak

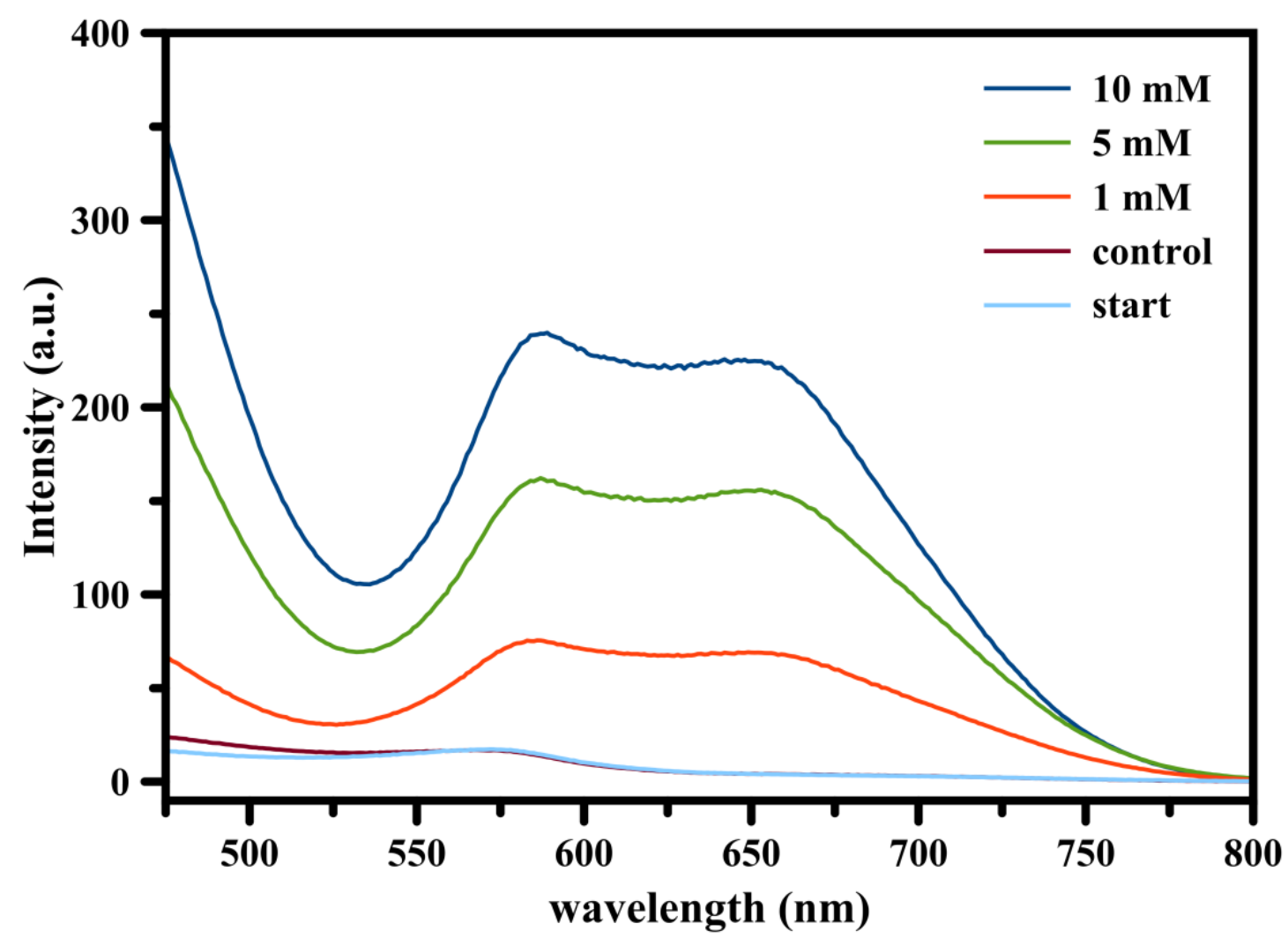

Figure 3.2: PL spectra (excited at $365 \mathrm{~nm}$ ) of $\mathrm{H}-\mathrm{Si}$ NPs in toluene with variable concentration of BPO, taken after 24 hours of continuous irradiation of the samples with $365 \mathrm{~nm}$ light. 
positions did not differ across the three samples with BPO concentrations in the 1$10 \mathrm{mM}$ range, the intensities of the $570 \mathrm{~nm}$ and $650 \mathrm{~nm}$ emissions increased with increasing BPO concentration. The most likely explanation for the increased intensity is due to better surface passivation of the Si NPs. The lack of blue shifting also indicates that the BPO does interact with the surface but does not oxidize the particles deeper into the core.

The BPO treated samples that were held in the dark did not show any change in intensity over the first 24 hours of treatment, thus the samples were kept in the dark in an air free environment under stirring for 24 days, after which the PL

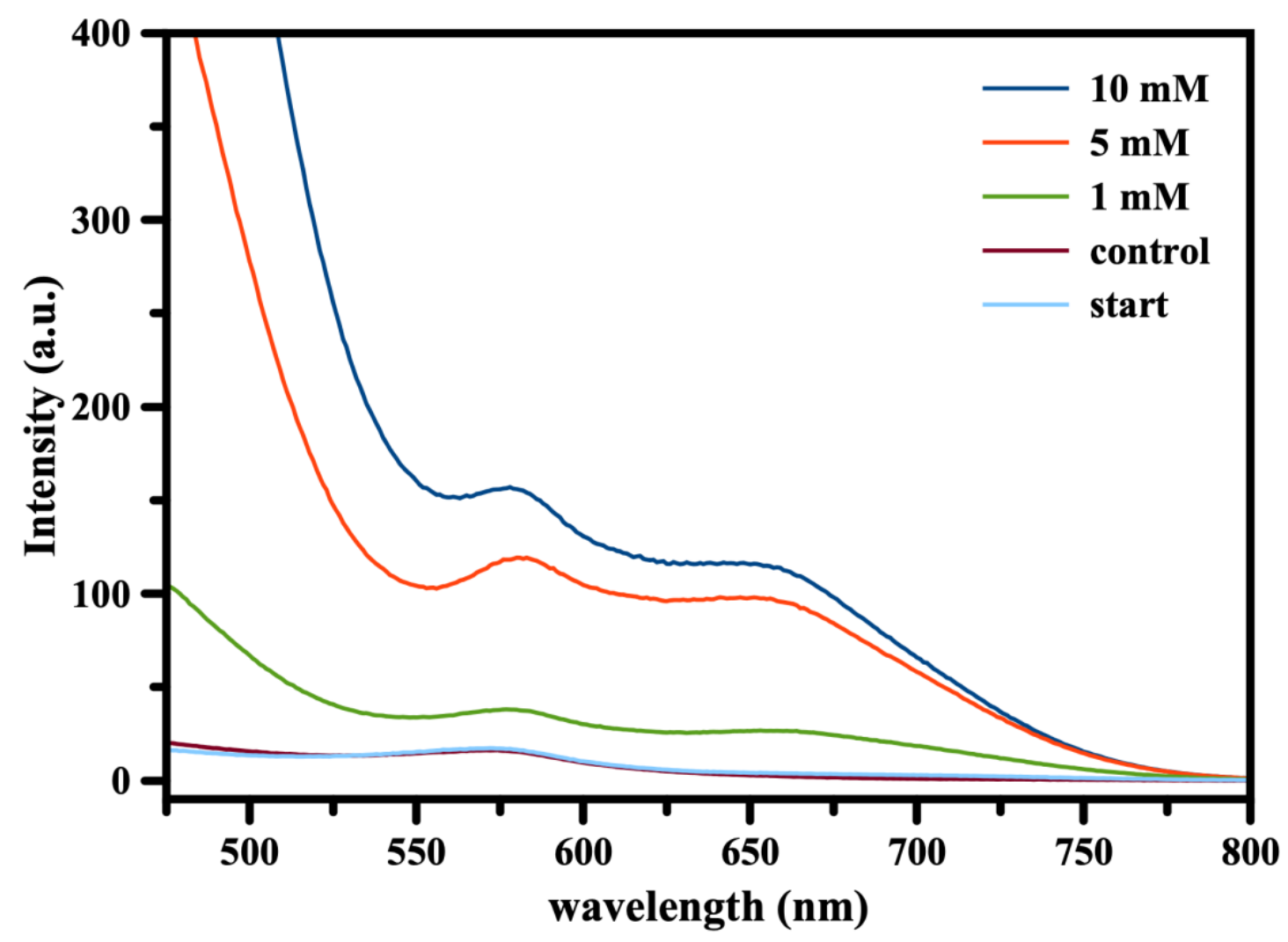

Figure 3.3: $\mathrm{PL}$ spectra (excited at $365 \mathrm{~nm}$ ) of $\mathrm{H}-\mathrm{Si}$ NPs in toluene with variable concentration of BPO, taken after 24 days of treatment in the dark. 
was measured again using a $450 \mathrm{~nm}$ long-pass filter and a $365 \mathrm{~nm}$ bandpass filter (Figure 3.3). The control H-Si NPs held in the dark with no BPO did not have any appreciable change in emission from the pre-treatment measurement over the 24 day period. There is gradual decrease in emission intensity from the $475 \mathrm{~nm}$ cut on and a small peak at $575 \mathrm{~nm}$ with no apparent peak in the 600 to $700 \mathrm{~nm}$ range. The $1 \mathrm{mM}$ treated sample showed a 4 fold increase from the pre-treated sample in the intensity of the cut on at $475 \mathrm{~nm}$ due to a blue emissive component, followed by a peak at $570 \mathrm{~nm}$ and a peak at $650 \mathrm{~nm}$. The $5 \mathrm{mM}$ and $10 \mathrm{mM}$ samples have much larger blue emissive components followed by peaks at $570 \mathrm{~nm}$ and $650 \mathrm{~nm}$. The integrated intensity of the $570 \mathrm{~nm}$ and $650 \mathrm{~nm}$ components increases with increasing BPO concentration.

The blue component for all three BPO treated samples was much larger than the red, presumably due to the blue emission of free BPO in toluene. To
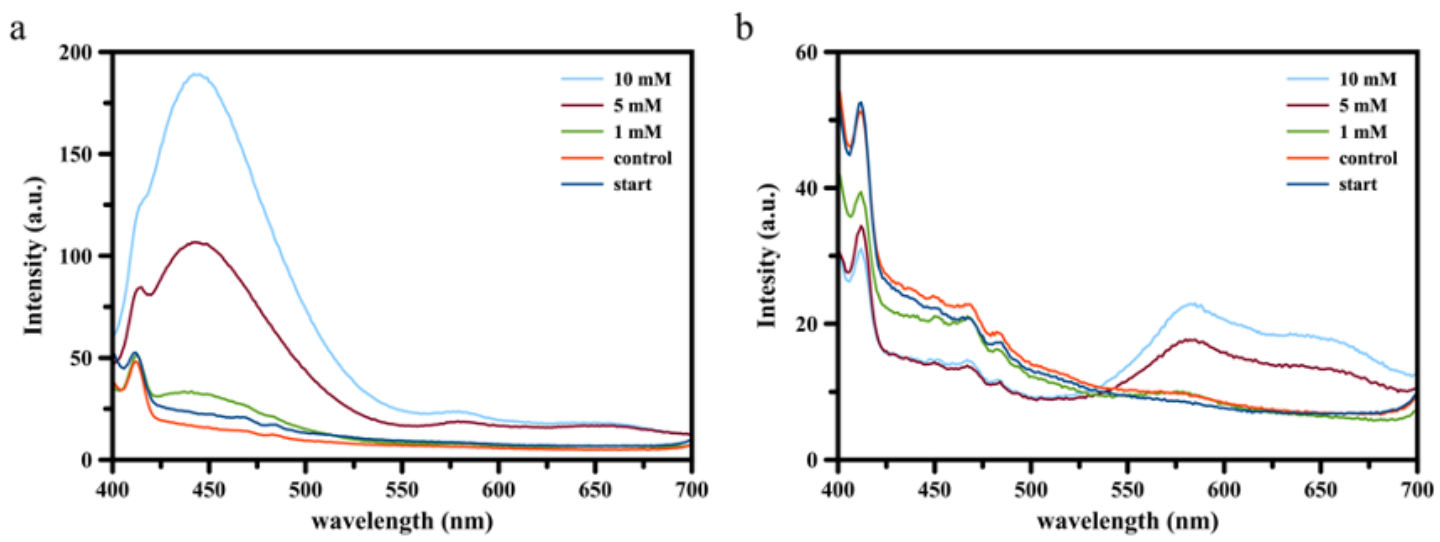

Figure 3.4: PL measurements before and after washing dark BPO treatment to remove blue emissive component. PL measurements without filters of (a) the BPO treatment in dark for 24 days and (b) the same particles after washing and re-dispersing them into toluene. 
demonstrate that the blue component was not due to the treated Si NPs, the suspensions were centrifuged, supernatant discarded, and the particles redispersed in dry toluene. This was done three times to remove any residual free BPO from the suspension. PL measurements were taken before and after the washing procedure without filters in place to show the full spectra and are presented in Figure 3.4. The before spectra (Figure 3.4a) is the extended spectra without filters from Figure 3.3. It should be noted that the final solution concentration of Si NPs post-washing is not identical for all samples measured due to losses during the washing procedure, thus the emission intensities for all of the washed samples (Figure 3.3b) cannot be directly compared to each other.

The pre-treatment sample and the control sample shows only a decrease in the emission with increasing wavelength and a small peak at $570 \mathrm{~nm}$, with no discernable emission in the $600 \mathrm{~nm}$ to $700 \mathrm{~nm}$ range (Figure 3.3a). All BPO treated samples have a peak at $475 \mathrm{~nm}$ that no longer looks like the scattering from the control and pre-treatment spectra in this region. The $5 \mathrm{mM}$ and $10 \mathrm{mM}$ samples also had $570 \mathrm{~nm}$ and $650 \mathrm{~nm}$ peaks that can be seen. The PL spectra after washing the treated Si NPs and re-suspending in toluene (Figure 3.3b), there is no longer any identifiable blue emissive component left in any of the suspensions.

The only observed intensity in the blue spectral region appears to be more consistent with scattering from aggregated particles. Although intensities cannot be directly compared from sample to sample after washing, the ratio of scattering intensity to red emission can be compared for each sample individually. The ratio 
of red emission to scattering intensity increases with increasing BPO treatment concentration, indicating better passivation of the particles. The suspensions post treatment and wash were visually less cloudy, and the decreased scattering in the PL spectra is an indication that Si NPs are less aggregated in suspension.

\subsubsection{FTIR measurements before and after BPO treatment}

The infrared absorption spectrum was measured for each sample condition before and after treatment to correlate changes to the PL over time with changes in surface structure. Figure 3.5 shows the IR absorption spectra for the H-Si NPs

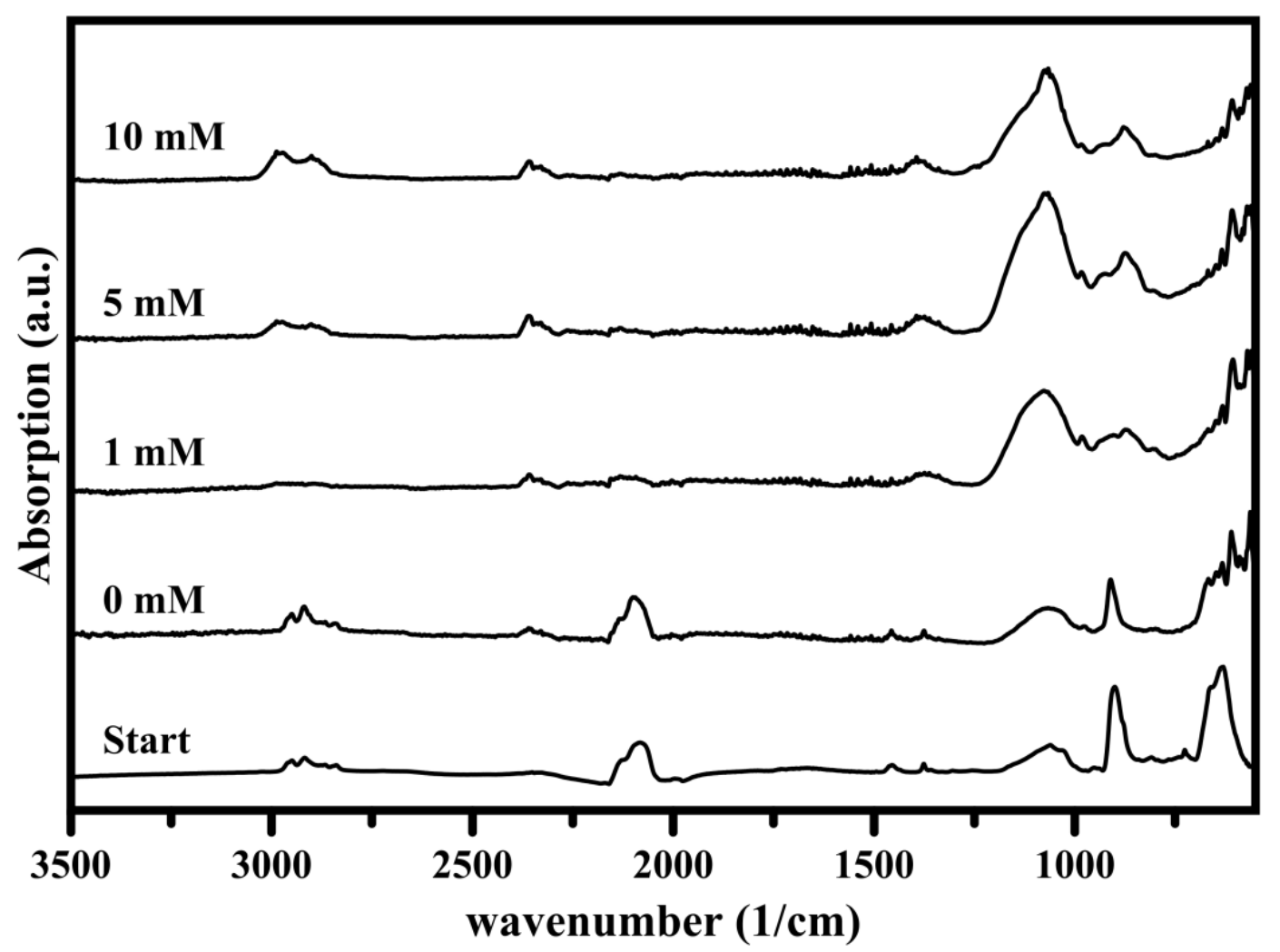

Figure 3.5: FTIR of BPO and UV treated samples. Traces are H-Si NPs prior to treatment and after exposure to $0,1 \mathrm{mM}, 5 \mathrm{mM}$, and $10 \mathrm{mM} \mathrm{BPO}$ and 365 $\mathrm{nm}$ light for 24 hours. 
pre-treatment and the $0 \mathrm{mM}, 1 \mathrm{mM}, 5 \mathrm{mM}$ and $10 \mathrm{mM}$ treatments under $365 \mathrm{~nm}$ irradiation for 24 hours. The pre-treatment sample has peaks at $2100 \mathrm{~cm}^{-1}$ and $910 \mathrm{~cm}^{-1}$ that are assigned to $\mathrm{Si}-\mathrm{H}$ stretching and scissoring respectively, and a broad feature centered at $1080 \mathrm{~cm}^{-1}$ assigned to Si-O-Si stretching. Peaks seen in the 2800 to $3000 \mathrm{~cm}^{-1}$ range, $1450 \mathrm{~cm}^{-1}$ and $1360 \mathrm{~cm}^{-1}$ can be assigned to possible $\mathrm{C}-\mathrm{H}$ and $\mathrm{C}-\mathrm{C}$ stretching from residual toluene that did not completely evaporate from the deposited Si NP film. The sample treated with only $365 \mathrm{~nm}$ light (no BPO) did not show significant change from the pre-treatment sample, with peaks at $2100 \mathrm{~cm}^{-1}$ and $910 \mathrm{~cm}^{-1}$ indicating Si-H surface bonds without increase to the Si-O-Si stretching at $1080 \mathrm{~cm}^{-1}$. The $1 \mathrm{mM}, 5 \mathrm{mM}$, and $10 \mathrm{mM}$ all show an elimination of the Si-H stretching and increase in the Si-O-Si stretching at 1080 $\mathrm{cm}^{-1}$. The doublet at $2250 \mathrm{~cm}^{-1}$ can be attributed to $\mathrm{CO}_{2}$ that adsorbed while the film was being deposited on the ATR crystal.

While the UV lighting condition did result in oxidation of the surface as evidenced by the Si-O-Si stretching increase and elimination of the Si-H stretching, there is no evidence of BPO bonding to the surface of the Si NPs. If BPO was chemically bound to the surface of the particles, there should be observed stretching modes at $1700 \mathrm{~cm}^{-1}$ (carbonyl), and $1600 \mathrm{~cm}^{-1}$ (aromatic). While there appears to be some features in the 2800 to $3000 \mathrm{~cm}^{-1}$ and $1300 \mathrm{~cm}^{-1}$ range that could indicate the presence of BPO on the surface, there is no identifiable carbonyl stretch in any of the spectra. The IR absorption spectra for the BPO treated samples that were held in the dark for 24 days (Figure 3.6) showed similar trends 
to that of the UV treated samples. The H-Si NPs held in the dark with no BPO had no significant change from the pre-treatment sample. The apparent increase in Si$\mathrm{H}$ intensity at $2100 \mathrm{~cm}^{-1}$ and $910 \mathrm{~cm}^{-1}$ is coupled with an increase in $\mathrm{Si}-\mathrm{O}-\mathrm{Si}$ stretching intensity at $1000-1200 \mathrm{~cm}^{-1}$ but the ratio of the intensities of $\mathrm{Si}-\mathrm{H}$ to Si$\mathrm{O}-\mathrm{Si}$ is similar to the starting material and the increase in intensity is due to a thicker film being measured. The $1 \mathrm{mM}$ sample maintains some $\mathrm{Si}-\mathrm{H}$ stretching at $2100 \mathrm{~cm}^{-1}$ and an increase in the Si-O-Si stretching at $1000-1200 \mathrm{~cm}^{-1}$. The $5 \mathrm{mM}$ treated sample has a small Si-H stretching peak at $2100 \mathrm{~cm}^{-1}$ and increased Si-O-

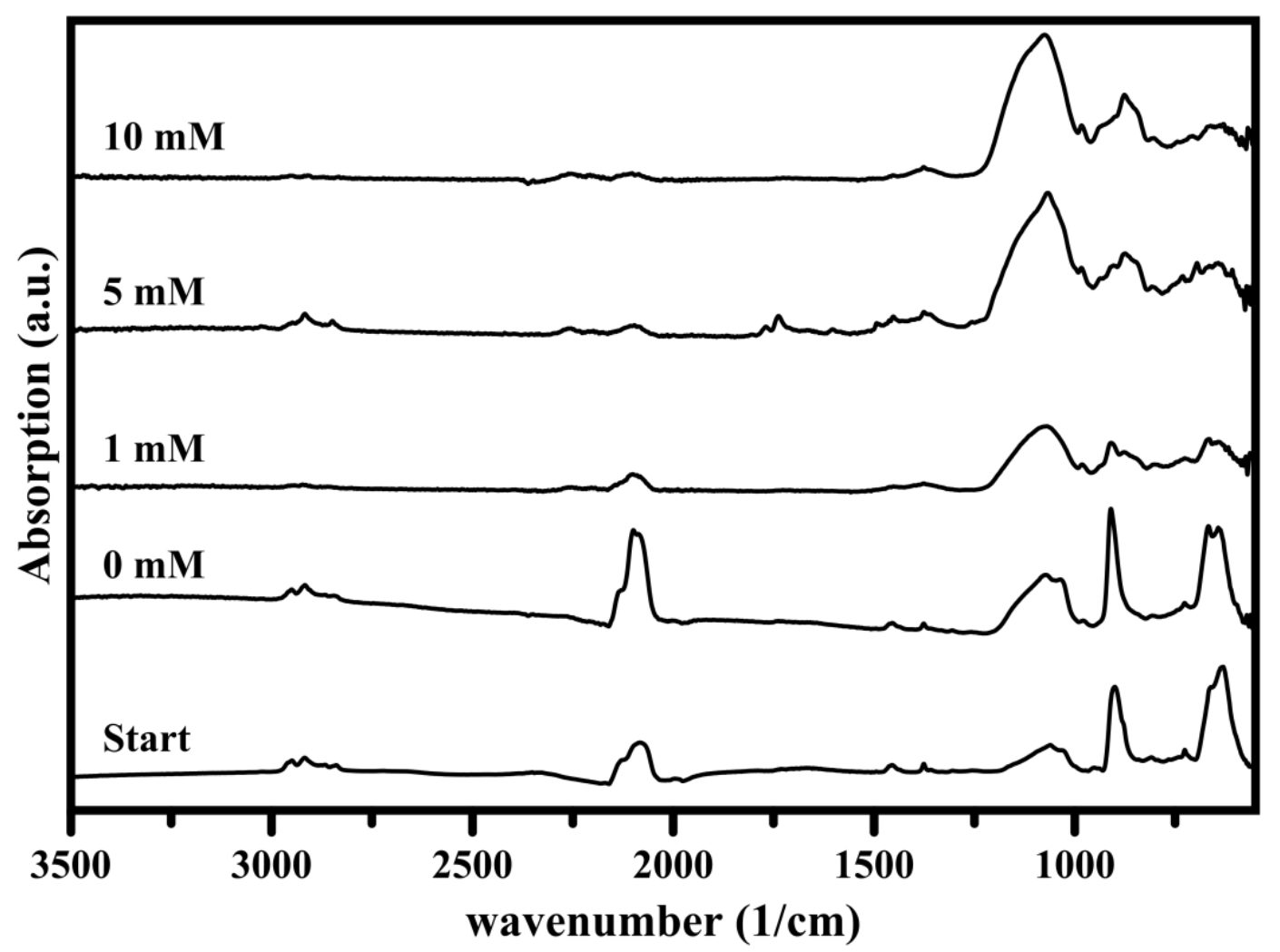

Figure 3.6: FTIR of BPO treated samples in the dark. Traces are H-Si NPs prior to treatment and after exposure to $0,1 \mathrm{mM}, 5 \mathrm{mM}$, and $10 \mathrm{mM}$ BPO and held in the dark for 24 days. 
Si stretching at $1000-1200 \mathrm{~cm}^{-1}$, but also has $\mathrm{C}-\mathrm{H}$ stretching features at 2800 to $3000 \mathrm{~cm}^{-1}$ and carbonyl stretching at $1740 \mathrm{~cm}^{-1}$ that could be due to residual BPO still in solution after the washing procedure. The $10 \mathrm{mM}$ treated sample did not show the carbonyl stretching at $1740 \mathrm{~cm}^{-1}$ and only Si-O-Si stretching at 1000 $1200 \mathrm{~cm}^{-1}$ is observed.

Similar to the UV treated sample, the intensity of the Si-O-Si stretching band increased with increasing concentration of BPO, coinciding with a decrease in intensity of the Si-H stretching with increasing BPO concentration. This suggests the particle surface is being oxidized by the BPO, however the BPO is not remaining on the surface of the particles. 


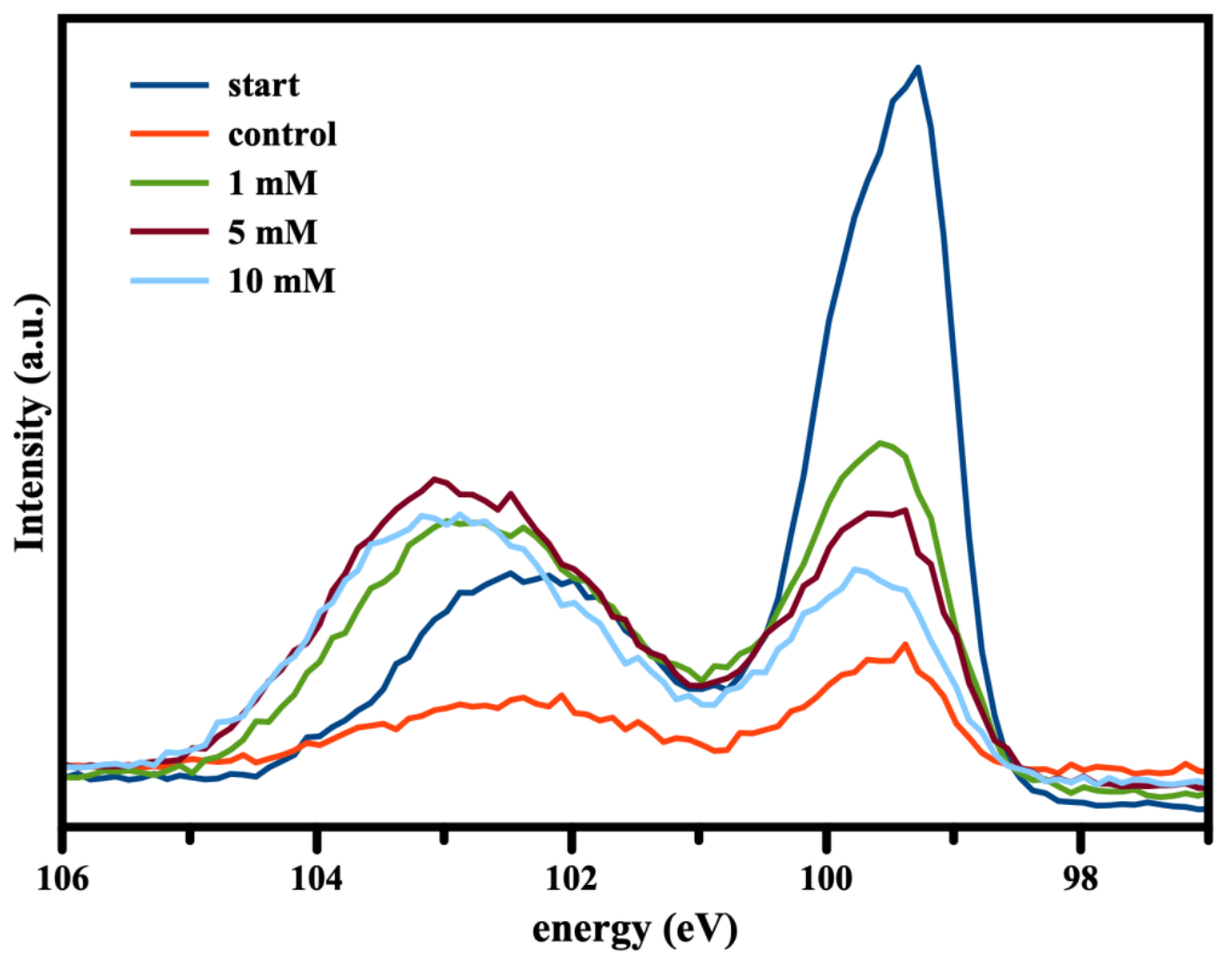

Figure 3.7: XPS spectra of the BPO and UV treated Si NPs. The Si $2 p$ region is shown before treatment and following BPO surface treatment under UV irradiation for 24 hours. The concentrations of the BPO in solution are indicated in the legend.

\subsubsection{XPS measurements before and after treatment}

To observed changes to the oxidation state of the surface of the Si NPs pre and post treatment, XPS measurements were conducted and the Si $2 p$ region was observed. It should be noted that the measured intensity from the sample is proportional to the amount of sample present, however the ratio of peaks within an individual spectrum is independent of sample mass. Figure 3.7 shows the Si $2 p$ peak for the samples treated with BPO and held under $365 \mathrm{~nm}$ irradiation for 24 
hours. The pre-treatment sample has a strong asymmetric peak at $99.5 \mathrm{eV}$ that is the doublet generated from Si $2 \mathrm{p}_{3 / 2}$ and $\mathrm{Si} 2 \mathrm{p}_{1 / 2}$ splitting from the $\mathrm{Si}^{0}$ oxidation state. There is also a smaller broad peak centered at $102.1 \mathrm{eV}$ that is due to $\mathrm{Si}$ that has been oxidized (oxidation state of +1 to +4 ). The sample treated with only $365 \mathrm{~nm}$ light and no BPO showed a decrease in intensity for the peak at $99.5 \mathrm{eV}$ and slight shift to $102.3 \mathrm{eV}$ of the oxidized Si peak, indicating increased oxidation state. The $1 \mathrm{mM}$ BPO treated sample showed an increase in the ratio of oxidized to unoxidized silicon and a further shift to $102.5 \mathrm{eV}$ of the oxidized peak. The 5 $\mathrm{mM}$ and $10 \mathrm{mM}$ BPO treated samples both had further increases to the ratio of oxidized to unoxidized Si with both having the oxidized Si peak centered at 102.8 eV.

XPS measurements of the BPO treatment conducted in the dark over 24 days are presented in Figure 3.8. The H-Si NPs held in the dark for 24 days with no BPO showed no change to the unoxidized Si peak at $99.5 \mathrm{eV}$ and a decrease in the oxidized Si peak and shift to $101.8 \mathrm{eV}$, a shift to lower energy relative to the pre-treatment sample indicating less oxidation. The BPO treated samples showed an increase in the oxidized peak to unoxidized peak ratio that was proportional to the BPO concentration. The oxidized Si peak for the $1 \mathrm{mM}, 5 \mathrm{mM}$, and $10 \mathrm{mM}$ samples also shifted to $102.3 \mathrm{eV}, 102.6 \mathrm{eV}$, and $102.8 \mathrm{eV}$ respectively. 


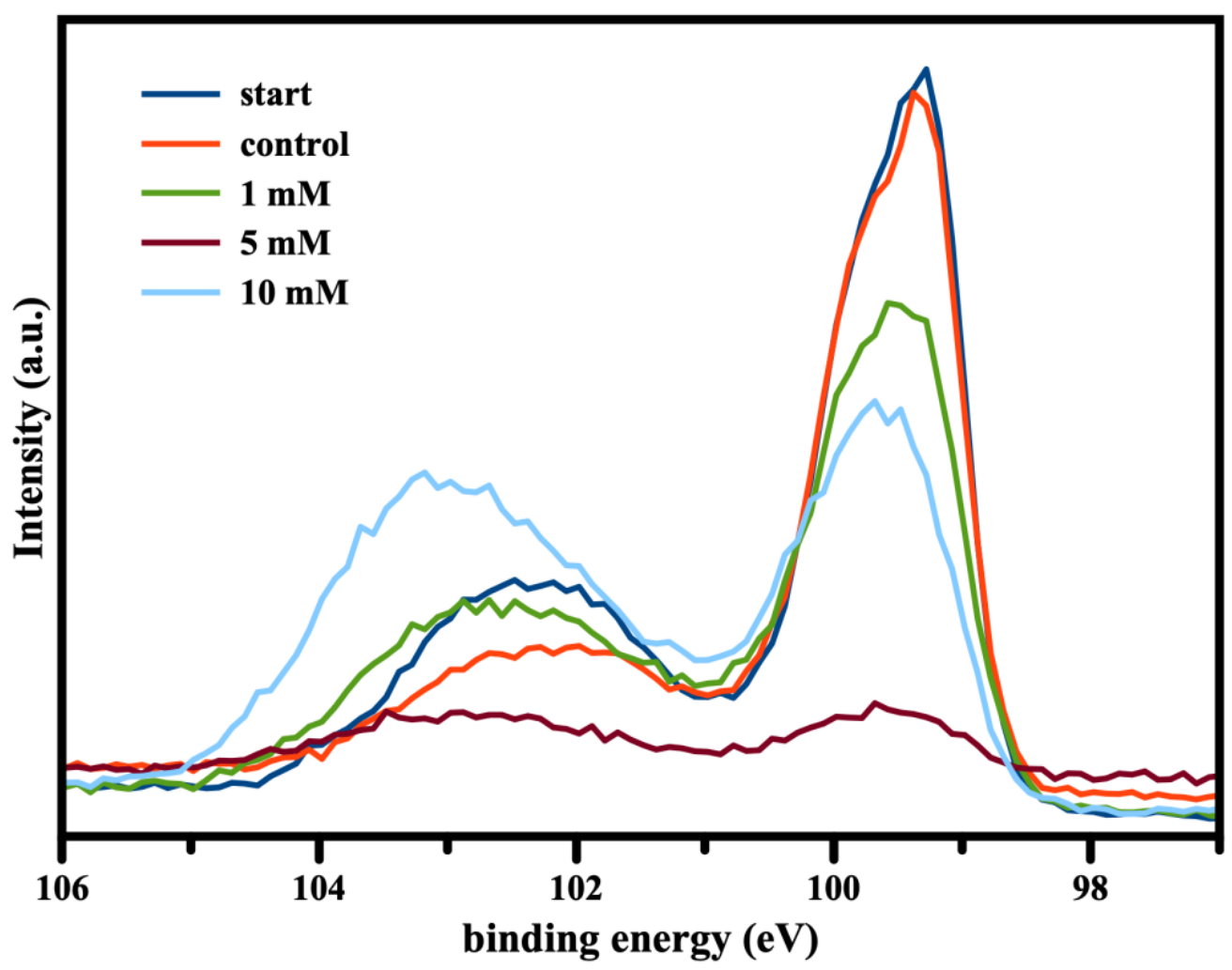

Figure 3.8: XPS spectra of the BPO treated Si NPs held in the dark. The Si $2 p$ region is shown before treatment and following BPO surface treatment in the dark for 24 days. The concentrations of the BPO in solution are indicated in the legend.

The broad oxidation peak represents multiple oxidation states and some reports have attempted to model each oxidation state with its own Gaussian curve. ${ }^{40}$ To model the oxidation on the surface of the H-Si NPs we chose to follow a procedure that uses 2 curves to represent oxidized silicon. ${ }^{63,64}$ One of these curves represents silicon in the +4 state (as in $\mathrm{SiO}_{2}$ ) that should be centered between 103 and $103.5 \mathrm{eV}$ while the other represents $\mathrm{Si}$ in the $1+$ to $3+$ oxidation state centered between 101 to $102.5 \mathrm{eV}$. The modeling of these peaks is demonstrated in Figure 


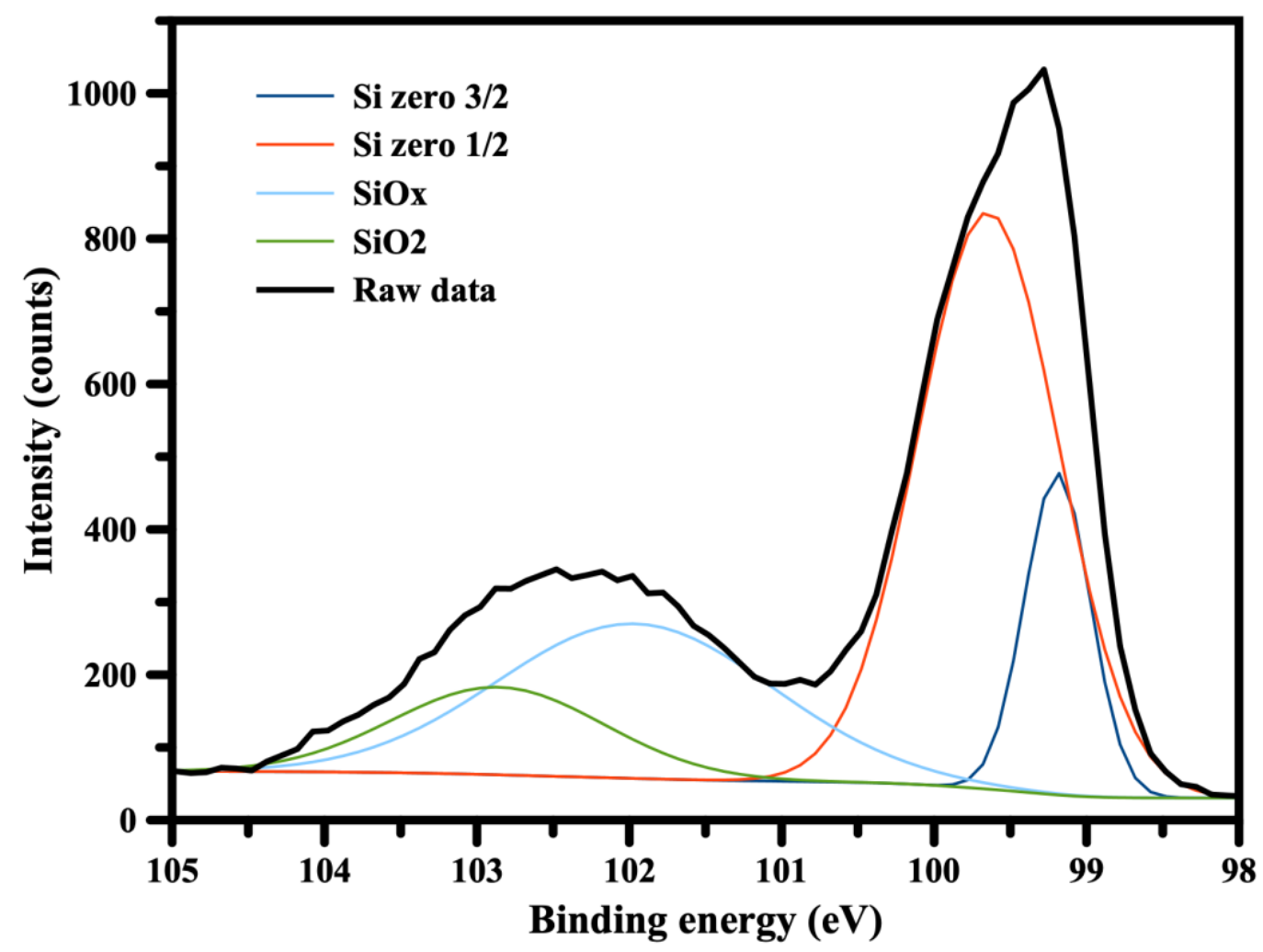

Figure 3.9: XPS of starting material showing the modeling procedure used. Four curves represent the two $\mathrm{Si}^{0}$ oxidation states (dark blue and red), an $\mathrm{Si}^{1+}$ to ${ }^{3+}$ oxidation state (light blue), and a $\mathrm{Si}^{4+}$ oxidation state.

3.9 where the $\mathrm{Si}^{0}$ doublet is shown and two peaks representing $\mathrm{Si}^{4+}$ and $\mathrm{Si}^{1+}$ to ${ }^{3+}$ are centered at $103 \mathrm{eV}$ and $102 \mathrm{eV}$ respectively. From the fitting results the integrated intensities of all components can be calculated and the percent contribution to the overall intensity of the Si $2 p$ spectrum can be determined. The simulated data in Figure 2.16 for the pre-treatment sample resulted in $61 \%$ with an oxidation state of zero, $25 \%$ with an oxidation state between $1+$ and $3+$, and $14 \%$ with an oxidation state of $4+$. 

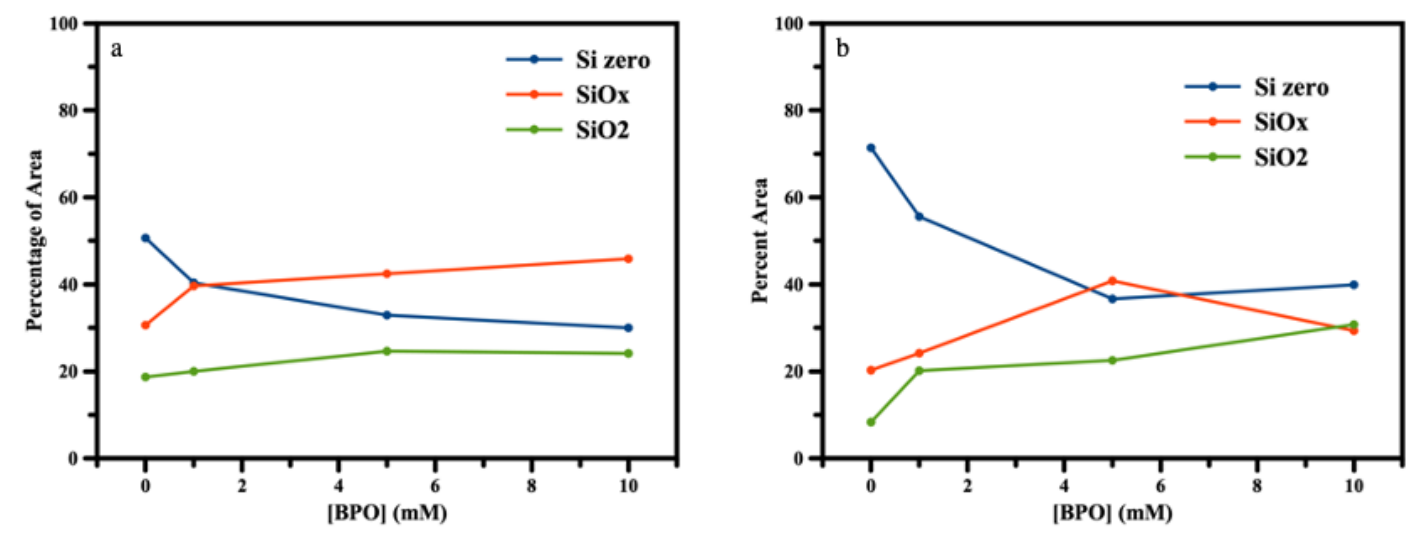

Figure 3.10: Percentage of intensity vs BPO concentration for each oxidation state from the simulated XPS data for the BPO treated samples held under 365 $n m$ irradiation (a) and in the dark (b).

All raw XPS data was similarly simulated and the percent contribution from each oxidation state was plotted against BPO concentration (Figure 3.10). For the samples held under $365 \mathrm{~nm}$ irradiation (Figure 3.10a), the $\mathrm{Si}^{0}$ peak percentage decreased from its initial value of $61 \%$ (not shown) to $51 \%$ for the $0 \mathrm{mM}$ treatment, $40 \%$ for the $1 \mathrm{mM}$ treatment, $33 \%$ for the $5 \mathrm{mM}$ treatment, and $30 \%$ for the $10 \mathrm{mM}$ treatment. The partially oxidized peak increase from an initial value of $25 \%$ (not shown) to $31 \%$ for the $0 \mathrm{mM}$ treatment, $40 \%$ for the $1 \mathrm{mM}$ treatment, $42 \%$ for the $5 \mathrm{mM}$ treatment, and $46 \%$ for the $10 \mathrm{mM}$ treatment. The $\mathrm{Si}^{4+}$ peak increased from its initial value of $14 \%$ (not shown) to $19 \%, 20 \%, 25 \%$, and $24 \%$ for the $0 \mathrm{mM}, 1$ $\mathrm{mM}, 5 \mathrm{mM}$, and $10 \mathrm{mM}$ treatments respectively. Overall, for the UV irradiated samples, the amount of elemental Si decreased while oxidized Si increased, consistent with the FT-IR results. 
The samples treated with BPO but held in the dark for 24 days (Figure $3.10 \mathrm{~b})$ showed an increase in the $\mathrm{Si}^{0}$ peak percentage from $61 \%$ for the pretreatment sample (not shown) to $71 \%$ for the sample with no BPO, followed by a decrease to $56 \%$ for the $1 \mathrm{mM}$ BPO treatment, $37 \%$ for the $5 \mathrm{mM}$ treatment, and $40 \%$ for the $10 \mathrm{mM}$ treatment. The partially oxidized Si peak percentage decreased from $25 \%$ initially (not shown) to $20 \%$ for the $0 \mathrm{mM}$ treatment, followed by an increase to $24 \%, 41 \%$, and $39 \%$ for the $1 \mathrm{mM}, 5 \mathrm{mM}$, and $10 \mathrm{mM}$ treatments respectively. The $\mathrm{Si}^{4+}$ peak decreased from the initial $14 \%$ (not shown) to $8 \%$ for the $0 \mathrm{mM}$ treatment, then increased to $20 \%, 23 \%$, and $31 \%$ for the $1 \mathrm{mM}, 5 \mathrm{mM}$, and $10 \mathrm{mM}$ treatments respectively.

Overall, BPO treatment resulted in a decrease in $\mathrm{Si}^{0}$ present in the samples and an increase in the oxidized $\mathrm{Si}$ in the samples and the extent of this effect is directly proportional to the concentration of BPO in the suspension.

\subsection{Discussion}

The overall goal of this project was to identify a novel surface treatment for the produce Si NPs with improved luminescence intensity without substantial change to emission wavelength. The PL measurements for both lighting conditions show that an increase in the BPO concentration increases the PL intensity attributed to the crystalline core. The maximum emission wavelength was also shown to stop at $650 \mathrm{~nm}$ for all conditions observed. Due to the very poor emission in the red region of the initial H-Si NPs prior to treatment, there was no 
determination of the starting emission wavelength. All previous experiments that were conducted using the same or similar parameters have yielded H-Si NPs that have a maximum emissive wavelength between approx. $660 \mathrm{~nm}$ to $690 \mathrm{~nm}$. The most likely cause of the dark state for our H-Si NPs before treatment is due to significant aggregation. ${ }^{77-79}$ The emergence of the red emissive state must then be assigned to the better passivation of the particles, thus increasing emission intensity by allowing better dispersion in solution. While electronic effects of an oxygenated surface passivation cannot be ignored, ${ }^{55,80,81}$ it is more likely that the modest increase in intensity is due to less aggregation.

Although the red emissive state is quenched by aggregation at the beginning of the experiment, the electronic transition responsible for the emission can still be assumed to have an energy gap of approx. $1.80 \mathrm{eV}$ to $1.88 \mathrm{eV}(660 \mathrm{~nm}$ to $690 \mathrm{~nm}$ ) based on previous experiments. The final emissive wavelength for all $\mathrm{H}-\mathrm{Si}$ NPs treated with BPO was $650 \mathrm{~nm}$, indicating a blue shift between $10 \mathrm{~nm}$ and $40 \mathrm{~nm}$. This very small blue shift indicates that the core must not be significantly reduced in size. Using the effective mass approximation discussed in chapter 2, a blue shift of 10 to $40 \mathrm{~nm}$ would only require a reduction in core size from 2.06 $\mathrm{nm}$ to $1.90 \mathrm{~nm}$. Since the nearest neighbor distance of crystalline $\mathrm{Si}$ is $0.235 \mathrm{~nm}$, this very small blue shift indicates that the BPO has only interacted with the surface of the crystalline core.

The FTIR analysis shows that for all treated BPO samples there is an increase in Si-O-Si stretching that occurs in conjunction with the reduction of the 
$\mathrm{Si}-\mathrm{H}$ stretching, however there is no evidence of BPO remaining on the surface after the treatment. The XPS analysis also indicates that the particles are being oxidized throughout the treatment, with the extent of oxidation increasing with increasing BPO concentration. This trend of increasing oxidation of the Si NPs with increasing BPO concentration does not, however, result in an expected blue shifting of the red emissive peak due to reduction of core size. This supports the argument that the BPO treatment only interacts with the $\mathrm{Si}-\mathrm{H}$ surface, resulting in more colloidally stable particles.

Previous Raman studies have shown that the H-Si NPs produced via our high temperature disproportionation method results in $\mathrm{Si}^{0}$ particles that have both crystalline $\mathrm{Si}^{0}$ and amorphous $\mathrm{Si}^{0}$ present. ${ }^{56}$ The crystalline $\mathrm{Si}^{0}$ domains of these particles were determined to be $2.55 \mathrm{~nm}$ from the Raman analysis but the percent crystallinity was determined to be only $55 \%$. Particle size determination via TEM of the H-Si NPs has been attempted but cannot be done due to the significant aggregation of the particles. However, in previous work similarly produced $\mathrm{H}-\mathrm{Si}$ NPs underwent a surface treatment via hydrosilylation reaction with 1-decene to produce decane terminated Si NPs has resulted in well dispersed particles that are $5 \mathrm{~nm}$ in size via TEM with no oxidation present in the FTIR spectrum. ${ }^{14}$ The total physical size of these particles is much larger than the size of the crystalline core determined spectroscopically via PL and Raman, suggesting crystalline sizes of $2.06 \mathrm{~nm}$ (Chapter 2) and $2.55 \mathrm{~nm}^{56}$ respectively. This discrepancy can be explained by the incorporation of amorphous $\mathrm{Si}^{0}$ that is being included in the TEM 
size determination. If the particles are partially amorphous, especially at the surface, the oxidation of that surface would not impact the size of the crystalline core or impart significant electronic effects on the core. This would, however, increase the stability of the particles in solution and the particles would be less aggregated. As suggested by others ${ }^{77-79}$ the significant decrease in particle aggregation alone could be the reason for the increased PL emission from the BPO treated particles.

\subsection{Conclusions}

$\mathrm{H}$-Si NPs form an unstable suspension that is prone to aggregation and significantly reduces the emission intensity of the particles, thus surface treatments that provide better colloidal stability without imparting significant electronic effects on the particles is desired. The treatment of the particles with benzoyl peroxide in under UV irradiation increase the rate of surface passivation without significantly increasing oxidation of the core. The $10 \mathrm{mM}$ treatment under UV irradiation for 24 hours resulted the most colloidally stable suspension that showed the greatest increase in $650 \mathrm{~nm}$ emission intensity without substantial blue shifting of the emissive wavelength from the starting H-Si NPs. While the particles did show surface oxidation via FTIR and XPS analysis, the emission wavelength was unaffected by this oxidation and the decreased aggregation from the BPO treated particles greatly increased the emission intensity. 
Chapter 4: Investigation into magnesiothermic reduction of silicon oxides as a synthetic route to free-standing nanocrystalline silicon

\subsection{Introduction}

Si NPs have been synthesized in many different ways. The major routes to producing Si NPs are gas phase reduction of $\mathrm{SiH}_{4},{ }^{19,45}$ solution phase oxidation reactions of silicon polyanions (e.g., $\mathrm{Si}_{4}{ }^{4-}$ ) with conventional chemical oxidizing agents, ${ }^{46-49}$ laser ablation of bulk $\mathrm{Si}^{0}$ in solution, ${ }^{50,51}$ high temperature annealing of silicon suboxide polymers ${ }^{14,52,53}$ followed by subsequent chemical etching, and solution phase reductions of silanes (e.g., $\mathrm{HSiCl}_{3}$ and others) with conventional chemical reducing agents. ${ }^{54,55}$ Physical methods of production have been shown to produce either predictably-sized particles with narrow size distributions in low synthetic yields (e.g., by laser ablation), or very wide size distributions of particles in high yields (e.g., by ball milling silicon wafers) ${ }^{82,83}$ but none has been able to combine the two. Gas phase reduction of $\mathrm{SiH}_{4}$ can produce very narrow size distributions, but only in small yields. Electrochemical etching, high temperature annealing, and solution oxidation/reduction methods generally result in larger yields that could be scalable. However these methods are in general limited in the range of achievable sizes.

We have shown in the previous chapters that the high temperature reductive annealing of silicon suboxides (e.g., $\mathrm{HSiO}_{1.5}$ ) can be used to produce nanocrystalline $\mathrm{Si}^{0}$ domains in $\mathrm{SiO}_{2}$ matrix. ${ }^{14,52,53,62}$ The oxidized matrix can then 
be etched away using hydrofluoric acid to liberate the particles into the aqueous etching solution and subsequent phase transfer into a non-polar solvent is then done to isolate hydrogen terminated Si NPs (H-Si NPs). This method has many benefits, like low-cost starting materials for the production of the suboxide polymer, however there is very little control over particle sizes using this method. The typical synthesis produces particles in the range of 4 to $5 \mathrm{~nm}$ in diameter when annealing at a temperature between $900^{\circ} \mathrm{C}$ and $1100^{\circ} \mathrm{C}$ for 1 hour or longer, but the size and crystallinity of the Si NPs cannot be greatly increased at this temperature by the time of annealing.

If we would like to increase the absorption wavelength onset of the Si NPs for applications involving light absorption (e.g., photovoltaics or photosensitization), being able to synthesize larger core sizes via another method is needed. Growth of the silicon domains via high temperature reductive annealing is limited in our lab by the $1100^{\circ} \mathrm{C}$ maximum temperature of our furnace using the standard reductive annealing method. Reaction temperatures greater than $1100^{\circ} \mathrm{C}$ can produce larger silicon particles up to $12 \mathrm{~nm}$ at $1350^{\circ} \mathrm{C}$, but above this temperature there has been shown to be a significant increase in the size polydispersity to the extent that a particle size determination cannot be made with TEM. ${ }^{53}$ To overcome some of the limitations on particle size from this method, we hypothesized that addition of a reducing metal to the $\left[\mathrm{HSiO}_{1.5}\right]_{\mathrm{n}}$ precursor polymer could lower the maximum temperature required to produce Si NPs and 
simultaneously increase the maximum particle size to a target range of 10 to 50 $\mathrm{nm}$.

\subsection{Experimental}

\subsubsection{Materials}

Trichlorosilane (Alfa Aesar), absolute ethanol (TCl), and 49wt\% hydrofluoric acid in water (Sigma-Aldrich), 325 mesh Magnesium powder (Alfa Aesar) and $\mathrm{NaCl}$ (J.T. Baker) were purchased and used as received unless otherwise specified. LUDOX HS-40 and TM-40 were purchased from Sigma-Aldrich and used as received unless specified. NanoXact $80 \mathrm{~nm} \mathrm{SiO}_{2} \mathrm{NPs}$ were purchased from nanoComposix as a dry powder and used as received. All solvents were purchased from Fisher Scientific and also used without further purification (as received) unless specified.

\subsubsection{Nanocrystalline Silicon from magnesiothermic reduction}

Nanocrystalline silicon (nc-Si) was produced from high temperature reductive annealing of $\left[\mathrm{HSiO}_{1.5}\right]_{n}$ with addition of magnesium under flowing $\mathrm{N}_{2}$ gas in an alumina crucible. The $\left[\mathrm{HSiO}_{1.5}\right]_{\mathrm{n}}$ polymer was synthesized using a previously reported method ${ }^{14,62}$ and is detailed in chapter 1 . Briefly, trichlorosilane was added to an Ar filled flask in an ice bath and water $(18 \mathrm{M} \Omega \bullet \mathrm{cm})$ was subsequently added to initiate a hydrolysis and subsequent polycondensation reaction to produce the desired $\left[\mathrm{HSiO}_{1.5}\right]_{\mathrm{n}}$ polymer with $\mathrm{HCl}$ gas as a byproduct. The product was dried 
and then stored under Ar for further use. For the magnesiothermic reduction, the starting mass of the dried $\left[\mathrm{HSiO}_{1.5}\right]_{\mathrm{n}}$ was held constant at 0.5 grams and the $\mathrm{Mg}$ added was varied such that the molar ratio of $M g$ to $O$ was 1:5 to 2:1, assuming the Si monomer stoichiometry as $\left[\mathrm{HSiO}_{1.5}\right]_{\mathrm{n}}$. The temperature for all experiments was increased at a rate of $20{ }^{\circ} \mathrm{C}$ per min until the desired maximum temperature was reached, then the temperature was held constant for the desired amount of time. The temperature was then steadily decreased over a period of $1 \mathrm{hr}$ to ambient laboratory temperature (at approximately $20^{\circ} \mathrm{C} / \mathrm{min}$ ). The annealed powder was ground again to a fine powder in a mortar and pestle and stored in a scintillation vial under Ar for further analysis and workup.

For experiments adding a heat sink, the reaction mixture of $1: 1 \mathrm{Mg}$ to $\mathrm{O}$ was mixed thoroughly mixed with an equal mass of $\mathrm{NaCl}$. In a typical experiment 0.50 $\mathrm{g}$ of $\left[\mathrm{HSiO}_{1.5}\right]_{\mathrm{n}}, 0.35 \mathrm{~g}$ of $\mathrm{Mg}$ powder, and $0.85 \mathrm{~g}$ of $\mathrm{NaCl}$ would be ground to a homogenous powder in a mortar and pestle prior to annealing. The mixture was then heated to $650^{\circ} \mathrm{C}$ at $20^{\circ} \mathrm{C} /$ minute and held at that temperature for 1 hour, followed by cooling at a rate of $20^{\circ} \mathrm{C} /$ minute back to the ambient temperature. The reaction mixture was then ground again in a mortar and pestle to a fine powder and stored under Ar for further use.

For magnesiothermic reduction of $\mathrm{SiO}_{2}$ spheres, three sources were used: commercially available LUDOX TM-40 (40wt\% $\mathrm{SiO}_{2}$ in $\mathrm{H}_{2} \mathrm{O}$ stabilized by $\left.\mathrm{NaOH}\right)$, NanoXact $\mathrm{SiO}_{2}$ spheres as a dry powder, and $\mathrm{SiO}_{2}$ spheres from a modified literature method based on the Stober synthesis. Briefly, $1.0 \mathrm{~mL}$ Millipore $\mathrm{H}_{2} \mathrm{O}, 1$ 
$\mathrm{mL} 30 \mathrm{wt} \% \mathrm{NH}_{3} \mathrm{OH}, 47 \mathrm{~mL}$ absolute $\mathrm{EtOH}$ were stirred vigorously in a $100 \mathrm{~mL}$ round bottom flask for 5 minutes. $1.0 \mathrm{~mL}$ of TEOS was then added and the mixture was then stirred vigorously overnight (18 hours total). The cloudy suspension was then titrated with $1.0 \mathrm{M} \mathrm{HCl}$ to a pH of 7 and then centrifuged at $18 \mathrm{k} \mathrm{rpm}$ for 20 minutes. The supernatant was discarded and the solids re-dispersed in a 7:3 EtOH: $\mathrm{H}_{2} \mathrm{O}$ solution and the centrifugation process was repeated. After 3 washing cycles the solid pellet was dried in a $60^{\circ} \mathrm{C}$ drying oven for 2 days and was ready for further use.

The reduction of the $\mathrm{SiO}_{2}$ spheres followed the same procedures as with the heat sink with the exception of the total masses of reactants. For these experiments, $0.30 \mathrm{~g}$ of both Stober $\mathrm{SiO}_{2}$ particles, $0.30 \mathrm{~g}$ of LUDOX TM40, and $0.10 \mathrm{~g}$ of NanoXact were used with all other ratios remaining the same. The reaction was conducted at $650^{\circ} \mathrm{C}$ for 1 hour with heating and cooling rates of $20^{\circ} \mathrm{C} / \mathrm{min}$.

\subsubsection{Washing and Etching procedure}

For the nanocrystalline silicon prepared from magnesiothermic reduction, the $\mathrm{MgO}$ and $\mathrm{Mg}_{2} \mathrm{Si}$ products, as well as any unreacted $\mathrm{Mg}$, were removed by soaking the annealed powders in $1.0 \mathrm{M} \mathrm{HCl}$ for $2 \mathrm{hr}$ under vigorous stirring. It should be noted that even if the $M g$ is kept as a limiting reagent during the metallothermic reduction reaction, there is usually some $\mathrm{Mg}_{2} \mathrm{Si}$ byproduct that develops. 
CAUTION: $\mathrm{Mg}_{2} \mathrm{Si}$ reacts with aqueous $\mathrm{HCl}$ to produce silane gas. Silane is a colorless, flammable and poisonous gas, with a strong repulsive odor. It is easily ignited in air, reacts with oxidizing agents, is very toxic by inhalation, and is a strong irritant to skin, eyes and mucous membranes. The typical total reactant masses in these experiments were less than $1 \mathrm{~g}$ and produced only small amounts (less than $0.2 \mathrm{~g}$ ) of $\mathrm{Mg}_{2} \mathrm{Si}$. Under these conditions, it was appropriate to do the product etching in a fume hood with no other flammables present. At higher Mg:O ratios in the reactant mixture there will be an increase in the $\mathrm{Mg}_{2} \mathrm{Si}$, and thus more precaution should be used. If attempting to scale this process up, please be advised that an explosion barrier should be set up between you and the etching solution.

At the end of the $2 \mathrm{hr}$ aqueous $\mathrm{HCl}$ etch, the suspended product was then centrifuged at $4400 \mathrm{rpm}$ for $20 \mathrm{~min}$ in a polypropylene centrifuge tube and the resulting pellet was dried in air. The washed powder was then etched in a 1:1:1 mixture of $\mathrm{EtOH}: \mathrm{H}_{2} \mathrm{O}: \mathrm{HF}$ using $5 \mathrm{~mL}$ of each; the washed powder was stirred in the HF etching solution was stirred for $1 \mathrm{hr}$. Afterwards, the hydrogen terminated nanocrystalline silicon was extracted into $40 \mathrm{~mL}$ of non-polar solvent (either hexanes or toluene) by phase transfer. Since the resulting suspension is wet and contains some ethanol, the suspension containing the H-Si NPs was then centrifuged in a glass centrifuge tube, supernatant discarded, then re-dispersed into dry toluene then purged with $\mathrm{Ar}$ for 30 minutes and stored under $\mathrm{Ar}$ for future use. 


\subsubsection{Characterization of nc-Si}

XRD spectroscopy was conducted on a Rikagu Ultima IV X-ray diffractometer using an x-ray wavelength of $1.54 \AA$ in Bragg-Brentano geometry (powder diffraction, focused beam). The PDXL2 software package (version 2.7) was used for peak identification using the Crystallography Open Database (COD). The raw data was modeled using PDXL2 software to give calculated values for each peak ( $2 \theta$ peak position, integrated intensity, peak width, etc.). Crystallite sizes $(\tau)$ were calculated using the Scherrer equation (equation 4.1),

$$
\tau=\frac{K \lambda}{\beta \cos \theta}
$$

where $\mathrm{K}$ is a shape factor ( 0.90 is commonly used for mostly spherical particles), $\lambda$ is the $\mathrm{x}$-ray wavelength in $\mathrm{nm}\left(0.1541 \mathrm{~nm}\right.$ for $\mathrm{Cu} \mathrm{K}_{\alpha}$ radiation used $), \beta$ is the peak width at half maximum height for each reflection (units of $B$ ?), and $\theta$ is the Bragg

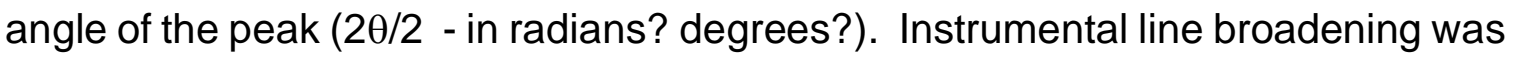
accounted for by measuring the FWHM for the (111), (220), and (311) reflections of a large grained polycrystalline silicon standard under identical instrumental conditions.

\subsubsection{TEM}

A Tecnai-F20 transmission electron microscope (FEI, Hillsboro, OR) operating at $200 \mathrm{kV}$ was used to image the nanocrystalline silicon reaction products. TEM samples were prepared by drop-casting a suspension of NPs onto 
a carbon coated Cu grid and then allowed to dry in a vacuum chamber to ensure removal of solvent. Particle sizes reported were determined from a minimum of 200 particles from multiple micrographs using ImageJ software.

\subsubsection{SEM}

A Zeiss Sigma VP FEG SEM was used under accelerating voltages of 2.55 $\mathrm{kV}, 5 \mathrm{kV}$, and $10 \mathrm{kV}$. A $3 \mu \mathrm{m}$ aperture was used without high current. Frame averaging was varied from $\mathrm{N}=4$ to $\mathrm{N}=64$. The detector used was a secondary electron detector (Everhartt-Thornley). Particle sizes reported were determined from a minimum of 200 particles from multiple micrographs using ImageJ software.

\subsection{Results and Discussion}

\subsubsection{Preliminary reduction of $\left[\mathrm{HSiO}_{1.5}\right]_{n}$ with magnesium metal}

The initial goal of this project was to increase the average particle size of the Si NPs obtained in a typical solid state reductive annealing synthesis from 5 $\mathrm{nm}$, up to 10 to $50 \mathrm{~nm}$ in size. We hypothesized that the addition of $\mathrm{Mg}$ metal to the $\left[\mathrm{HSiO}_{1.5}\right]_{\mathrm{n}}$ precursor would increase the particle size by increasing the amount of $\mathrm{Si}$ atoms that are reduced compared to the high temperature reductive annealing of the precursor alone. To do this we adapted a previous literature method that was used to reduce diatoms (macroscopically organized $\mathrm{SiO}_{2}$-based organisms) to porous silicon replica structures. ${ }^{84}$ 

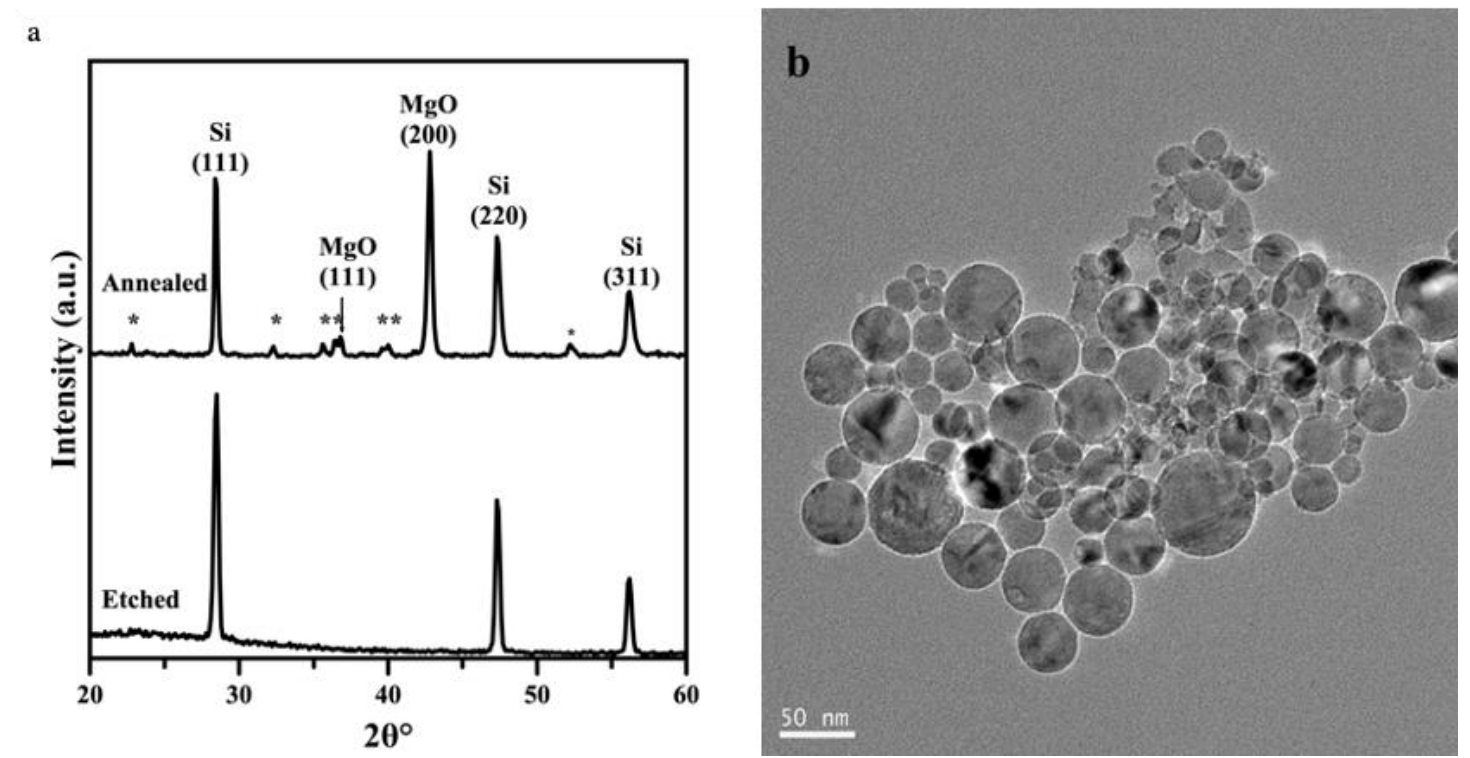

Figure 4.1: XRD patterns and TEM images from $\mathrm{Mg}$ reduction of $\left[\mathrm{HSiO}_{1.5}\right]_{\mathrm{n}}$. (a) XRD patterns for the annealed product directly after the reaction (upper trace) and post etching procedures (bottom trace) for the initial synthesis of $\mathrm{H}$-Si NPs from $\mathrm{Mg}$ reduction of [HSiO ${ }_{1.5}$ ]n precursor. (b) Representative TEM image of 33 $\mathrm{nm} \mathrm{H}$-Si NPs observed after the post etching procedures.

In the first magnesiothermic reduction reaction that we attempted, nanocrystalline silicon was produced from the reaction of the $\left[\mathrm{HSiO}_{1.5}\right]_{\mathrm{n}}$ precursor and $\mathrm{Mg}$ powder at a $\mathrm{Mg}: \mathrm{HSiO}_{1.5}$ ratio of $1.5: 1$ at $650^{\circ} \mathrm{C}$ (the melting point of $\mathrm{Mg}$ ) for 2.5 hours. The powder XRD pattern for the resulting powder, prior to etching, has a mixture of products, as can be seen in Figure 4.1a. The XRD pattern clearly shows reflections at $28.5,47.5$, and $5620^{\circ}$ associated with the (111), (220), and (311) lattice planes of crystalline $\mathrm{Si}$. The pattern also shows reflections at 36 and $422 \theta^{\circ}$ that are associated with the (111) and (220) lattice planes of MgO. There are no observed reflections for $\mathrm{Mg}$, indicating the complete oxidation of the $\mathrm{Mg}$ starting material. The partially oxidized $\left[\mathrm{HSiO}_{1.5}\right]_{\mathrm{n}}$ starting material is not 
crystalline, which would result in a broad peak centered between 20 and $252 \theta^{\circ}$ if it were present (due to the short range order of the amorphous material). There is no evidence in the annealed powder XRD pattern that there is any amorphous material, which indicates that all of the hydridosilicate-polymer starting material has been converted to products. The minor peaks denoted with * in the XRD pattern following annealing are reflections indicating the presence of $\mathrm{Mg}_{2} \mathrm{SiO}_{4}$, an unexpected byproduct of the reaction. A 2 hour etch with $1 \mathrm{M} \mathrm{HCl}$ was afterwards done to remove the $\mathrm{MgO}$, and was followed by etching with an aqueous $\mathrm{HF}$ solution to remove the $\mathrm{Mg}_{2} \mathrm{SiO}_{4}$, leaving only crystalline silicon. The XRD pattern following etching in Figure 4.1a shows only the remaining Si reflections; however there is a broad peak centered around $232 \theta^{\circ}$ that indicates a small amount of amorphous material has developed, possibly due to a small oxide layer on the $\mathrm{Si}$ surface after exposure to air. Figure $4.1 \mathrm{~b}$ shows a representative TEM image of the Si particles that were isolated after the etching procedure. These particles are crystalline, spherical, and have a very large range of sizes from approx. $10 \mathrm{~nm}$ to $60 \mathrm{~nm}$ in diameter. The measured (200 particles) average particle diameter was determined to be $33 \mathrm{~nm} \pm 13 \mathrm{~nm}$ from TEM images. Scherrer analysis of the line broadening in the XRD pattern (described above in methods) determined that the average Si crystallite size for the etched product was $34 \mathrm{~nm}$, in good agreement with the size from TEM analysis. To date, almost all reports of silicon products from magnesiothermic reduction of $\mathrm{SiO}_{2}$ have produced large porous silicon structures, ${ }^{84-91}$ however this promising initial result shows that smaller, 
freestanding Si NPs can be achieved via this process. The observed Si NPs do not appear to be porous in the TEM images from this sample, consistent with the measured XRD grain size.

\subsubsection{Effects of time, temperature, and reactant concentrations on product morphology}

The initial reaction utilized reaction conditions at the melting temperature of $\mathrm{Mg}\left(650^{\circ} \mathrm{C}\right)$, stoichiometric equivalent amounts of $\mathrm{Mg}$ to $\mathrm{O}$ (in the $\left[\mathrm{HSiO}_{1.5}\right]_{\mathrm{n}}$ precursor), and a reaction time of 6 hours. The resulting Si NPs were within the target size range; however it was hypothesized that both the size and size polydispersity could be controlled via manipulation of reaction conditions to produce particles of a desired size with more narrow size distributions. The three reaction conditions that were varied were time, temperature, and the molar ratio of reactants. It was expected that increasing any of the three variables (time, temperature, or molar ratio) should all show a trend of increasing the particle size based on previous studies. ${ }^{92}$

The time of reaction was first varied from 0 minutes to 6 hours while holding temperature and molar ratio constant. The 0 minute reaction time was observed by increasing the furnace temperature at a rate of $20^{\circ} \mathrm{C} / \mathrm{min}$ to the $650^{\circ} \mathrm{C}$ reaction temperature, and then immediately reducing the temperature at $20^{\circ} \mathrm{C} / \mathrm{min}$ to ambient temperature. For other reaction durations tested (up to 6 hours), varying the time of reaction did not result in any observable changes in crystallite size by 
powder XRD line broadening. All were observed to be approx. $30 \mathrm{~nm}$ to $35 \mathrm{~nm}$, including the 0 minute reaction time. This indicated that the reaction produced elemental silicon domains very rapidly upon reaching the melting point of $\mathrm{Mg}$, and that afterwards no further particle growth was observed.

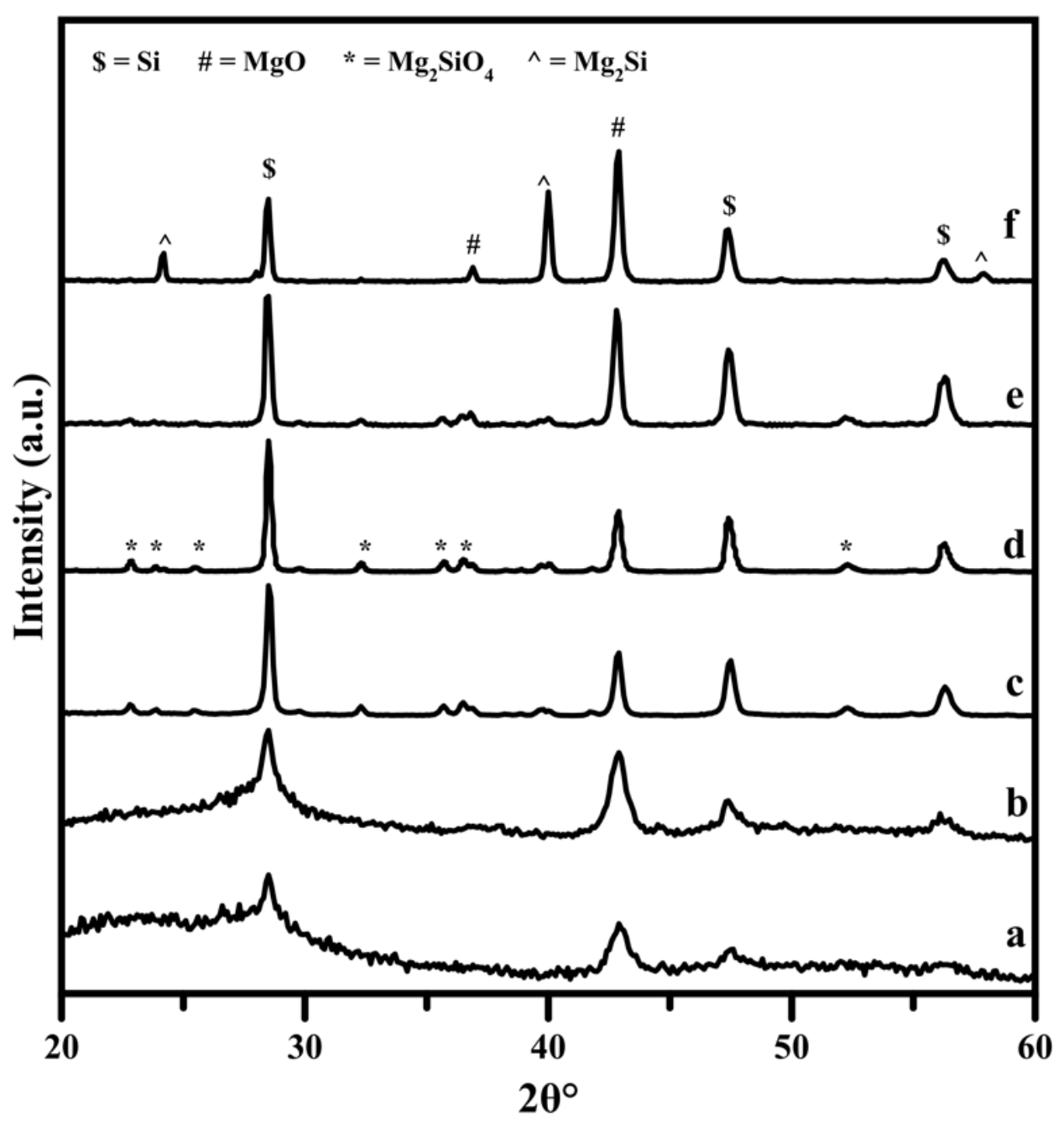

Figure 4.2: XRD patterns of the annealed product mixture with varying reactant ratios. The reactant ratios of $\mathrm{Mg}$ to $\mathrm{O}$ increase from bottom to top as follows: (a) 1:5, (b) 2:5, (c) 3:5, (d) 4:5, (e) 1:1, and (f) 2:1. The observed peaks are denoted with symbols corresponding to crystalline material indicated at the top. 
Next, reactant concentrations were varied to try and control particle size, while holding the time constant at 0 hours, and the temperature at $650^{\circ} \mathrm{C}$. The molar ratio of $\mathrm{Mg}: \mathrm{O}$ was varied from 1:5 ( $\mathrm{Si}$ in excess) to 2:1 (Mg in excess) and the XRD patterns from the resulting powders can be seen in Figure 4.2 above. For molar ratios of $\mathrm{Mg}: \mathrm{O}$ of $1: 5$ and 2:5 (Figure 4.2a and 4.2b respectively) the XRD patterns show the expected products $\mathrm{MgO}$ and $\mathrm{Si}$, but also have a significant portion of amorphous material that is presumably from the $\left[\mathrm{HSiO}_{1.5}\right]_{\mathrm{n}}$ polymer that is unreacted. The Si crystallite size from XRD line broadening for the 1:5 molar ratio was $8 \mathrm{~nm}$ and for the 2:5 molar ratio was $13 \mathrm{~nm}$. When the ratio of $\mathrm{Mg}$ was increased to $3: 5$ (Figure 4.2c) the pattern shows peaks from $\mathrm{MgO}, \mathrm{Si}$, and $\mathrm{Mg}_{2} \mathrm{SiO}_{4}$; further, little to no amorphous material was detected. The line broadening gives a Si crystallite size of $26 \mathrm{~nm}$ for the $3: 5$ molar ratio. As the ratio of Mg was increased further to 4:5 and 1:1 (Figure $4.2 \mathrm{~d}$ and e), the XRD patterns remained qualitatively the same as for the 3:5 ratio. The only products detected were the $\mathrm{MgO}, \mathrm{Si}$, and $\mathrm{Mg}_{2} \mathrm{SiO}_{4}$ with no amorphous phase, and the Si crystallite sizes were determined from XRD line broadening to be $34 \mathrm{~nm}$ and $32 \mathrm{~nm}$ for the 4:5 and 1:1 ratios respectively. When the molar ratio of $\mathrm{Mg}: \mathrm{O}$ was still further increased to 2:1 (Figure 4.2f) there are products of $\mathrm{MgO}, \mathrm{Si}$ and $\mathrm{Mg}_{2} \mathrm{Si}$ with no apparent $\mathrm{Mg}_{2} \mathrm{SiO}_{4}$ or unreacted $\mathrm{Mg}$ observed in the pattern; the Si crystallite size by line broadening for Si was $31 \mathrm{~nm}$.

Overall, these experiments showed that there is an observed increase in domain size with increasing $\mathrm{Mg}: \mathrm{O}$ ratio when the hydridosilicate starting material 
is in excess. Further, when $\mathrm{Mg}$ is significantly limiting, a large amount of amorphous material remains in the reaction products, presumably from unreacted [HSiO $\left.{ }_{1.5}\right]_{n}$ starting material. However, when $\mathrm{Mg}$ is excess, particle size no longer changes with increased $\mathrm{Mg}$ content, only the presence of Mg-oxide byproducts is increased - this indicates that the reaction stoichiometrically converts the partially oxidized $\mathrm{Si}$ atoms in the starting material to fully reduced $\mathrm{Si}$ atoms in the reaction products.

Finally, the temperature of the reaction was also varied and XRD line broadening was analyzed. The heating and cooling rate was held constant at $20^{\circ} \mathrm{C} / \mathrm{min}$ and maximum temperatures of $500^{\circ} \mathrm{C}, 550^{\circ} \mathrm{C}, 600^{\circ} \mathrm{C}$, and $650^{\circ} \mathrm{C}$ were held for 1 hour before cooling. The molar ratio of $\mathrm{Mg}: \mathrm{O}$ was also held constant at 1:1. XRD patterns were observed for each reaction mixture post annealing without further washing procedures and can be seen in Figure 4.3. When the maximum reaction temperature was $500^{\circ} \mathrm{C}$ the XRD pattern (Figure 4.3a) shows reflections for unreacted $\mathrm{Mg}$ at $32.2,34.4,36.6,47.8$, and $57.42 \theta^{\circ}$ representing the (100), (002), (101), (102), and (110) lattice planes respectively. There is also a broad feature centered at approx. $232 \theta^{\circ}$ indicating there is amorphous material presumably from the $\left[\mathrm{HSiO}_{1.5}\right]_{\mathrm{n}}$ starting material. Reflections at 24.2 and $40.12 \theta^{\circ}$ associated with the (111) and (220) planes for $\mathrm{Mg}_{2} \mathrm{Si}$ and a broad peak at $42.82 \theta^{\circ}$ 


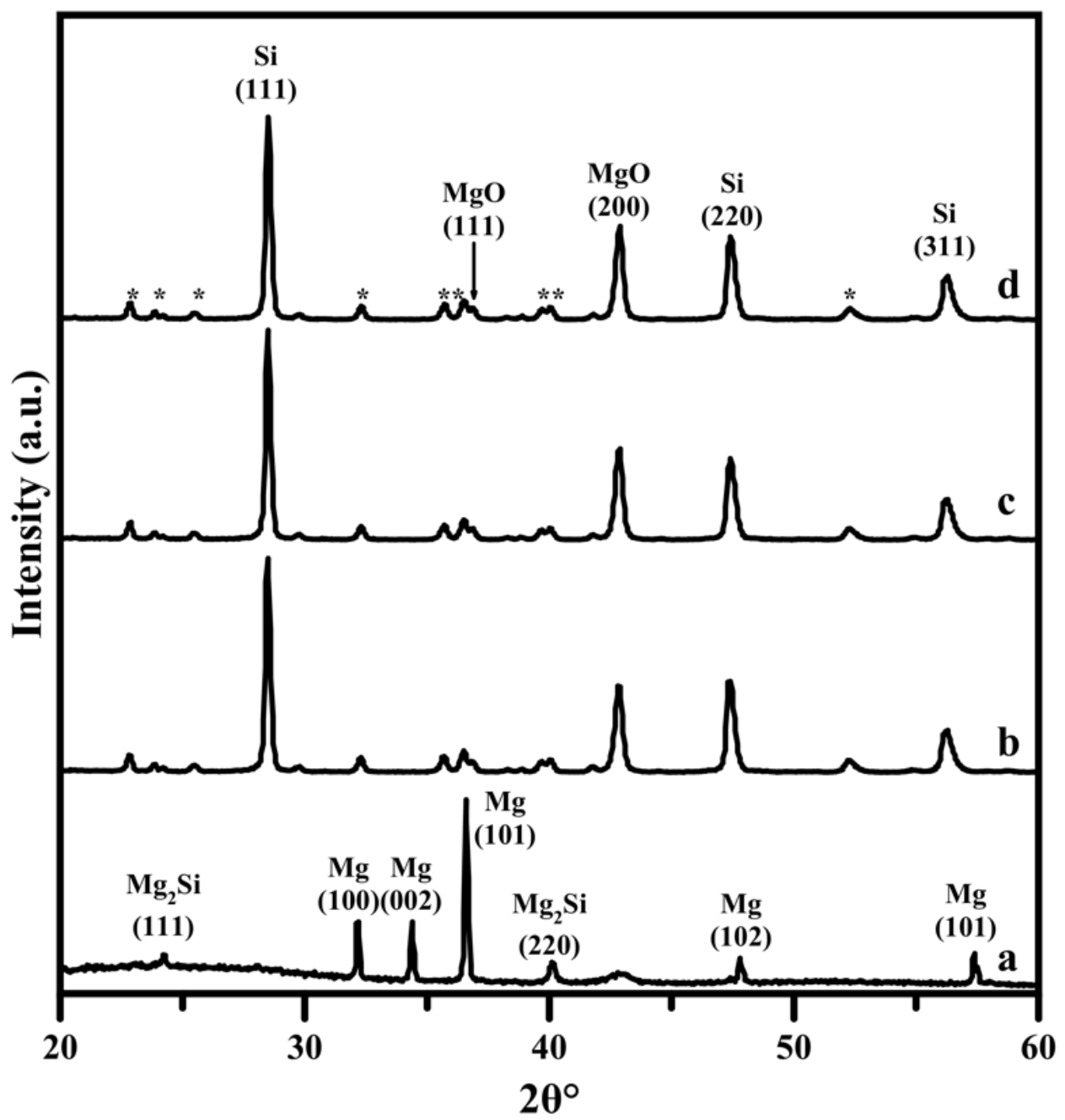

Figure 4.3: XRD patterns of the annealed product mixture with varying reaction temperature. The reaction temperatures are (a) $500^{\circ} \mathrm{C}$, (b) $550^{\circ} \mathrm{C}$, (d) $600^{\circ} \mathrm{C}$, and (d) $650^{\circ} \mathrm{C}$. The reflections indicated by ${ }^{*}$ are associated with $\mathrm{Mg}_{2} \mathrm{SiO}_{4}$.

associated with the (200) lattice plane of $\mathrm{MgO}$ was observed. No crystalline $\mathrm{Si}$ peaks were observed from the $500^{\circ} \mathrm{C}$ reaction temperature. At maximum reaction temperatures of $550^{\circ} \mathrm{C}, 600^{\circ} \mathrm{C}$, and $650^{\circ} \mathrm{C}$ (Figures $4.3 \mathrm{~b}, 4.3 \mathrm{c}$, and $4.3 \mathrm{~d}$ ) the XRD patterns are very similar. There are the observed reflections for $\mathrm{Si}, \mathrm{MgO}$ and 
$\mathrm{Mg}_{2} \mathrm{SiO}_{4}$ products as indicated in Figure 4.3. The line broadening analysis using the Scherer equation gave Si crystallite sizes of $32 \mathrm{~nm}, 31 \mathrm{~nm}$ and $34 \mathrm{~nm}$ for the reactions at $550^{\circ} \mathrm{C}, 600^{\circ} \mathrm{C}$, and $650^{\circ} \mathrm{C}$ respectively.

The results from these experiments, where only the temperature varied, indicate that there is a minimum threshold temperature that must be reached in order to accomplish conversion of the hydridosilicate precursor to elemental silicon; this temperature is between $500^{\circ} \mathrm{C}$ and $550^{\circ} \mathrm{C}$. Above $550^{\circ} \mathrm{C}$, however, there is no observed trend in the resultant particle sizes, contrary to our hypothesis. It should also be noted that additional experiments conducted at $550^{\circ} \mathrm{C}$ with reaction times of times of 0 and 6 hours showed no discernible difference in the size or composition of the annealed products in comparison to $1 \mathrm{hr}$ annealing; this (again) indicates that this reaction is occurring rapidly, even when the reaction temperature is well below the melting point of $\mathrm{Mg}\left(650^{\circ} \mathrm{C}\right)$.

Overall, the magnesiothermic reduction reactions conducted at or above $550^{\circ} \mathrm{C}$ all produced nano-crystalline Si between 10 and $34 \mathrm{~nm}$, according to the domain sizes extracted from XRD line broadening. However, after etching the reaction products to isolate the crystalline domains, the TEM images of the isolated nano-crystalline $\mathrm{Si}$ (Figure 4.4a) did not show the same morphology as was observed in our initial synthesis (Figure 4.1b). There were few if any freestanding spherical particles and the majority of the mass was composed of large, irregularly shaped material. It was surmised that this could be due to particle aggregation and then fusing that occurred during the reduction process. An alternate possibility 
would be aggregation after etching out of the solid, as observed for the smaller $\mathrm{H}$ Si NPs in the earlier chapters, however we tried post-passivation by hydrosilylation to see if improved ability to isolate free-standing un-aggregated H-Si NPs but the same structures were observed.

\subsubsection{Addition of heat sink to reduce crystallite fusion}

Domain aggregation of nano-silicon within the initial (or as-synthesized) solid has been observed in numerous studies using larger $\mathrm{SiO}_{2}$ structures as templates for magnesiothermic reduction to $\mathrm{Si}^{84-91}$ The reduction reaction itself is exothermic (equation 4.2) and will release a large amount of heat once the reaction is initiated. This is most likely the reason that the 0 minute time point still resulted in a complete conversion to crystalline products even at temperatures well below the melting point of $\mathrm{Mg}$. Previous reports have shown that $\mathrm{Mg}$ vapor is the reactive species in this reaction ${ }^{84,86,92-94}$ and at $550^{\circ} \mathrm{C}$ there must be enough vapor pressure to initiate the reaction. The extra heat generated from the reduction reaction could then create a micro-environment that has a local temperature much higher than that of the overall reaction vessel. This could increase the vapor pressure of $\mathrm{Mg}$ in the local reaction environment while also providing enough excess energy for individual $\mathrm{Si}$ crystallites to fuse together after formation and become multi-grained large structures. 


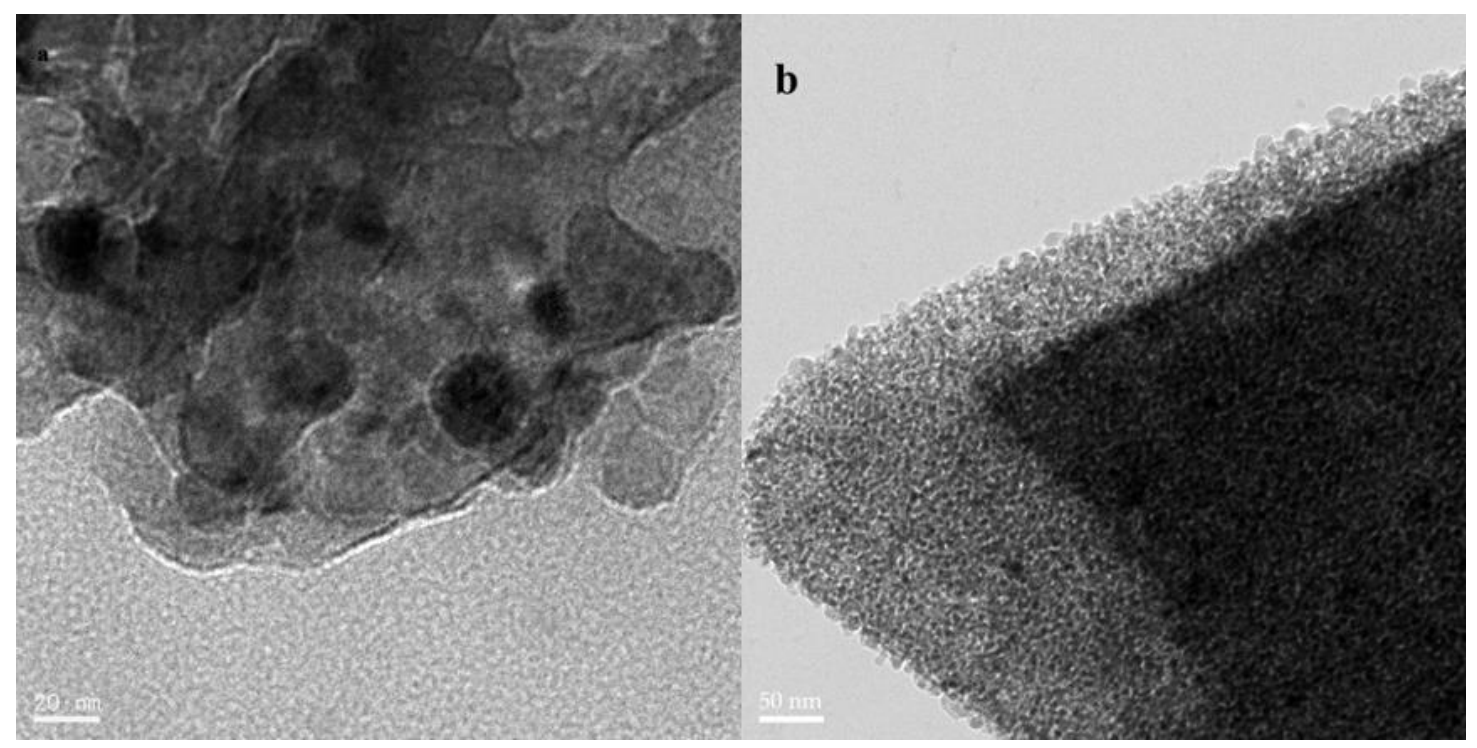

Figure 4.4: TEM images of $\mathrm{H}$-terminated $\mathrm{nc}-\mathrm{Si}$ annealed with and without $\mathrm{NaCl}$. (a) A representative TEM image from the temperature change and molar ratio change reductions without $\mathrm{NaCl}$ as a heat sink (XRD pattern shown above in Figure 4.3b). (b) Representative TEM image from reduction reactions conducted with the addition of $\mathrm{NaCl}$ as a heat sink.

We hypothesized that we could avoid fusion of the Si nanocrystals by better distributing the heat generated in the exothermic reaction with addition of $\mathrm{NaCl}$ (50 $w t \%)$ to act as a non-reactive heat sink in the reaction mixture. A representative TEM image of the nanocrystalline silicon obtained after post-synthetic workup can be seen in Figure 4.4b. The darker portion of this image appears to be a large mass as observed before, however there are many smaller masses that appear to have deposited on the surface of the large mass, most likely during drying of the sample on the TEM grid. No fields of freestanding particles could be found so we did not calculate a size distribution from TEM; however, line broadening analysis 
of the XRD pattern (not shown) gave a Si crystallite size of $8 \mathrm{~nm}$. As an additional observation, the irregularity of the smaller masses in Figure 4.3b suggests that the initial particle size of the $\left[\mathrm{HSiO}_{1.5}\right]_{\mathrm{n}}$ material may be preserved after the reduction process.

\subsubsection{Magnesiothermic reduction of $\mathrm{SiO}_{2}$ spheres}

From the experiment with added of $\mathrm{NaCl}$ as a heat sink, we hypothesized that the morphology of the nano-crystalline silicon product may be similar to that of the starting $\left[\mathrm{HSiO}_{1.5}\right]_{\mathrm{n}}$ material. This led us to try a new strategy that would take advantage of highly monodisperse, highly oxidized silica NPs ( $\left.\mathrm{SiO}_{2} \mathrm{NPs}\right)$ as templates; $\mathrm{SiO}_{2} \mathrm{NPs}$ of a variety of sizes are commercially available, or can readily be synthesized in the lab using the Stober method described above. We expected that under appropriate synthetic conditions, magnesiothermic reaction could generate slightly smaller reduced Si NPs with retention of the spherical 


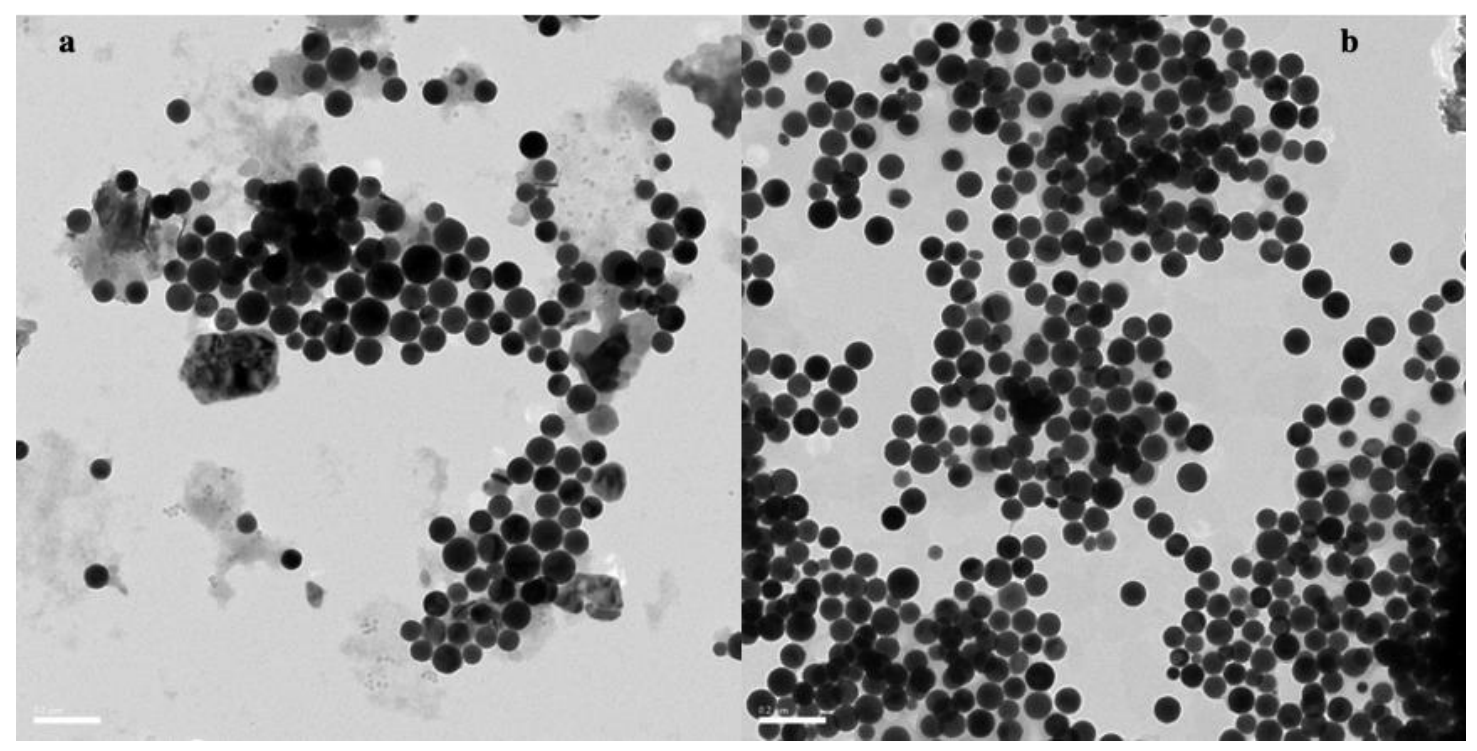

Figure 4.5: TEM images of $\mathrm{SiO}_{2}$ and $\mathrm{Si}$ NPs before and after reduction. (a) $\mathrm{SiO}_{2} \mathrm{NPs}$ with average size of $72+/-15 \mathrm{~nm}$ before reduction and (b) Si NPs with average diameter of $68+/-10 \mathrm{~nm}$ after reduction. Scale bars in both images are $200 \mathrm{~nm}$.

morphology. We further hypothesized that high local heats of reaction would result in crystalline over porous Si nanostructures. To test this we started with spherical $\mathrm{SiO}_{2}$ particles of approx. $72 \mathrm{~nm}+/-15 \mathrm{~nm}$, purchased in aqueous colloidal form from a commercial source; a TEM image of this starting material is seen in Figure 4.5a. The $\mathrm{SiO}_{2}$ particles were then reacted with $\mathrm{Mg}$ at a ratio of $1: 1 \mathrm{Mg}: \mathrm{O}$ at $650^{\circ} \mathrm{C}$ for 1 hour. A representative TEM image of the resulting Si particles after washing and etching procedures is seen in Figure 4.5b. Notably, the spherical morphology is well maintained following reduction; the average diameter for the Si particles is $68 \mathrm{~nm}+/-10 \mathrm{~nm}$, which is slightly smaller with a smaller variance as compared to the initial $\mathrm{SiO}_{2}$ particle size distribution. The XRD pattern verified the product after reduction and workup as nanocrystalline $\mathrm{Si}$, and $\mathrm{XRD}$ line broadening gave an 
average crystallite size of $63 \mathrm{~nm}$. The agreement from XRD line broadening and TEM suggests that the Si NPs after reduction are single grained particles of about $68 \mathrm{~nm}$. Since the XRD line broadening shows the average coherently scattering domain size below $100 \mathrm{~nm},{ }^{95}$ the results from this experiment would suggest that the particles are not porous and mostly free from structural defects. While there is another literature reports of the magnesiothermic reduction of $\mathrm{SiO}_{2} \mathrm{NPs}$ producing solid Si replica particles, ${ }^{96}$ obtaining solid crystalline Si structures contradicts the majority of literature that suggests this is process results in porous silicon structures. ${ }^{84-91}$

To address the inconsistency with the literature, we conducted further experiments to try and determine the porosity of the Si NPs. It was hypothesized that as the size of the initial $\mathrm{SiO}_{2}$ particle increases, there would be more porosity observed. We chose to use SEM to try and visualize the surface of the particles and to see if we could identify the presence of pores on the surface. We started with three different sizes of $\mathrm{SiO}_{2}$ : commercially available LUDOX TM-40 particles that are $22 \mathrm{~nm}$ in size, commercially available NanoXact that are $80 \mathrm{~nm}$ in size, and $\mathrm{SiO}_{2} \mathrm{NPs}$ that were made in our lab.

The SEM images from before and after reduction are presented in Figure 4.6 (following page). The LUDOX particles (Figure 4.6a) before reduction show uniformly sized particles of $22 \mathrm{~nm}+/-2 \mathrm{~nm}$ as the bottle suggests. The NanoXact particles are $75 \mathrm{~nm}+/-6 \mathrm{~nm}$ particles shown in Figure 4.6b. The $\mathrm{SiO}_{2}$ particles produced by our lab (Figure 4.6c) had an average size of $240 \mathrm{~nm}+/-26 \mathrm{~nm}$. The 


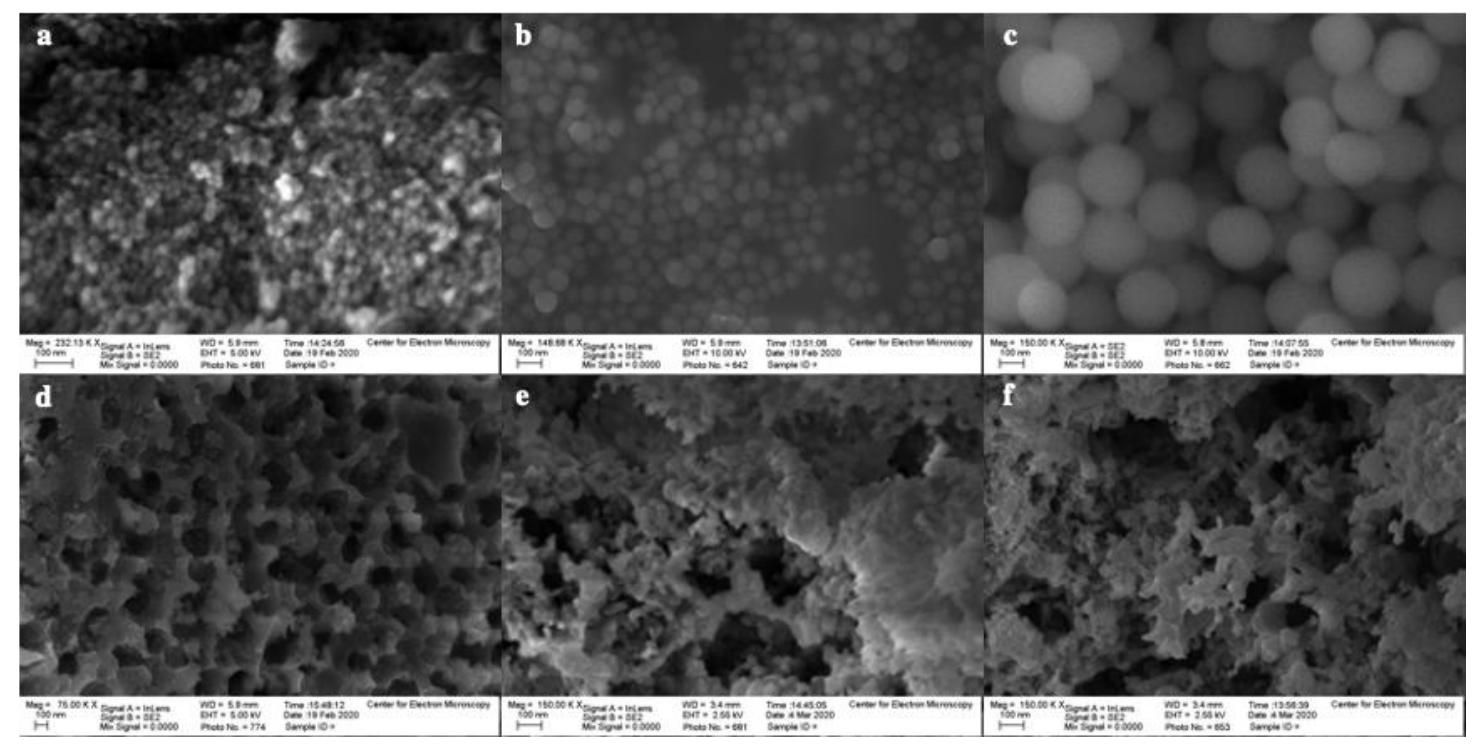

Figure 4.6: $\mathrm{SEM}$ images of $\mathrm{SiO}_{2}$ before and $\mathrm{nc}-\mathrm{Si}$ after reduction. Initial $\mathrm{SiO}_{2}$ NP from LUDOX TM-40 (a), NanoXact (b), and our own lab made particles (c). After reduction the SEM images show reduced material from LUDOX (d), NanoXact (e), and our own lab made $\mathrm{SiO}_{2}$ particles (f). Scale bars in all images are $100 \mathrm{~nm}$.

images taken after reduction and post synthetic workup (Figure $4.6 \mathrm{~d}$, e, and f) are very different structures than the starting material and much more similar to previous results from magnesiothermic reduction of the $\mathrm{HSiO}_{1.5}$ precursor detailed above. The resulting material does not contain free standing particles as was seen in Figure 4.5 for our initial synthesis using LUDOX particles, but instead is an agglomerated mass of Si domains. The XRD patterns for the reduced Si material after HF etching (corresponding to images shown in figure $4.6 \mathrm{~d}$, e, and f) show the three characteristic peaks for diamond lattice $\mathrm{Si}$ at $28.5,47.5$, and $562 \theta^{\circ}$ for all three samples. The line broadening indicates crystalline domain sizes of 16.3 
$\mathrm{nm}, 21.4 \mathrm{~nm}$, and $22.5 \mathrm{~nm}$ for the LUDOX, NanoXact, and Stober reduced samples respectively from XRD line broadening calculations.

The small domain sizes of from XRD and the large masses from the SEM images suggest the domains are fusing together upon reduction which form large

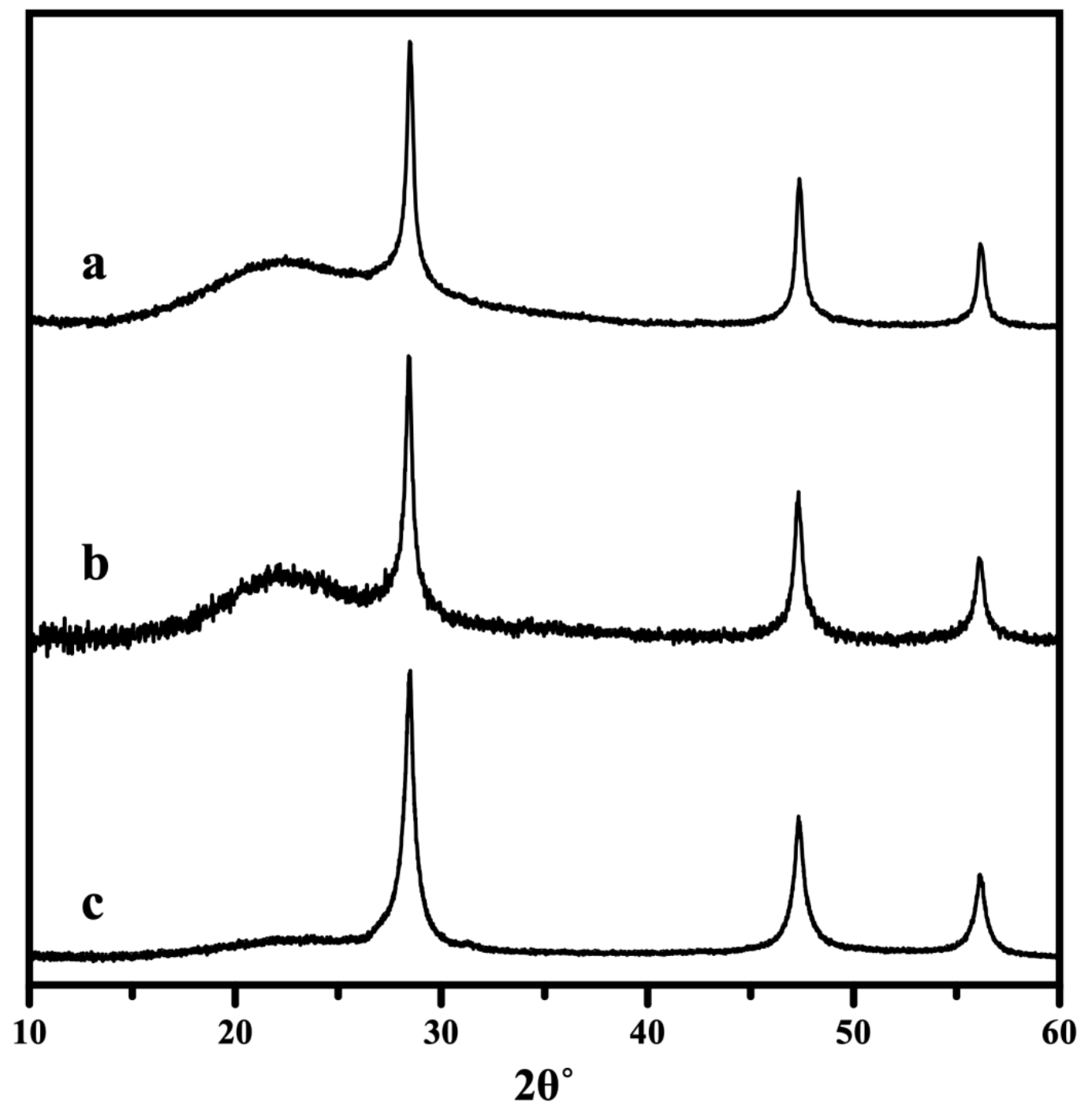

Figure 4.7: XRD patterns after the reduction of three $\mathrm{SiO}_{2}$ precursors. (a) Stober $\mathrm{SiO}_{2}$ particles, (b) NanoXact, and (c) LUDOX TM40 with magnesium and post-synthetic work up to remove byproducts. 
crystalline Si masses. The large difference between the crystalline domain size of the $\mathrm{Si}$ product and the precursor $\mathrm{SiO}_{2}$ size is more consistent with previous literature reports ${ }^{84-91}$ than the initial observations we saw in Figure 4.5 or was observed by Dasog and coworkers. ${ }^{96}$

\subsection{Conclusions}

The initial goals of the project were to increase the size and crystallinity of Si NPs from the previous $5 \mathrm{~nm}$ size limitation from the standard high temperature reductive annealing method we have employed in our lab previously. To do this we attempted to develop a method based on metallothermic reduction of silicon oxides that have lower threshold temperatures than the reductive annealing method. We were successful in demonstrating that the $\left[\mathrm{HSiO}_{1.5}\right]_{\mathrm{n}}$ precursor material can be reduced by $\mathrm{Mg}$ metal to highly crystalline $\mathrm{Si}^{0}$ at temperatures as low as $550^{\circ} \mathrm{C}$. Although the Si produced in this process is more crystalline than the $\mathrm{Si}^{0}$ produced from the high temperature reductive annealing method, it is difficult to predictably control the resultant morphology to the degree necessary for

industrial applications. We were able to show that the size and morphology of the $\left[\mathrm{HSiO}_{1.5}\right]_{\mathrm{n}}$ material could be preserved with the addition of a heat sink to absorb excess heat produced from the reaction. Unfortunately the reduction of spherical $\mathrm{SiO}_{2} \mathrm{NPS}$ had irreproducible results. In some cases the particles were freestanding $\mathrm{H}$-Si NPs with similar size and shape to the precursor $\mathrm{SiO}_{2}$ particles, 
but in most cases the $\mathrm{Si}^{0}$ crystallites formed large multi-domain crystalline silicon structures. 
Terminal References

(1) Canham, L. T. Silicon Quantum Wire Array Fabrication by Electrochemical and Chemical Dissolution of Wafers. Appl. Phys. Lett. 1990, 57, 10461048.

(2) Gonzalez, C. M.; Veinot, J. G. C. Silicon Nanocrystals for the Development of Sensing Platforms. J. Mater. Chem. C 2016, 4, 4836-4846.

(3) Xu, X.; Ma, S.; Xiao, X.; Hu, Y.; Zhao, D. The Preparation of High-Quality Water-Soluble Silicon Quantum Dots and Their Application in the Detection of Formaldehyde. RSC Adv. 2016, 6, 98899-98907.

(4) Han, Y.; Chen, Y.; Feng, J.; Liu, J.; Ma, S.; Chen, X. One-Pot Synthesis of Fluorescent Silicon Nanoparticles for Sensitive and Selective Determination of 2,4,6-Trinitrophenol in Aqueous Solution. Anal. Chem. 2017, 89, 3001-3008.

(5) Wang, H.; He, Y. Recent Advances in Silicon Nanomaterial-Based Fluorescent Sensors. Sensors (Switzerland) 2017, 17.

(6) Li, Z. F.; Ruckenstein, E. Water-Soluble Poly(Acrylic Acid) Grafted Luminescent Silicon Nanoparticles and Their Use as Fluorescent Biological Staining Labels. Nano Lett. 2004, 4, 1463-1467.

(7) Ji, X.; Peng, F.; Zhong, Y.; Su, Y.; Jiang, X.; Song, C.; Yang, L.; Chu, B.; Lee, S. T.; He, Y. Highly Fluorescent, Photostable, and Ultrasmall Silicon Drug Nanocarriers for Long-Term Tumor Cell Tracking and in-Vivo Cancer Therapy. Adv. Mater. 2015, 27, 1029-1034.

(8) McVey, B. F. P.; Tilley, R. D. Solution Synthesis, Optical Properties, and Bioimaging Applications of Silicon Nanocrystals. Acc. Chem. Res. 2014, 47, 3045-3051.

(9) Lalic, N.; Linnros, J. Light Emitting Diode Structure Based on Si Nanocrystals Formed by Implantation into Thermal Oxide. J. Lumin. 1998, 80, 263-267.

(10) Puzzo, D. P.; Henderson, E. J.; Helander, M. G.; Wang, Z.; Ozin, G. A.; Lu, Z. Visible Colloidal Nanocrystal Silicon Light-Emitting Diode. Nano Lett. 2011, 11, 1585-1590.

(11) Mastronardi, M. L.; Henderson, E. J.; Puzzo, D. P.; Chang, Y.; Wang, Z. Bin; Helander, M. G.; Jeong, J.; Kherani, N. P.; Lu, Z.; Ozin, G. A. Silicon Nanocrystal OLEDs: Effect of Organic Capping Group on Performance. Small 2012, 8, 3647-3654.

(12) Hessel, C. M.; Reid, D.; Panthani, M. G.; Rasch, M. R.; Goodfellow, B. W.; Wei, J.; Fujii, H.; Akhavan, V.; Korgel, B. a. Synthesis of Ligand-Stabilized Silicon Nanocrystals with Size-Dependent Photoluminescence Spanning Visible to Near-Infrared Wavelengths. Chem. Mater. 2012, 24, 393-401. Clark, R. J.; Aghajamali, M.; Gonzalez, C. M.; Hadidi, L.; Islam, M. A.; Javadi, M.; Mobarok, M. H.; Purkait, T. K.; Robidillo, C. J. T.; Sinelnikov, 
R.; et al. From Hydrogen Silsesquioxane to Functionalized Silicon Nanocrystals. Chem. Mater. 2017, 29, 80-89.

(14) Chiu, S. K.; Manhat, B. A.; De Benedetti, W. J. I.; Brown, A. L.; Fichter, K.; Vu, T.; Eastman, M.; Jiao, J.; Goforth, A. M. Aqueous Red-Emitting Silicon Nanoparticles for Cellular Imaging: Consequences of Protecting against Surface Passivation by Hydroxide and Water for Stable Red Emission. J. Mater. Res. 2013, 28, 216-230.

(15) Debenedetti, W. J. I.; Chiu, S. K.; Radlinger, C. M.; Ellison, R. J.; Manhat, B. A.; Zhang, J. Z.; Shi, J.; Goforth, A. M. Conversion from Red to Blue Photoluminescence in Alcohol Dispersions of Alkyl-Capped Silicon Nanoparticles: Insight into the Origins of Visible Photoluminescence in Colloidal Nanocrystalline Silicon. J. Phys. Chem. C 2015, 119, 9595-9608.

(16) Fuzell, J.; Thibert, A.; Atkins, T. M.; Dasog, M.; Busby, E.; Veinot, J. G. C.; Kauzlarich, S. M.; Larsen, D. S. Red States versus Blue States in Colloidal Silicon Nanocrystals: Exciton Sequestration into Low-Density Traps. J. Phys. Chem. Lett. 2013, 4, 3806-3812.

(17) Oliynyk, A. O.; Veinot, J. G. C.; Hooper, R. W.; Thiessen, A. N.; Ha, M.; Michaelis, V. K.; Yu, H. Silicon Nanoparticles: Are They Crystalline from the Core to the Surface? Chem. Mater. 2019, 31, 678-688.

(18) Bamiedakis, N.; Williams, K. A.; Penty, R. V; White, I. H. Chapter 11 Integrated and Hybrid Photonics for High-Performance Interconnects. In Optics and Photonics; Kaminow, I. P.; Li, T.; Willner, A. E. B. T.-O. F. T. (Sixth E., Eds.; Academic Press: Boston, 2013; pp. 377-418.

(19) Kanemitsu, Y.; Ogawa, T.; Shiraishi, K.; Takeda, K. Visible Photoluminescence from Oxidized Si Nanometer-Sized Spheres: Exciton Confinement on a Spherical Shell. Phys. Rev. B 1993, 48, 4883-4886.

(20) Wolkin, M. V.; Jorne, J.; Fauchet, P. M.; Allan, G.; Delerue, C. Electronic States and Luminescence in Porous Silicon Quantum Dots: The Role of Oxygen. Phys. Rev. Lett. 1999, 82, 197-200.

(21) Bawendi, M. G.; Steigerwald, M. L.; Brus, L. E. The Quantum Mechanics of Larger Semiconductor Clusters ("Quantum Dots"). Annu. Rev. Phys. Chem. 1990, 41, 477-496.

(22) Alivisatos, A. P. Perspectives on the Physical Chemistry of Semiconductor Nanocrystals. J. Phys. Chem. 1996, 100, 13226-13239.

(23) Trwoga, P. F.; Kenyon, A. J.; Pitt, C. W. Modeling the Contribution of Quantum Confinement to Luminescence from Silicon Nanoclusters. J. Appl. Phys. 1998, 83, 3789-3794.

(24) Barbagiovanni, E. G.; Lockwood, D. J.; Simpson, P. J.; Goncharova, L. V. Quantum Confinement in $\mathrm{Si}$ and Ge Nanostructures: Theory and Experiment. Appl. Phys. Rev. 2014, 1.

(25) Meier, C.; Gondorf, A.; Lüttjohann, S.; Lorke, A.; Wiggers, H. Silicon Nanoparticles: Absorption, Emission, and the Nature of the Electronic Bandgap. J. Appl. Phys. 2007, 101.

(26) Nayfeh, M. H.; Rigakis, N.; Yamani, Z. Photoexcitation of Si-Si Surface 
States in Nanocrystallites. Phys. Rev. B - Condens. Matter Mater. Phys. 1997, 56, 2079-2084.

(27) Pan, B. C.; Biswas, R. Structure and Simulation of Hydrogenated Nanocrystalline Silicon. J. Appl. Phys. 2004, 96, 6247-6252.

(28) Delerue, C.; Allan, G.; Lannoo, M. Theoretical Aspects of the Luminescence of Porous Silicon. Phys. Rev. B 1993, 48, 11024-11036.

(29) Dohnalová, K.; Gregorkiewicz, T.; Kůsová, K. Silicon Quantum Dots: Surface Matters. J. Phys. Condens. Matter 2014, 26.

(30) Kelly, J. a; Henderson, E. J.; Veinot, J. G. C. Wavelength-Controlled Etching of Silicon Nanocrystals. 2011.

(31) Ledoux, G.; Gong, J.; Huisken, F. Effect of Passivation and Aging on the Photoluminescence of Silicon Nanocrystals. Appl. Phys. Lett. 2001, 79, 4028-4030.

(32) Dasog, M.; Veinot, J. G. C. Tuning Silicon Quantum Dot Luminescence via Surface Groups. Phys. Status Solidi Basic Res. 2014, 251, 2216-2220.

(33) Buriak, J. M.; Stewart, M. P.; Geders, T. W.; Allen, M. J.; Choi, H. C.; Smith, J.; Raftery, D.; Canham, L. T. Lewis Acid Mediated Hydrosilylation on Porous Silicon Surfaces. J. Am. Chem. Soc. 1999, 121, 11491-11502.

(34) Cicero, R. L.; Linford, M. R.; Chidsey, C. E. D. Photoreactivity of Unsaturated Compounds with Hydrogen-Terminated Silicon(111). Langmuir 2000, 16, 5688-5695.

(35) Stewart, M. P.; Buriak, J. M. Exciton-Mediated Hydrosilylation on Photoluminescent Nanocrystalline Silicon. J. Am. Chem. Soc. 2001, 123, 7821-7830.

(36) Linford, M. R.; Chidsey, C. E. D.; Fenter, P.; Eisenberger, P. M. Alkyl Monolayers on Silicon Prepared from 1-Alkenes and Hydrogen-Terminated Silicon. J. Am. Chem. Soc. 1995, 117, 3145-3155.

(37) Boukherroub, R.; Morin, S.; Wayner, D. D. M.; Lockwood, D. J. Thermal Route for Chemical Modification and Photoluminescence Stabilization of Porous Silicon. Phys. Status Solidi Appl. Res. 2000, 182, 117-121.

(38) Boukherroub, R.; Morin, S.; Wayner, D. D. M.; Bensebaa, F.; Sproule, G. I.; Baribeau, J. M.; Lockwood, D. J. Ideal Passivation of Luminescent Porous Silicon by Thermal, Noncatalytic Reaction with Alkenes and Aldehydes. Chem. Mater. 2001, 13, 2002-2011.

(39) Clark, R. J.; Dang, M. K. M.; Veinot, J. G. C. Exploration of Organic Acid Chain Length on Water-Soluble Silicon Quantum Dot Surfaces. Langmuir 2010, 26, 15657-15664.

(40) Yang, Z.; Gonzalez, C. M.; Purkait, T. K.; lqbal, M.; Meldrum, A.; Veinot, J. G. C. Radical Initiated Hydrosilylation on Silicon Nanocrystal Surfaces: An Evaluation of Functional Group Tolerance and Mechanistic Study. Langmuir 2015, 31, 10540-10548.

(41) Lee, B. G.; Hiller, D.; Luo, J. W.; Semonin, O. E.; Beard, M. C.; Zacharias, M.; Stradins, P. Strained Interface Defects in Silicon Nanocrystals. Adv. Funct. Mater. 2012, 22, 3223-3232. 
(42) Kleovoulou, K.; Kelires, P. C. Stress State of Embedded Si Nanocrystals. Phys. Rev. B - Condens. Matter Mater. Phys. 2013, 88, 1-5.

(43) Shu, Y.; Kortshagen, U. R.; Levine, B. G.; Anthony, R. J. Surface Structure and Silicon Nanocrystal Photoluminescence: The Role of Hypervalent Silyl Groups. J. Phys. Chem. C 2015, 119, 26683-26691.

(44) Dasog, M.; Yang, Z.; Regli, S.; Atkins, T. M.; Faramus, A.; Singh, M. P.; Muthuswamy, E.; Kauzlarich, S. M.; Tilley, R. D.; Veinot, J. G. C. Chemical Insight into the Origin of Red and Blue Photoluminescence Arising from Freestanding Silicon Nanocrystals. ACS Nano 2013, 7, 2676-2685.

(45) Li, X.; He, Y.; Talukdar, S. S.; Swihart, M. T. Process for Preparing Macroscopic Quantities of Brightly Photoluminescent Silicon Nanoparticles with Emission Spanning the Visible Spectrum. Langmuir 2003, 19, 84908496.

(46) Zhang, X.; Neiner, D.; Wang, S.; Louie, A. Y.; Kauzlarich, S. M. A New Solution Route to Hydrogen-Terminated Silicon Nanoparticles: Synthesis, Functionalization and Water Stability. Nanotechnology 2007, 18.

(47) Manhat, B. A.; Brown, A. L.; Black, L. A.; Ross, J. B. A.; Fichter, K.; Vu, T.; Richman, E.; Goforth, A. M. One-Step Melt Synthesis of Water-Soluble, Photoluminescent, Surface-Oxidized Silicon Nanoparticles for Cellular Imaging Applications. Chem. Mater. 2011, 23, 2407-2418.

(48) Bley, R. A.; Kauzlarich, S. M. A Low-Temperature Solution Phase Route for the Synthesis of Silicon Nanoclusters. J. Am. Chem. Soc. 1996, 118, 12461-12462.

(49) Yang, C. S.; Bley, R. A.; Kauzlarich, S. M.; Lee, H. W. H.; Delgado, G. R. Synthesis of Alkyl-Terminated Silicon Nanoclusters by a Solution Route. J. Am. Chem. Soc. 1999, 121, 5191-5195.

(50) Du, X. W.; Qin, W. J.; Lu, Y. W.; Han, X.; Fu, Y. S.; Hu, S. L. FaceCentered-Cubic Si Nanocrystals Prepared by Microsecond Pulsed Laser Ablation. J. Appl. Phys. 2007, 102.

(51) Yang, S.; Li, W.; Cao, B.; Zeng, H.; Cai, W. Origin of Blue Emission from Silicon Nanoparticles: Direct Transition and Interface Recombination. J. Phys. Chem. C 2011, 115, 21056-21062.

(52) Hessel, C. M.; Henderson, E. J.; Veinot, J. G. C. Hydrogen Silsesquioxane: A Molecular Precursor for Nanocrystalline Si-SiO2 Composites and Freestanding Hydride-Surface-Terminated Silicon Nanoparticles. Chem. Mater. 2006, 18, 6139-6146.

(53) Hessel, C. M.; Reid, D.; Panthani, M. G.; Rasch, M. R.; Goodfellow, B. W.; Wei, J.; Fujii, H.; Akhavan, V.; Korgel, B. A. Synthesis of Ligand-Stabilized Silicon Nanocrystals with Size-Dependent Photoluminescence Spanning Visible to near-Infrared Wavelengths. Chem. Mater. 2012, 24, 393-401.

(54) Wilcoxon, J. P.; Samara, G. A.; Provencio, P. N. Optical and Electronic Properties of Si Nanoclusters Synthesized in Inverse Micelles. Phys. Rev. B - Condens. Matter Mater. Phys. 1999, 60, 2704-2714. Warner, J. H.; Rubinsztein-Dunlop, H.; Tilley, R. D. Surface Morphology 
Dependent Photoluminescence from Colloidal Silicon Nanocrystals. J. Phys. Chem. B 2005, 109, 19064-19067.

(56) Kislitsyn, D. A.; Mills, J. M.; Chiu, S. K.; Taber, B. N.; Barnes, J. D.; Gervasi, C. F.; Goforth, A. M.; Nazin, G. V. Creation and Annihilation of Charge Traps in Silicon Nanocrystals: Experimental Visualization and Spectroscopy. J. Phys. Chem. Lett. 2018, 9, 710-716.

(57) Chabal, Y. J.; Higashi, G. S.; Raghavachari, K.; Burrows, V. A. Infrared Spectroscopy of $\mathrm{Si}(111)$ and $\mathrm{Si}(100)$ Surfaces after HF Treatment: Hydrogen Termination and Surface Morphology. J. Vac. Sci. Technol. A 1989, 7, 2104-2109.

(58) Trucks, G. W.; Raghavachari, K.; Higashi, G. S.; Chabal, Y. J. Mechanism of HF Etching of Silicon Surfaces: A Theoretical Understanding of Hydrogen Passivation. Phys. Rev. Lett. 1990, 65, 504-507.

(59) Huisken, F.; Ledoux, G.; Guillois, O.; Reynaud, C. Light-Emitting Silicon Nanocrystals from Laser Pyrolysis. Adv. Mater. 2002, 14, 1861-1865.

(60) Radlinger, C. M. Investigation into Effects of Instability and Reactivity of Hydride-Passivated Silicon Nanoparticles on Interband Photoluminescence, Portland State University, 2017.

(61) Buriak, J. M.; Stewart, M. P.; Geders, T. W.; Allen, M. J.; Choi, H. C.; Smith, J.; Raftery, D.; Canham, L. T. Lewis Acid Mediated Hydrosilylation on Porous Silicon Surfaces. J. Am. Chem. Soc. 1999, 121, 11491-11502.

(62) Henderson, E. J.; Kelly, J. A.; Veinot, J. G. C. Influence of HSiO ${ }_{1.5}$ Sol-Gel Polymer Structure and Composition on the Size and Luminescent Properties of Silicon Nanocrystals. Chem. Mater. 2009, 21, 5426-5434.

(63) He, J. W.; Xu, X.; Corneille, J. S.; Goodman, D. W. X-Ray Photoelectron Spectroscopic Characterization of Ultra-Thin Silicon Oxide Films on a Mo(100) Surface. Surf. Sci. 1992, 279, 119-126.

(64) Zhou, T.; Anderson, R. T.; Li, H.; Bell, J.; Yang, Y.; Gorman, B. P.; Pylypenko, S.; Lusk, M. T.; Sellinger, A. Bandgap Tuning of Silicon Quantum Dots by Surface Functionalization with Conjugated Organic Groups. Nano Lett. 2015, 15, 3657-3663.

(65) Wei, W.; Xu, G.; Wang, J.; Wang, T. Raman Spectra of Intrinsic and Doped Hydrogenated Nanocrystalline Silicon Films. Vacuum 2007, 81, 656-662.

(66) Rosso-Vasic, M.; Spruijt, E.; Van Lagen, B.; De Cola, L.; Zuilhof, H. AlkylFunctionalized Oxide-Free Silicon Nanoparticles: Synthesis and Optical Properties. Small 2008, 4, 1835-1841.

(67) Yang, C. C.; Chen, W. C. The Structures and Properties of Hydrogen Silsesquioxane (Hsq) Films Produced by Thermal Curing. J. Mater. Chem. 2002, 12, 1138-1141.

(68) Launer, P. J. Infrared Analysis of Organosilicon Compounds: SpectraStructure Correlations. Silicone Compd. Regist. Rev. 1987, 100-103.

(69) Loboda, M. J.; Grove, C. M.; Schneider, R. F. Properties of a - SiO x : H Thin Films Deposited from Hydrogen Silsesquioxane Resins. J. Electrochem. Soc. 1998, 145, 2861-2866. 
(70) Kovalev, D.; Gross, E.; Diener, J.; Yu Timoshenko, V.; Fujii, M. Photodegradation of Porous Silicon Induced by Photogenerated Singlet Oxygen Molecules. Appl. Phys. Lett. 2004, 85, 3590-3592.

(71) Lapkin, A. A.; Boddu, V. M.; Aliev, G. N.; Goller, B.; Polisski, S.; Kovalev, D. Photo-Oxidation by Singlet Oxygen Generated on Nanoporous Silicon in a LED-Powered Reactor. Chem. Eng. J. 2008, 136, 331-336.

(72) Song, J. H.; Sailor, M. J. Dimethyl Sulfoxide as a Mild Oxidizing Agent for Porous Silicon and Its Effect on Photoluminescence. Inorg. Chem. 1998, 37, 3355-3360.

(73) Ledoux, G.; Guillois, O.; Porterat, D.; Reynaud, C.; Huisken, F.; Kohn, B.; Paillard, V. Photoluminescence Properties of Silicon Nanocrystals as a Function of Their Size. Phys. Rev. B 2000, 62, 15942-15951.

(74) Hua, F.; Erogbogbo, F.; Swihart, M. T.; Ruckenstein, E. Organically Capped Silicon Nanoparticles with Blue Photoluminescence Prepared by Hydrosilylation Followed by Oxidation. Langmuir 2006, 22, 4363-4370.

(75) Gupta, A.; Swihart, M. T.; Wiggers, H. Luminescent Colloidal Dispersion of Silicon Quantum Dots from Microwave Plasma Synthesis: Exploring the Photoluminescence Behavior across the Visible Spectrum. Adv. Funct. Mater. 2009, 19, 696-703.

(76) Winters, B. J.; Holm, J.; Roberts, J. T. Thermal Processing and Native Oxidation of Silicon Nanoparticles. J. Nanoparticle Res. 2011, 13, 54735484.

(77) Dasog, M.; De Los Reyes, G. B.; Titova, L. V.; Hegmann, F. A.; Veinot, J. G. C. Size vs Surface: Tuning the Photoluminescence of Freestanding Silicon Nanocrystals Across the Visible Spectrum via Surface Groups. ACS Nano 2014, 8, 9636-9648.

(78) Yang, C.-S.; Kauzlarich, S. M.; Wang, Y. C.; Lee, H. W. H. Photoluminescence as a Function of Aggregated Size from N-ButylTerminated Silicon Nanoclusters. J. Clust. Sci. 2000, 11, 423-431.

(79) Ghosh, B.; Shirahata, N. Colloidal Silicon Quantum Dots: Synthesis and Luminescence Tuning from the near-UV to the near-IR Range. Sci. Technol. Adv. Mater. 2014, 15.

(80) Li, S.; Silvers, S. J.; El-Shall, M. S. Surface Oxidation and Luminescence Properties of Weblike Agglomeration of Silicon Nanocrystals Produced by a Laser Vaporization-Controlled Condensation Technique. J. Phys. Chem. B 1997, 101, 1794-1802.

(81) Zhou, Z.; Brus, L.; Friesner, R. Electronic Structure and Luminescence of 1.1- and 1.4-Nm Silicon Nanocrystals: Oxide Shell versus Hydrogen Passivation. Nano Lett. 2003, 3, 163-167.

(82) Švrček, V.; Rehspringer, J. L.; Gaffet, E.; Slaoui, A.; Muller, J. C. Unaggregated Silicon Nanocrystals Obtained by Ball Milling. J. Cryst. Growth 2005, 275, 589-597.

(83) Lam, C.; Zhang, Y. F.; Tang, Y. H.; Lee, C. S.; Bello, I.; Lee, S. T. LargeScale Synthesis of Ultrafine Si Nanoparticles by Ball Milling. J. Cryst. 
Growth 2000, 220, 466-470.

(84) Bao, Z.; Weatherspoon, M. R.; Shian, S.; Cai, Y.; Graham, P. D.; Allan, S. M.; Ahmad, G.; Dickerson, M. B.; Church, B. C.; Kang, Z.; et al. Chemical Reduction of Three-Dimensional Silica Micro-Assemblies into Microporous Silicon Replicas. Nature 2007, 446, 172-175.

(85) Guo, M.; Zou, X.; Ren, H.; Muhammad, F.; Huang, C.; Qiu, S.; Zhu, G. Fabrication of High Surface Area Mesoporous Silicon via Magnesiothermic Reduction for Drug Delivery. Microporous Mesoporous Mater. 2011, 142, 194-201.

(86) Xie, J.; Wang, G.; Huo, Y.; Zhang, S.; Cao, G.; Zhao, X. Nanostructured Silicon Spheres Prepared by a Controllable Magnesiothermic Reduction as Anode for Lithium Ion Batteries. Electrochim. Acta 2014, 135, 94-100. Liu, X.; Gao, Y.; Jin, R.; Luo, H.; Peng, P.; Liu, Y. Scalable Synthesis of Si Nanostructures by Low-Temperature Magnesiothermic Reduction of Silica for Application in Lithium Ion Batteries. Nano Energy 2014, 4, 31-38.

(88) Kim, K. H.; Lee, D. J.; Cho, K. M.; Kim, S. J.; Park, J. K.; Jung, H. T. Complete Magnesiothermic Reduction Reaction of Vertically Aligned Mesoporous Silica Channels to Form Pure Silicon Nanoparticles. Sci. Rep. 2015, 5, 1-7.

(89) Favors, Z.; Wang, W.; Bay, H. H.; Mutlu, Z.; Ahmed, K.; Liu, C.; Ozkan, M.; Ozkan, C. S. Scalable Synthesis of Nano-Silicon from Beach Sand for Long Cycle Life Li-lon Batteries. Sci. Rep. 2014, 4, 1-7.

(90) Dorval Courchesne, N. M.; Steiner, S. A.; Cantú, V. J.; Hammond, P. T.; Belcher, A. M. Biotemplated Silica and Silicon Materials as Building Blocks for Micro- to Nanostructures. Chem. Mater. 2015, 27, 5361-5370.

(91) Lai, Y.; Thompson, J. R.; Dasog, M. Metallothermic Reduction of Silica Nanoparticles to Porous Silicon for Drug Delivery Using New and Existing Reductants. Chem. - A Eur. J. 2018, 24, 7913-7920.

(92) Barati, M.; Sarder, S.; McLean, A.; Roy, R. Recovery of Silicon from Silica Fume. J. Non. Cryst. Solids 2011, 357, 18-23.

(93) Xing, A.; Zhang, J.; Bao, Z.; Mei, Y.; Gordin, A. S.; Sandhage, K. H. A Magnesiothermic Reaction Process for the Scalable Production of Mesoporous Silicon for Rechargeable Lithium Batteries. Chem. Commun. 2013, 49, 6743.

(94) Liu, N.; Huo, K.; McDowell, M. T.; Zhao, J.; Cui, Y. Rice Husks as a Sustainable Source of Nanostructured Silicon for High Performance Li-lon Battery Anodes. Sci. Rep. 2013, 3, 1-7.

(95) Balzar, D. X-Ray Diffraction Line Broadening: Modeling and Applications to High-Tc Superconductors. J. Res. Natl. Inst. Stand. Technol. 1993, 98, 321.

(96) Dasog, M.; Yang, Z.; Veinot, J. G. C. Size-Controlled Solid State Synthesis of Luminescent Silicon Nanocrystals Using Stöber Silica Particles. CrystEngComm 2012, 14, 7576. 